\title{
MODELING AND CHARGING CONTROL OF A \\ LITHIUM ION BATTERY SYSTEM \\ FOR SOLAR PANELS
}

\author{
A Thesis \\ presented to \\ the Faculty of California Polytechnic State University, \\ San Luis Obispo
}

\author{
In Partial Fulfillment \\ of the Requirements for the Degree \\ Master of Science in Electrical Engineering
}

by

Garrett David Heinen

June 2017 
(C) 2017

Garrett David Heinen

\section{ALL RIGHTS RESERVED}




\section{COMMITTEE MEMBERSHIP}

TITLE: Modeling and Charging Control of a

Lithium Ion Battery System for Solar Panels

AUTHOR: Garrett David Heinen

DATE SUBMITTED: June 2017

COMMITTEE CHAIR: Xiao-Hua Yu, Ph.D.

Professor of Electrical Engineering

COMMITTEE MEMBER: Taufik, Ph.D.

Professor of Electrical Engineering

COMMITTEE MEMBER: $\quad$ Ahmad Nafisi, Ph.D.

Professor of Electrical Engineering 


\begin{abstract}
Modeling and Charging Control of a Lithium Ion Battery System for Solar Panels

Garrett David Heinen
\end{abstract}

The advancement in solar panel and battery technology makes them useful for energy supply and storage. This thesis involves the modeling and charging control of a lithium ion battery system for solar panels. The proposed model is based on the parameters and characteristics of a realistic battery and solar panel system; and the hybrid control approach combines the advantages of the adaptive incremental conductance method and the perturb and observe method to track the maximum power point of the solar panel for charging the battery unit. Computer simulation results demonstrate that this proposed approach offers a faster convergence rate than the adaptive incremental conductance method, and less steady-state error than the perturb and observe method.

Keywords: solar panel, power converter, Lithium Ion ( $\mathrm{Li}+)$, constant power to constant voltage (CP/CV), Maximum Power Point Tracking (MPPT), Perturb and Observe (P\&O), incremental conductance 


\section{TABLE OF CONTENTS}

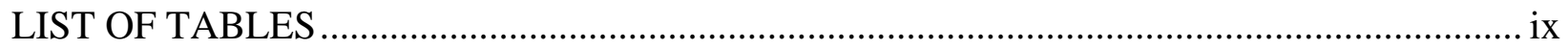

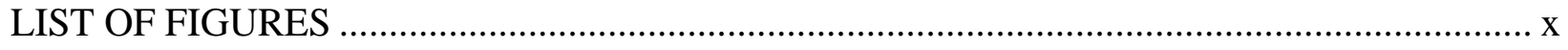

\section{CHAPTER}

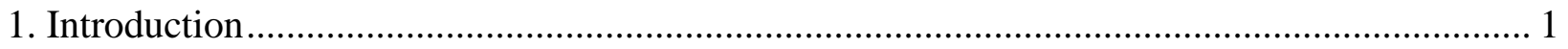

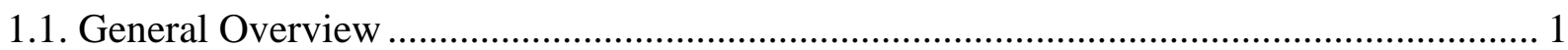

1.2. The Technical Problem ......................................................................................... 3

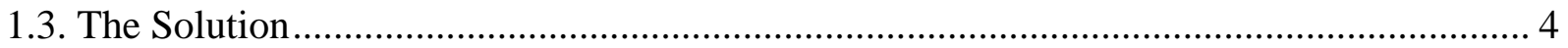

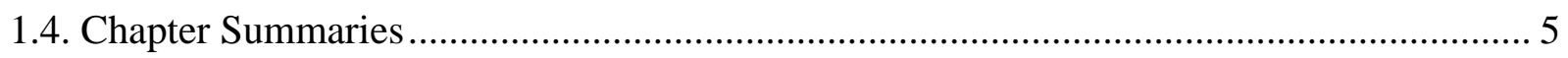

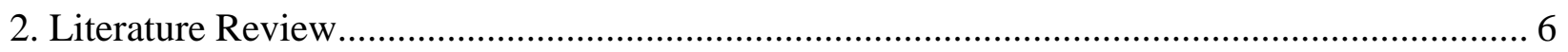

2.1. Solar Panel to Battery Chargers ......................................................................... 6

2.1.1. P\&O MPPT and CC/CV Charging for Lead-acid Battery ..................................... 6

2.1.2. Hill-climbing MPPT and CP/CV Charging for Li+ Battery .................................. 7

2.1.3. Incremental Conductance MPPT and Three Charge Methods for Lead-acid Battery... 7

2.1.4. P\&O MPPT and Charging for Lead-acid Battery ........................................... 7

2.2. Solar Panel Maximum Power Point Tracking Algorithms .......................................... 7

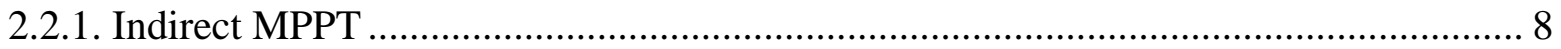

2.2.1.1. Fixed Voltage ......................................................................................... 8

2.2.1.2. Fractional Open Circuit Voltage ............................................................ 9

2.2.2. Direct MPPT with Physical Criteria.................................................................. 10

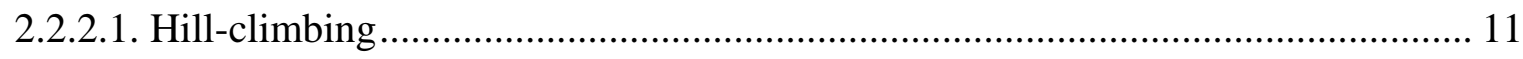

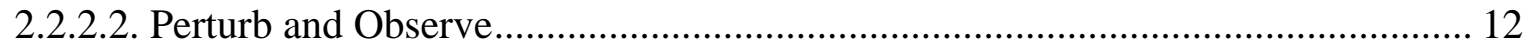


2.2.2.3. Incremental Conductance................................................................................ 14

2.2.2.4. Voltage/Current Sweep ……………………….......................................... 15

2.2.3. Direct MPPT with Abstract Criteria..................................................................... 16

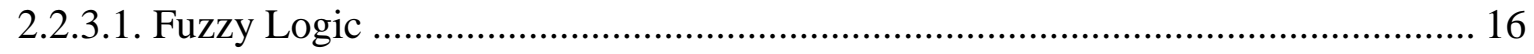

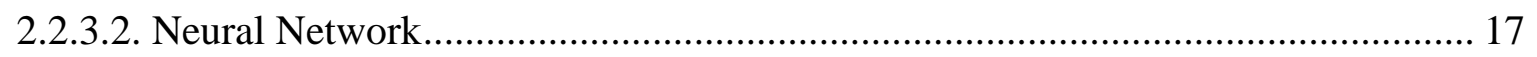

2.3. Lithium Ion Battery Charging Algorithms ................................................................. 19

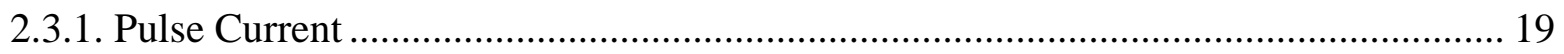

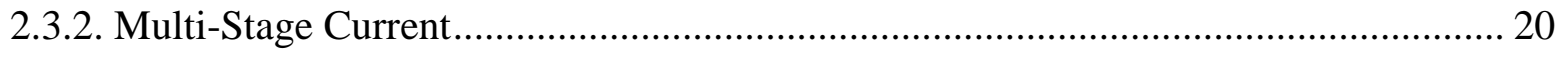

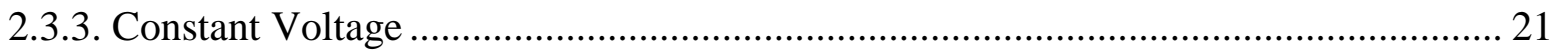

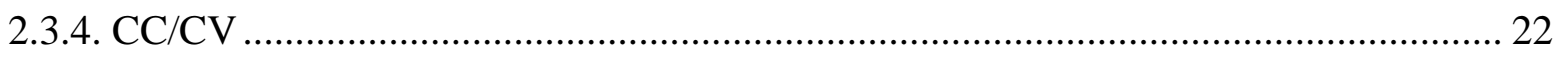

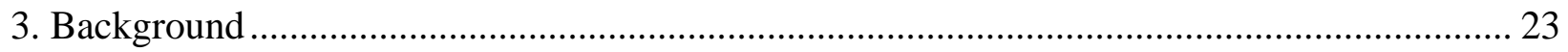

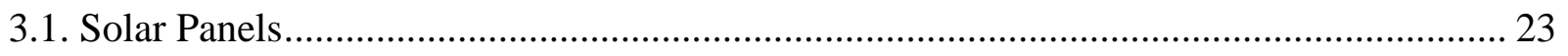

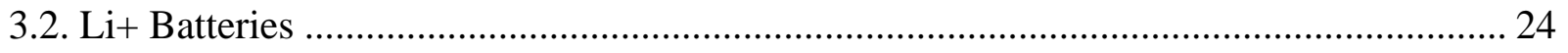

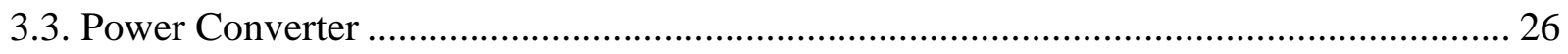

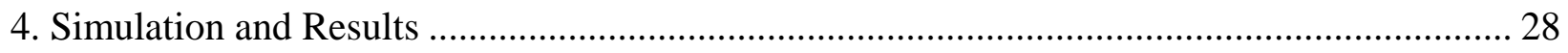

4.1. The Proposed Approach............................................................................................... 28

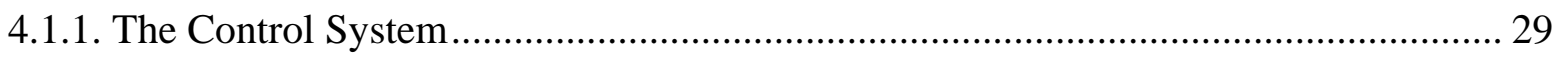

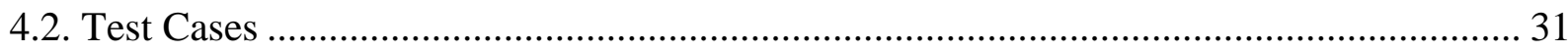

4.2.1. MPPT Control Test Case …………………......................................................... 32

4.2.2. Battery Voltage Control Test Case ........................................................................ 33

4.2.3. Battery Current Control Test Case ……………............................................................ 33

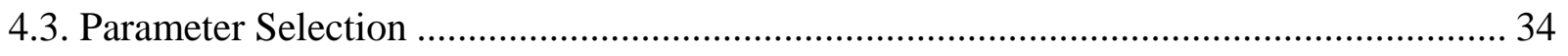

4.3.1. MPPT Control Parameters........................................................................................ 34 
4.3.2. Battery Voltage Control Parameters............................................................. 35

4.3.3. Battery Current Control Parameters ..................................................................... 36

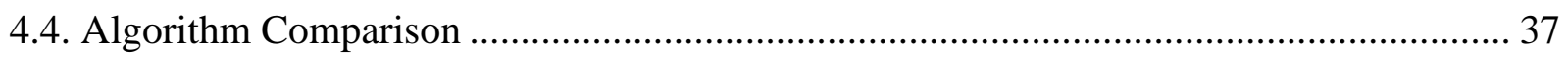

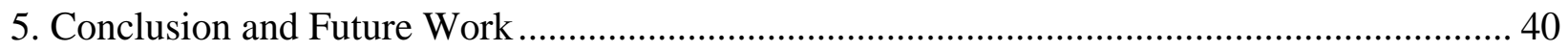

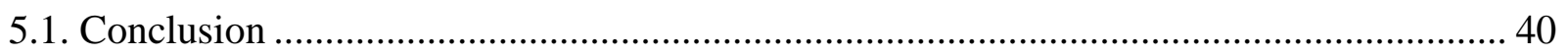

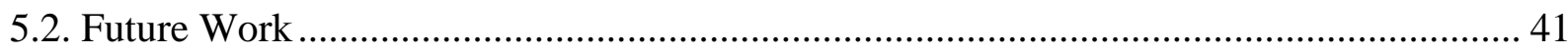

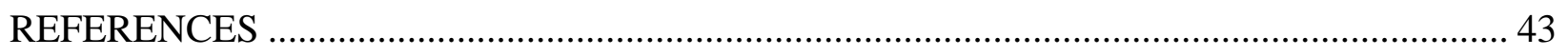

\section{APPENDICES}

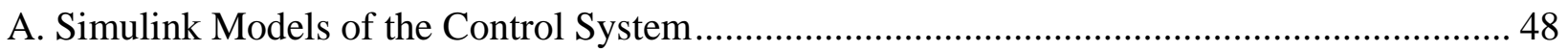

A.1. Simulink Model of Entire System.................................................................. 48

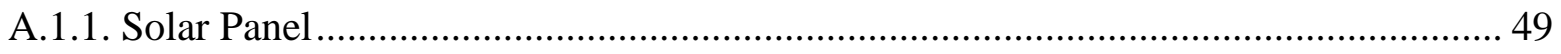

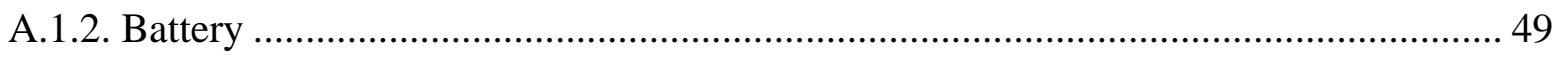

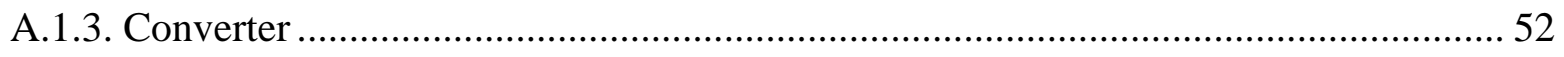

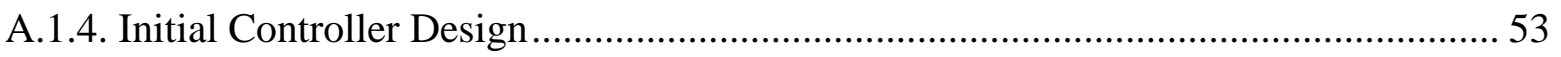

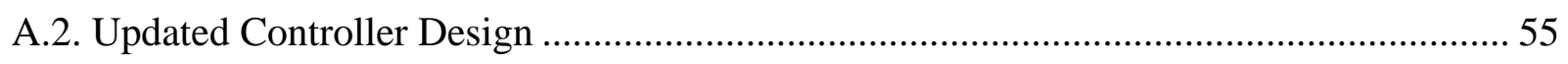

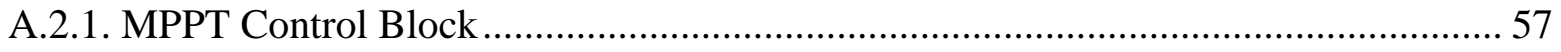

A.2.2. Battery Limiting Control Blocks ................................................................ 59

A.2.2.1. Battery Voltage Control Block ................................................................ 60

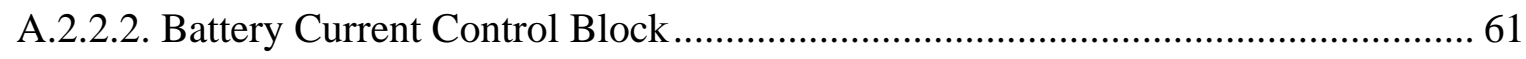

A.2.3. Duty Cycle Selection and Duty Cycle Separation Blocks................................... 62

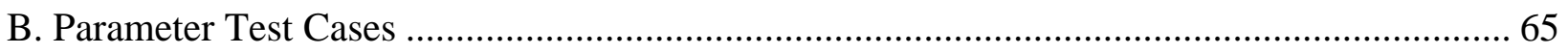

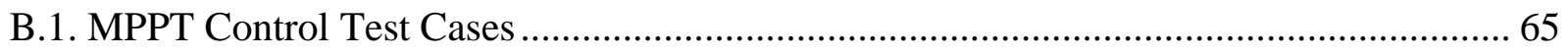

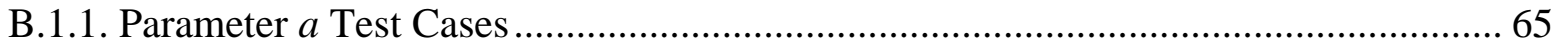




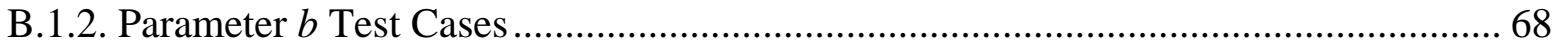

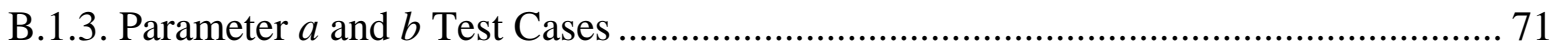

B.2. Battery Voltage Control Test Cases ............................................................................. 74

B.2.1. Parameter $c$ Test Cases ............................................................................................... 74

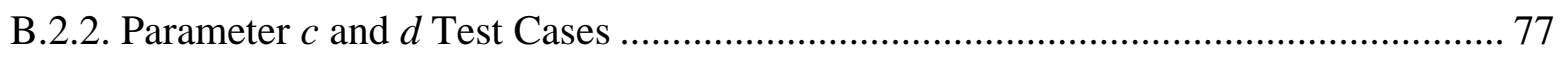

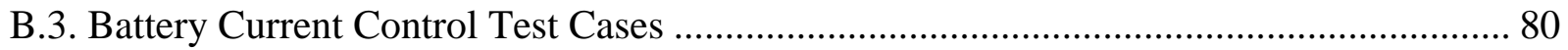

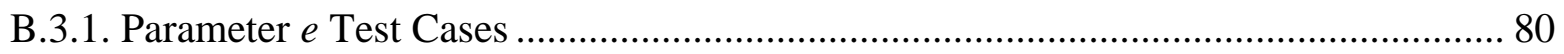

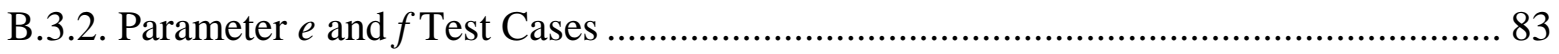




\section{LIST OF TABLES}

Page

Table 1. Performance Results of MPPT Methods ……….......................................................... 38 


\section{LIST OF FIGURES}

Page

Figure 1. Highest Level Block Diagram of Control System............................................... 4

Figure 2. Representation of MPP for a Solar Panel [5] f................................................ 8

Figure 3. PV system for use of Fractional Open Circuit Voltage [1] .................................... 10

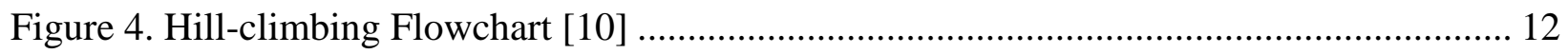

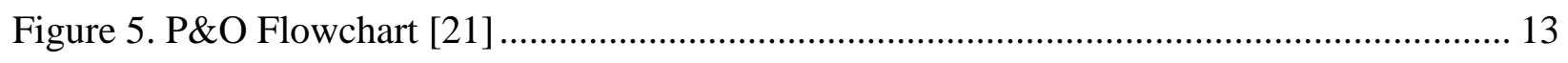

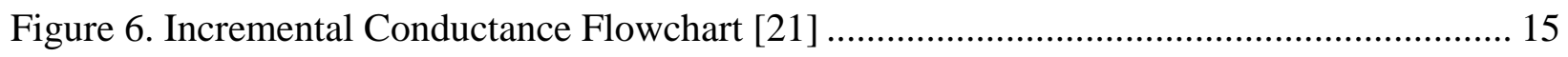

Figure 7. Example of Typical Membership Functions [5] ............................................... 17

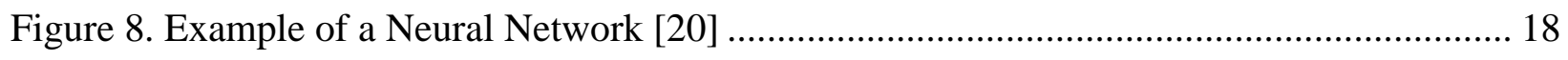

Figure 9. Pulse Current Charge [18] ........................................................................... 20

Figure 10. Multi-Stage Current Profile [18] .................................................................... 21

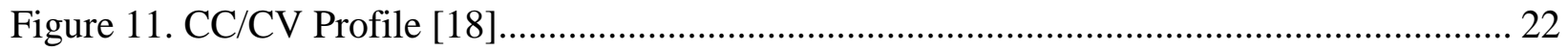

Figure 12. Current vs. Voltage with Varying Irradiance ................................................. 23

Figure 13. Equivalent Circuit of a Li+ Battery ............................................................ 25

Figure 14. Parameters of Li+ Battery Equivalent Circuit .............................................. 26

Figure 15. Model of a Non-Inverting Buck-Boost Converter............................................. 27

Figure 16. Block Diagram for Delta Duty Cycle ................................................................ 29

Figure 17. Block Diagram for Delta Duty Cycle MPPT …................................................ 30

Figure 18. Block Diagram for Delta Duty Cycle Critical ................................................. 31

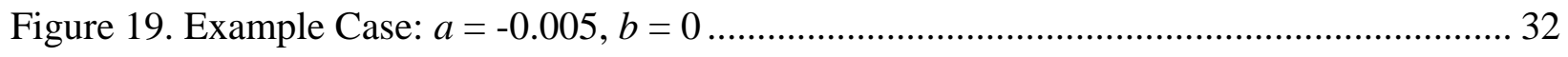

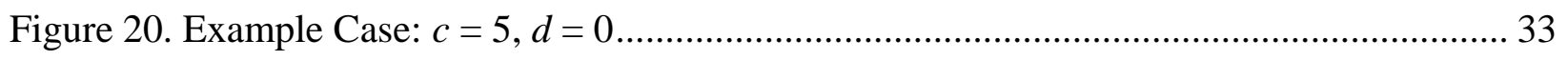

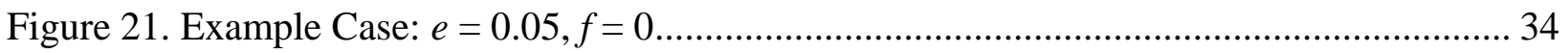




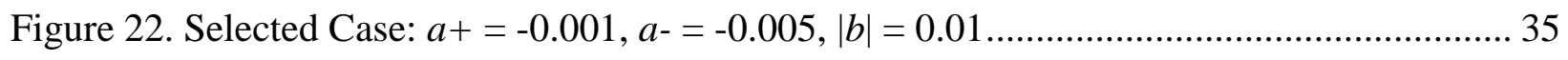

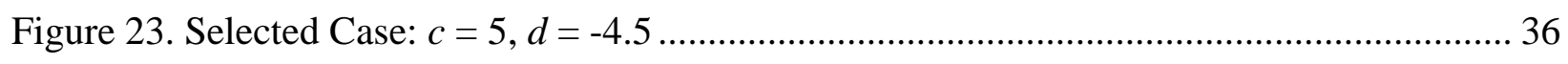

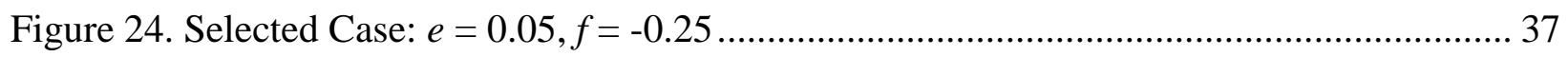

Figure 25. Comparison Plot for the Three Compared Methods................................................... 39

Figure 26. Example of Control System MPPT and Voltage Limiting ......................................... 41

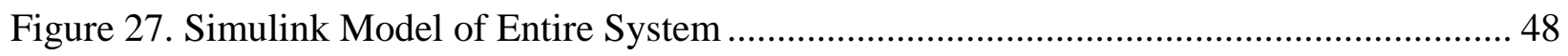

Figure 28. Simulink Model of Solar Panel .............................................................................. 49

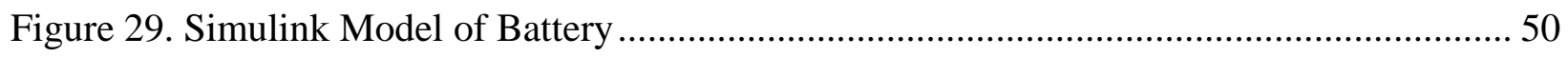

Figure 30. Simulink Model of the Li+ Battery Circuit ................................................................ 51

Figure 31. Simulink Model of RC Parallel Circuit ................................................................... 52

Figure 32. Simulink Model of Power Converter ................................................................ 53

Figure 33. Preliminary Simulink Model of Control System......................................................... 53

Figure 34. MATLAB Sample Code of Control Algorithm......................................................... 54

Figure 35. High Level Simulink Model of Control System.......................................................... 55

Figure 36. Low Level Simulink Model of Control System ......................................................... 56

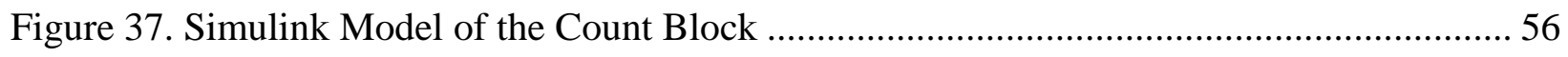

Figure 38. High Level Simulink Model of Duty Step - MPPT.................................................... 57

Figure 39. Simulink Model of MPPT Case Selection............................................................... 58

Figure 40. Simulink Model of MPPT Duty Step Selection ..................................................... 59

Figure 41. High Level Simulink Model of Duty Step - Critical ................................................... 60

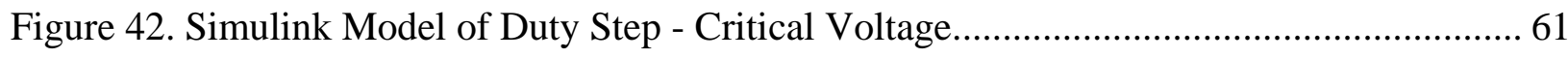

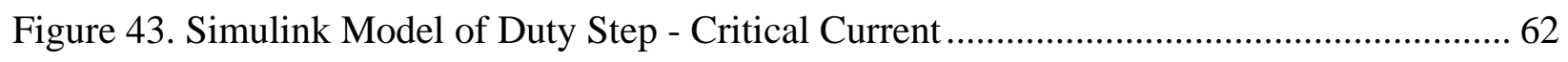

Figure 44. Simulink Model of Critical Duty Step Selection........................................................... 62 


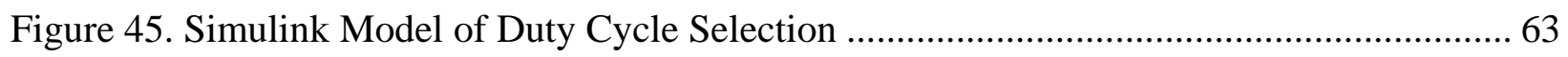

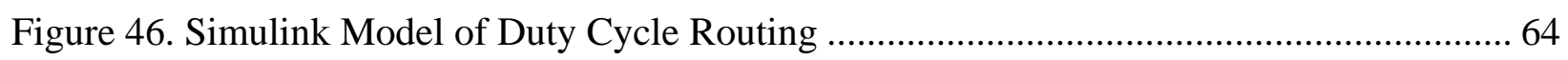

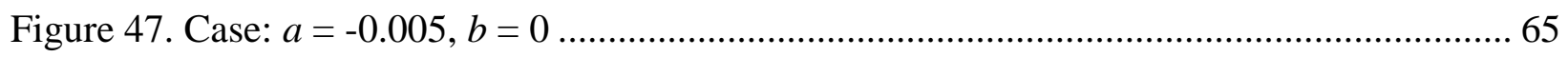

Figure 48. Case: $a=-0.01, b=0$

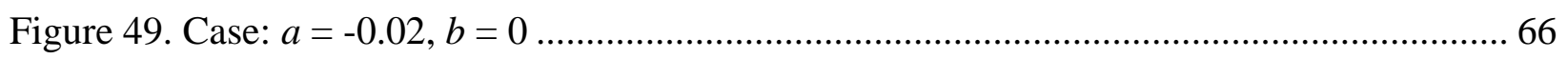

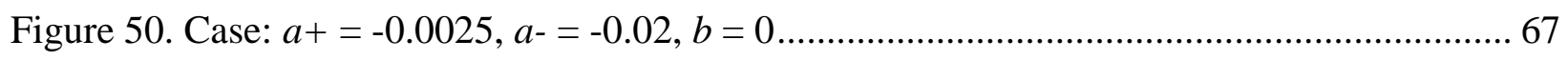

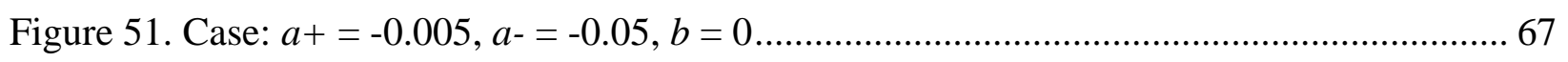

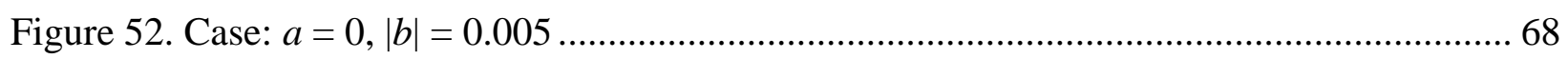

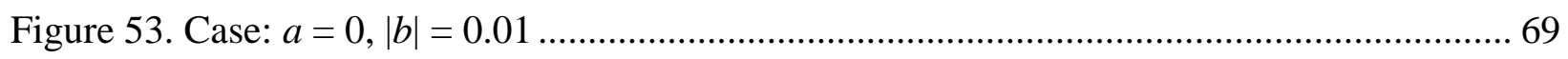

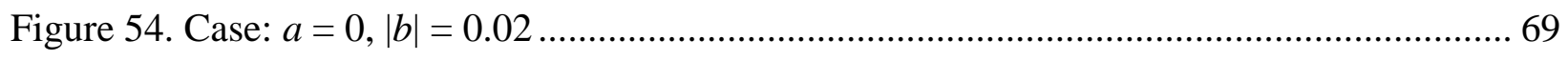

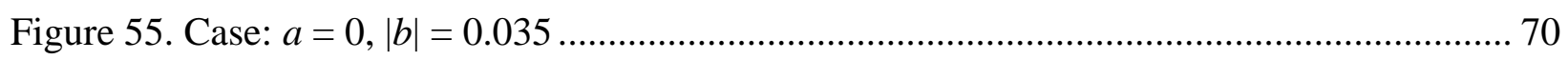

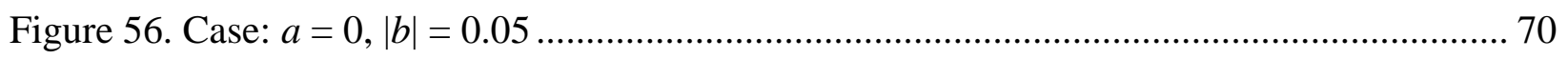

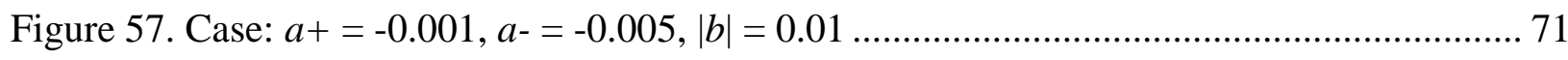

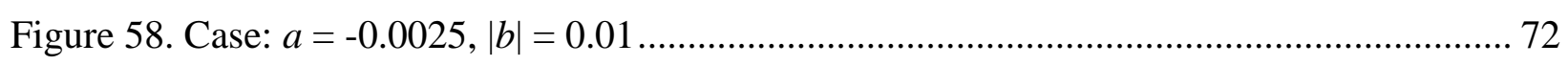

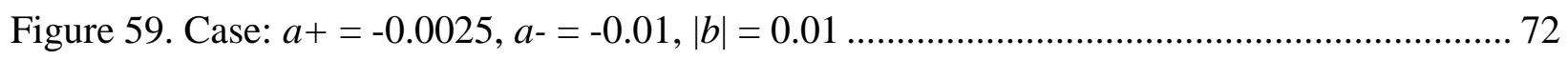

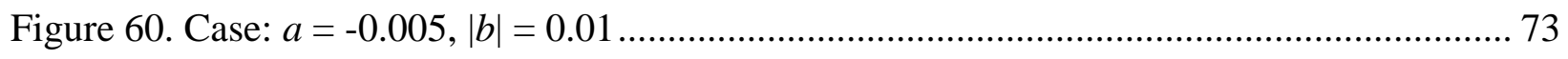

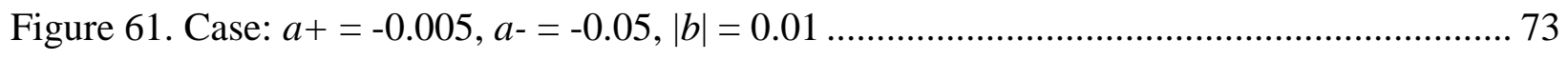

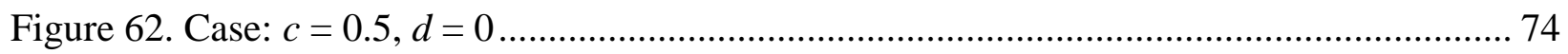

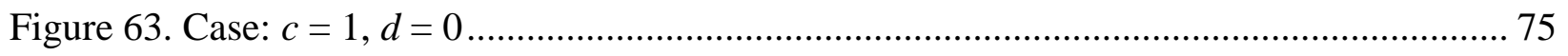

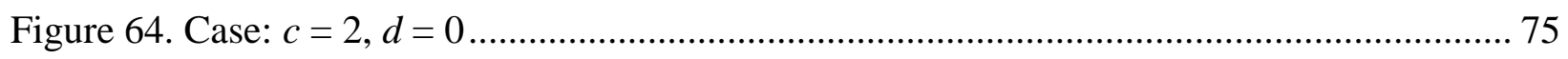

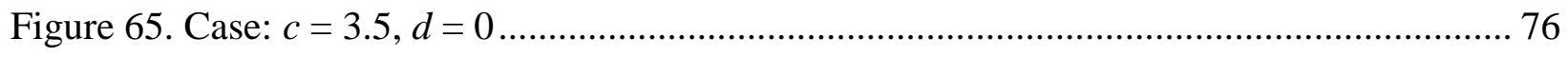

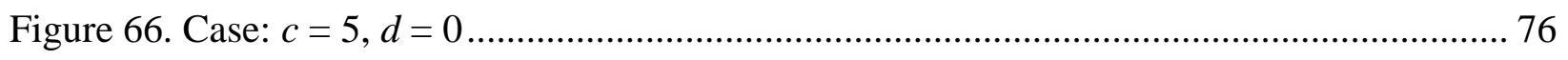

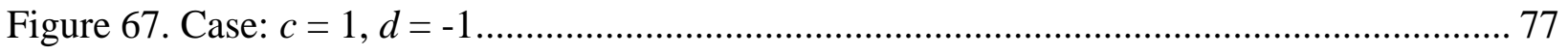




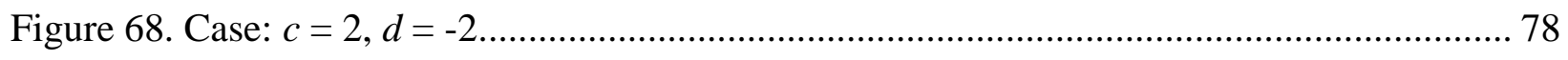

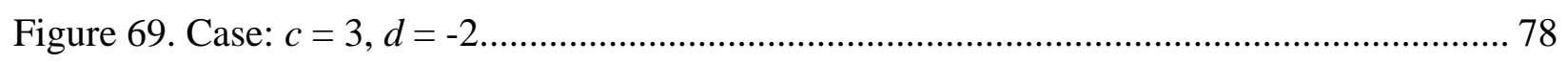

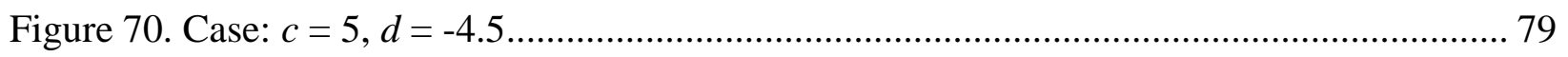

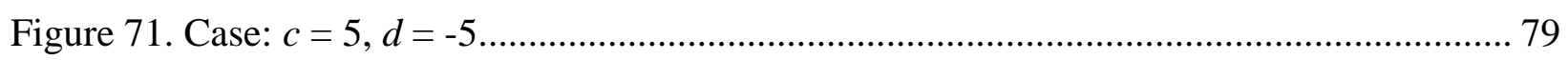

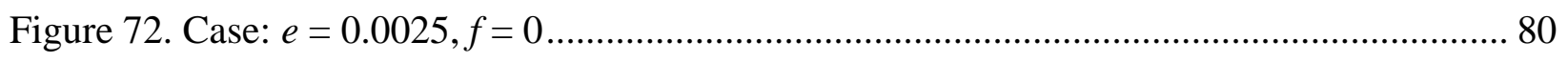

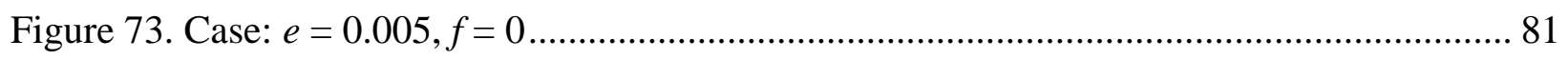

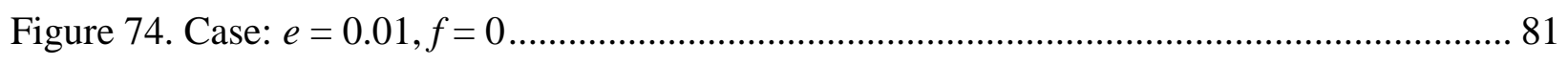

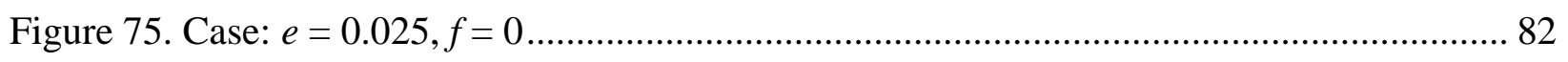

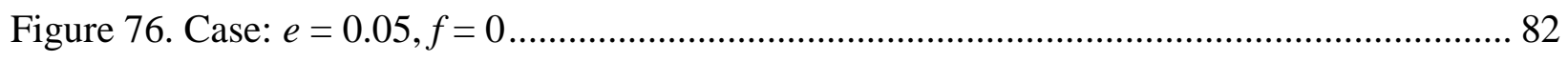

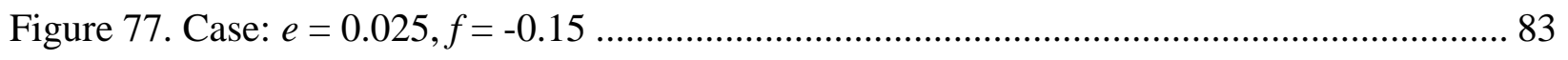

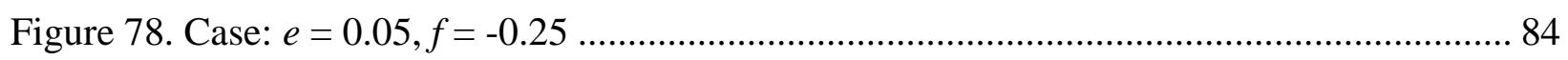

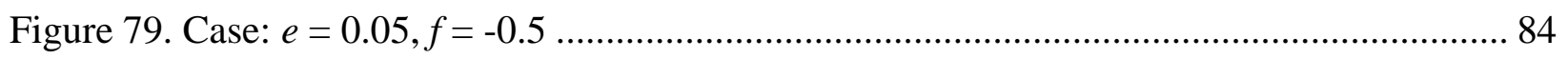

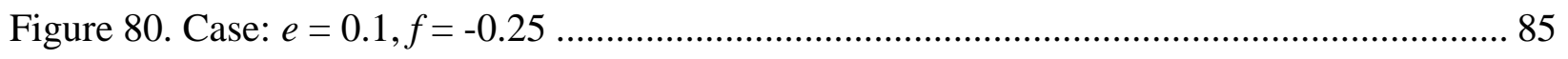

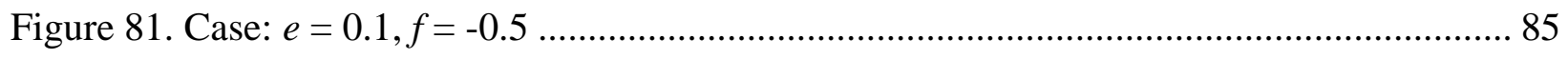




\section{Chapter 1. Introduction}

In this chapter, the advancements from gasoline to solar panels and batteries are explored, the technical problem is stated, the solution is discussed, and the other chapters are summarized.

\subsection{General Overview}

Every day more than $1 / 3$ of a billion gallons of gasoline is consumed inside the United States. Consuming gasoline at such a high rate has an extreme impact on the environment. If the gas is burned, which most of it is, it creates green-house gases into the atmosphere which absorbs the suns infrared radiation (heat) and results with an increase in temperature on Earth; this is also known as global warming.

The gasoline combustion reaction looks fairly clean and harmless since it produces $\mathrm{CO}_{2}$ and $\mathrm{H}_{2} \mathrm{O}$; however, $\mathrm{CO}_{2}$ and $\mathrm{H}_{2} \mathrm{O}$ are the largest contributors to the green-house effect, primarily $\mathrm{H}_{2} \mathrm{O}$. On top of this, in many cars and engines, the reactions are being contained inside small compartments with non-pure substances; therefore, higher damaging products get formed from the intense pressure and heat. Considering that the oxygen makes up $21 \%$ of the air and nitrogen is $78 \%$ of it, more nitrogen is inside the engine than there is oxygen. Because there is more than two times the amount of nitrogen than oxygen, the intense temperatures of the gasoline combustion combine there elements and create $\mathrm{NO}_{\mathrm{x}}$, which is critically worse than $\mathrm{CO}_{2}$. These green-house gases all have their level of damage, but $\mathrm{CO}_{2}$ is the highest amount produced.

Getting away from burning gasoline soon requires a significant jump into renewable energy sources such as solar, wind, or hydro. This energy is better being stored, preferably, in a re-usable storage such as a battery. Solar and battery technology has improved drastically over the most recent decade in the technological evolution. The prime reason for such a movement 
toward battery and solar growth is the need for environmentally safe technology. Solar cells are commercially available with rates at nearly $24.1 \%$ efficiency and have been researched up to $44.7 \%$ because of multi-level layer cell discoveries. With these efficiency levels, solar can now play a vital role on electric vehicles (EVs), specifically electric cars.

The electric car is capable of storing the energy onto its battery pack for present use or future use. However, the battery on the car is usually much larger than the battery for a house; but the house may have a larger solar system than the car does. Thankfully, batteries are currently being doped with different elements to enhance their properties towards certain applications, such as high power output capabilities for electric cars or high energy densities for houses. Unlike using coal or gasoline to run a house, there's an opportunity to utilize the roof for solar arrays and another location for a battery bank; it goes the same exact way for automobiles as well. Using solar power on a car is nice, especially when the car can't be readily hooked up to the grid, whereas, the sun power may be. It can save enough energy for some miles, electrical entertainment, or cooling/warming the battery packs. Operating both systems near the same voltage maximizes energy transfer and minimizes the amount of converting components between the two. Because the systems are likely to operate near the same voltage, their likely higher and lower values from each other, so a buck-boost converter is essential for proper energy transfer.

Introducing solar to electric cars is a revolutionary aspect to vehicles. There have been numerous production cars that operate simple devices on their solar panel power: Fisker's LeadAcid Battery, Prius' Fan, and a few others currently. However, the solar power from an EV can do more than just powering a fan or a couple appliances on board; it can charge the EV's battery pack with the new high solar efficiencies seen today. Storing the electrical energy in the battery pack is much more beneficial because it allows full operation of the vehicle. This is pure green 
energy that is harnessed directly from the sun and stored for the owner's instantaneous use. Solar is the ultimate energy source to use on an EV because there is no worry about hooking up to the grid where there might be coal produced energy. The final way to make solar officially greener then gas is to create the solar panels from solar panels; thus, there power needs to be stored.

\subsection{The Technical Problem}

As solar panels and batteries are immensely more powerful and important than before, integrating them together for power supplying is essential for the next century. Li+ batteries have greatly surpassed the uses of lead-acid batteries, and therefore are of higher importance to the overall system at study. The implementation of both solar panels and batteries on electric vehicles are useful due to these advancements. Thus, the technical problem being faced in this thesis is modeling a control system for an electric vehicle that enables maximum power transfer from its solar panel to its battery without harming the battery's health. Modeling and simulating control systems reveal potential issues prior to their onboard experimentation. Specifically designed control systems significantly boost the efficacy and overall use of the power, so more energy is harvested and utilized, and less energy is wasted and given off as pollution to the environment.

The algorithm for the control system needs to have higher analyzing capability then other algorithms currently implemented to improve the quality of the entire system. The algorithm is robust and doesn't fail to constantly track the necessary power point. When the EV is being driven, the voltage to operate the panel changes because the irradiance of light on the panel is changing with the angle from the sun light; thus, the solar panels current to voltage curve changes, so the algorithm tracks this MPP change. The algorithm also charges lithium ion 
batteries, which aren't as simple as other battery chemistries. This battery chemistry increases the level of difficulty and processing power for the control module because it now maintains ideal battery charging on top of MPPT.

\subsection{The Solution}

The highest level block diagram of the control system is shown below in Figure 1. The figure illustrates that the solar panel delivers power to a battery with a buck-boost converter having its duty cycle controlled by the control algorithm. The control algorithm analyzes the voltage and current from the solar panel and adjusts the duty cycle of the power converter to MPPT. The control algorithm also analyzes the battery's voltage and current, which is higher in priority, and adjusts the duty cycle to ensure the battery isn't breaching its maximum voltage or current ratings.

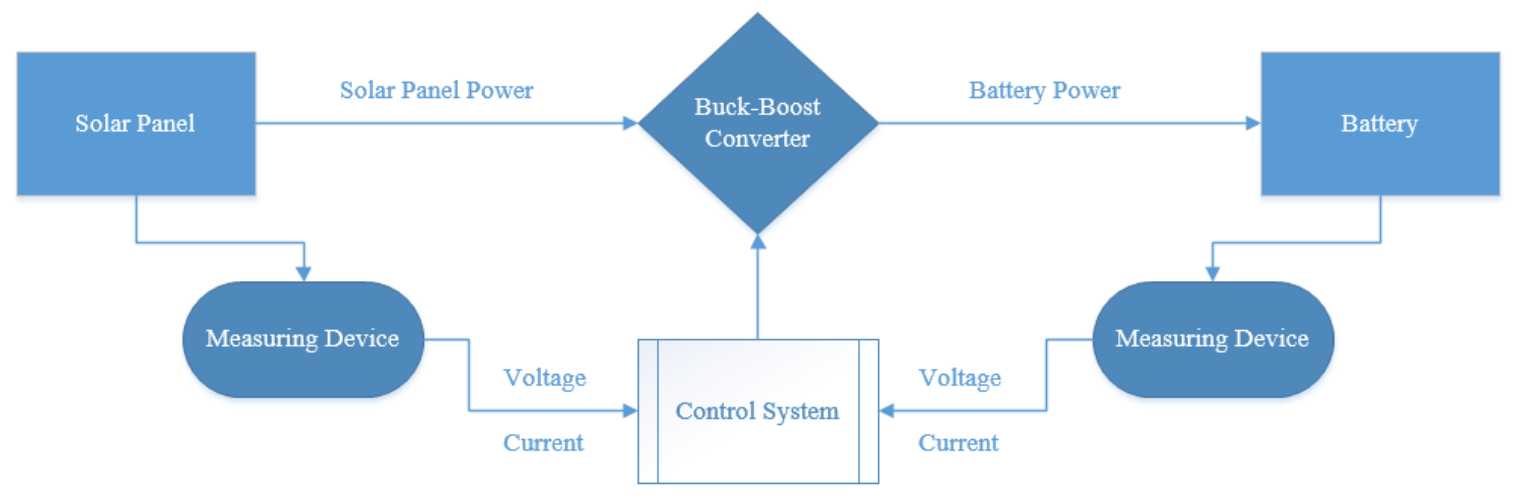

Figure 1. Highest Level Block Diagram of Control System

Creating models for the simulation and verification of the control system is extremely critical. The better and more precise the models are integrated, the stronger the algorithm. Simulink has become very powerful in the modeling of systems, and, therefore, it is used to 
develop the control algorithm. The models of physical components are for the solar panel, battery, power electronics, and control algorithm. The power electronics is modeled in Simulink; this greatly reduces the amount of inaccuracies in creating the complicated circuit (with switches) as a mathematical model in MATLAB program code. This approach to developing the algorithm can improve the current methods too; it's time efficient for test cases, the stability can be analyzed prior to implementation, and all components are adjustable.

\subsection{Chapter Summaries}

The remaining four chapters of the thesis contain: Literature Review, Background, Simulation and Results, and Conclusion and Future Work. Chapter 2 discusses the literature on control systems/algorithms for related charge controllers, MPPT for solar panels, and charging methods for lithium ion batteries. Chapter 3 contains the background information required to understand the physical dynamics of the solar panel, lithium ion battery, and power converter. In Chapter 4, the control system is broken down into block diagrams, the equations of the algorithm are expressed in variable parameters, the parameters are adjusted for different test cases, the parameters are determined via case to case, and the algorithm is compared to existing methods. Finally in Chapter 5, the control system is concluded and the possible future work for the modeling is presented. 


\section{Chapter 2. Literature Review}

In this section, research is reviewed and analyzed and the information about MPPT, charging Li+ batteries, and charge controllers doing both of these are discussed. This section is sought as the prime source of background information but with the credibility to the research.

\subsection{Solar Panel to Battery Chargers}

Solar panel to battery charging systems are typically highly confidential and the techniques between how they work is usually never released to public, however, there has been significant research done on a couple of different joint topologies in which to charge batteries with solar panels. The general idea to create a solar panel charger for a battery is joining the ideas between MPPT and effective charging onto the battery. There are several different chargers on the market with different advantages for other applications whom are a combination of the methods discussed in section 2.2 and 2.3. Many of the solar panel to lithium ion battery chargers are still not disclosable; however, there is research from lead-acid batteries.

\subsubsection{P\&O MPPT and CC/CV Charging for Lead-acid Battery}

In this paper ([11]), the P\&O algorithm MPP tracks a solar panel system. This algorithm is overridden via a charging algorithm using $\mathrm{CC} / \mathrm{CV}$. It uses $\mathrm{CC} / \mathrm{CV}$ because the panel's maximum power capability may be larger than the battery can accept. However the similarity to most chargers, this system uses a lead-acid battery as opposed to a Li+ battery. 


\subsubsection{Hill-climbing MPPT and CP/CV Charging for Li+ Battery}

This paper ([15]) researches using a hill-climbing algorithm on MPPT and charges the $\mathrm{Li}+$ battery with a $\mathrm{CP} / \mathrm{CV}$ technique. Their charging technique is the standard for such controllers; however, their tracking technique is different in the sense that they are using a hillclimbing algorithm versus the widely accepted $\mathrm{P} \& \mathrm{O}$ algorithm.

\subsubsection{Incremental Conductance MPPT and Three Charge Methods for Lead-acid Battery}

In this paper ([3]), they use incremental conductance MPPT with three different charging methods for lead-acid batteries. Once again, this system is utilizing lead-acid batteries, so their

charging techniques are different than those for $\mathrm{Li}+$ batteries. But, they only use incremental conductance to find the MPP, as opposed to using a hybrid type system to achieve greater results.

\subsubsection{P\&O MPPT and Charging for Lead-acid Battery}

This paper ([13]) is another investigation of the $\mathrm{P} \& \mathrm{O}$ algorithm with a lead-acid battery. They don't discuss their charging method, but it's assumed they are focusing on MPPT. Their abilities to MPPT are limited with the constant step sizes in the P\&O algorithm.

\subsection{Solar Panel Maximum Power Point Tracking Algorithms}

MPPT of a solar panel is an essential method to maximize the energy acquired from solar radiation. The MPP of a solar panel, shown in Figure 2, is where the solar energy being harnessed is at highest electrical efficiency. There is a significant amount of methods being utilized to MPP track solar panels in the industry and there are a couple extensively researched control methods: fixed voltage, fractional open circuit voltage, hill-climbing, P\&O, incremental 
conductance, voltage sweep/current sweep, fuzzy logic, and neural networks. All of these methods can be split into three different categories depending on their complexity and approaches: indirect based on solar panel characteristics, direct based on physical criteria, and direct based on abstract criteria.

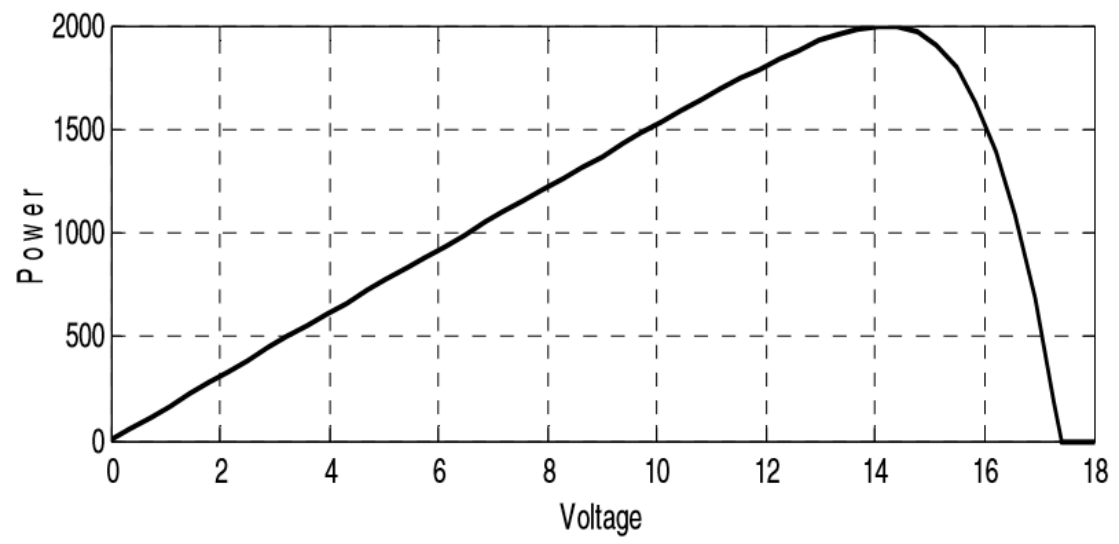

Figure 2. Representation of MPP for a Solar Panel [5]

\subsubsection{Indirect MPPT}

The indirect MPPT methods don't require measuring devices and are pre-optimized to the solar panel's characteristics and predicted environmental conditions. The two popular indirect methods for MPPT are the fixed voltage and fractional open circuit voltage schemes. These two control processes are known for their simplicity, but this isn't necessarily an overall advantage.

\subsubsection{Fixed Voltage}

The fixed voltage control algorithm is designed to maintain the solar panel at the same optimized voltage throughout any condition. The reason why this has been used is because the solar panel's MPP doesn't change too drastically based on voltage under many weather conditions and temperatures. The voltage is selected as the optimal voltage for the solar panel in 
its environment. The fixed voltage control algorithm follows the simplest process of all other MPPT algorithms; however, it has the largest drawbacks on actually tracking the MPP. It essentially always maintains the solar panel at a fixed voltage: though this voltage usually is not where the MPP is since solar panels always change their P-V curves during varying conditions. On the first day this algorithm may work optimally since the fixed voltage was set to that specific condition, but when clouds appear, the solar panel ages, or temperature changes, the MPP has changed and the algorithm may be ineffective or even useless. The advantages of using this method are that it's simple to implement, requires zero measurement devices, converges extremely fast to the voltage of ideal MPP, and is the most stable under any condition. The disadvantages are that it's not actually tracking MPP, can be completely useless, and needs consistent re-optimization or adjustments for new voltage values ([14]).

\subsubsection{Fractional Open Circuit Voltage}

The fractional open circuit voltage control algorithm determines the voltage to maintain on the solar panel based on an optimized scalar value of the relative open circuit voltage for any condition. This means that the algorithm measures the open circuit voltage and multiplies it with a scalar value to acquire the voltage to maintain the solar panel at. This method works well because a lot of research has shown that there is such a fractional scalar value that offers a close to MPP voltage. Essentially, when the P-V curve changes due to varying conditions in temperatures and irradiance, the open circuit voltage changes and the MPP voltage is nearly always the same fractional amount of it, as seen in Figure 3. The fractional open circuit voltage control algorithm incorporates a little more complexity then the fixed voltage method, but offers a much greater improvement in choosing a closer voltage to MPP. Some advantages this method 
offers are its quick convergence rates to a predicted optimal voltage, lack of measuring devices, and simplicity in implementation. The disadvantages are that it constantly needs to recheck the open circuit voltage, doesn't track the MPP, and needs consistent re-optimization or adjustments to its scalar value $([1,4,6,8,20])$.

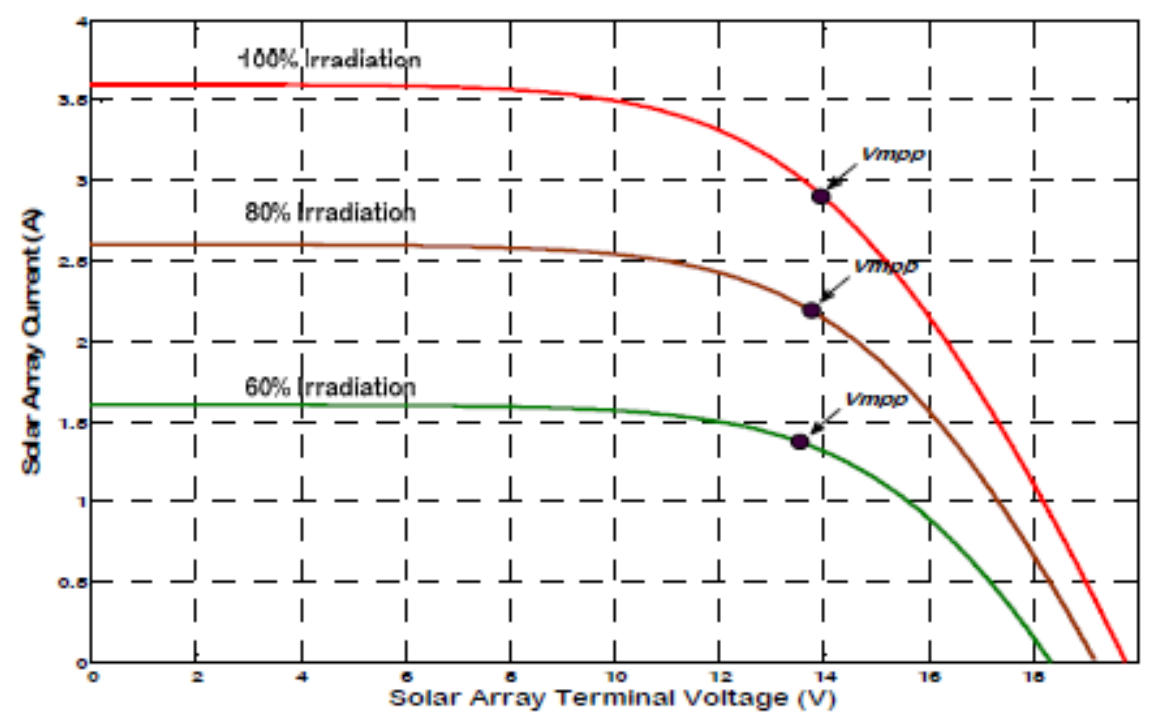

Figure 3. PV system for use of Fractional Open Circuit Voltage [1]

\subsubsection{Direct MPPT with Physical Criteria}

The physical-direct MPPT methods require measuring the current and voltage to decide what the next control phases are, and depending on the method, the devices may need accuracy and precision. These methods are physically direct in the sense that they are designed specifically for the P-V curve and its relationship to the tracking abilities. The most common methods to MPPT directly with physical criteria are hill-climbing, P\&O, incremental conductance, and voltage/current sweep methods. These methods are known for being robust and capable of MPPT in mostly every condition; however, depending on their operation frequency they are susceptible to dramatic weather changes. 


\subsubsection{Hill-climbing}

The hill-climbing control algorithm is the simplest of all physical-direct methods because it follows two rules: if power increases then increase duty cycle or if power decreases then decrease duty cycle (or visa-versa depending on the power converter), shown in Figure 4. Thus, there are two duty cycle step sizes that can be chosen. The P-V curve has two hills that can be climbed: from the short circuit current side or from the open circuit voltage side. The side chosen can affect the power converter differently, so it's respectable to correlate the direction and converter specifically. It's known as the hill-climbing method because it attempts to do its best to climb up the P-V curve towards MPP, but it doesn't have a steady-state error of zero and jumps around the sides of the MPP. Without the step size having variability, the MPP is most likely never achieved, but rather fairly close to it. Issues in the algorithm occur when weather changes too frequently; it can be misled into the incorrect direction because the P-V curve may change where the MPP is widely different than before. This issue can easily be fixed with running the algorithm at higher frequency. The advantages of this method are its simple implementation, general use on all solar panels, and ability to track MPP at varying rates. The disadvantages about this method are its limitation to a single step up or step down size, oscillation around MPP, and dependency on measuring devices accuracy and frequency ([2, 8, 10, 20]). 


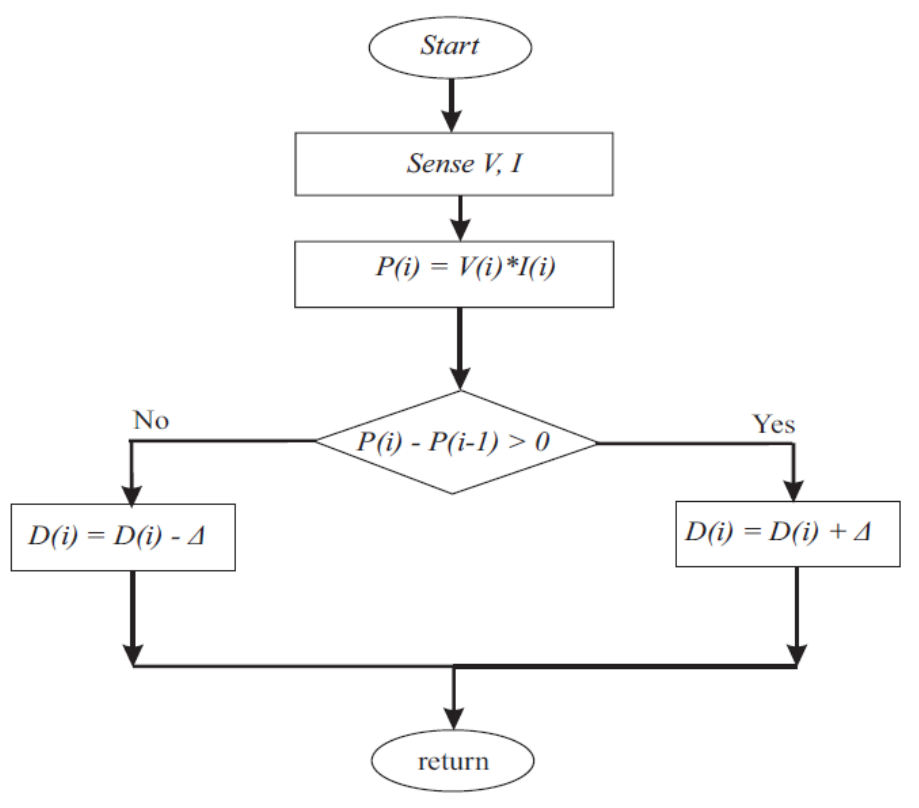

Figure 4. Hill-climbing Flowchart [10]

\subsubsection{Perturb and Observe}

The $\mathrm{P} \& \mathrm{O}$ control algorithm is very similar to the hill-climbing algorithm, they share nearly all the same advantages and disadvantages, but it also accounts for whether the voltage increased or decreased when the power changed. Knowing the direction the voltage changed with the direction the power changed gives more insight on the way to adjust the duty cycle. Instead of two cases to follow, there are four cases to follow, seen in Figure 5: if power increased and voltage increased then decrease duty cycle, if power increased and voltage decreased then increase duty cycle, if power decreased and voltage increased then increase duty cycle, or if power decreased and voltage decreased then decrease duty cycle (or visa-versa for all depending on the power converter). With the additional information being analyzed, there are four different duty cycle sizes to increase and decrease. Thus, depending on the side of the hill, it may be more advantageous to use smaller or larger duty cycle increments. This ultimately allows lesser ripple 
at MPP as opposed to the hill-climbing method. This is the most widely adopted method in the industry because of it intuitive sense and robust convergence to MPP. However, creating a varying size on the duty cycle increments further improves the method. This method is also susceptible to fast changing weather conditions, though it can be solved with higher frequency updates. The advantages of this method are the low complexity, consistent converging rates to MPP, and ability to step in the correct direction a specific amount dependent on the side it's climbing. The disadvantages are its inability to have zero steady-state error at MPP, four fixed step sizes, and dependency on measuring device's characteristics $([5,7,8,10,20,21])$.

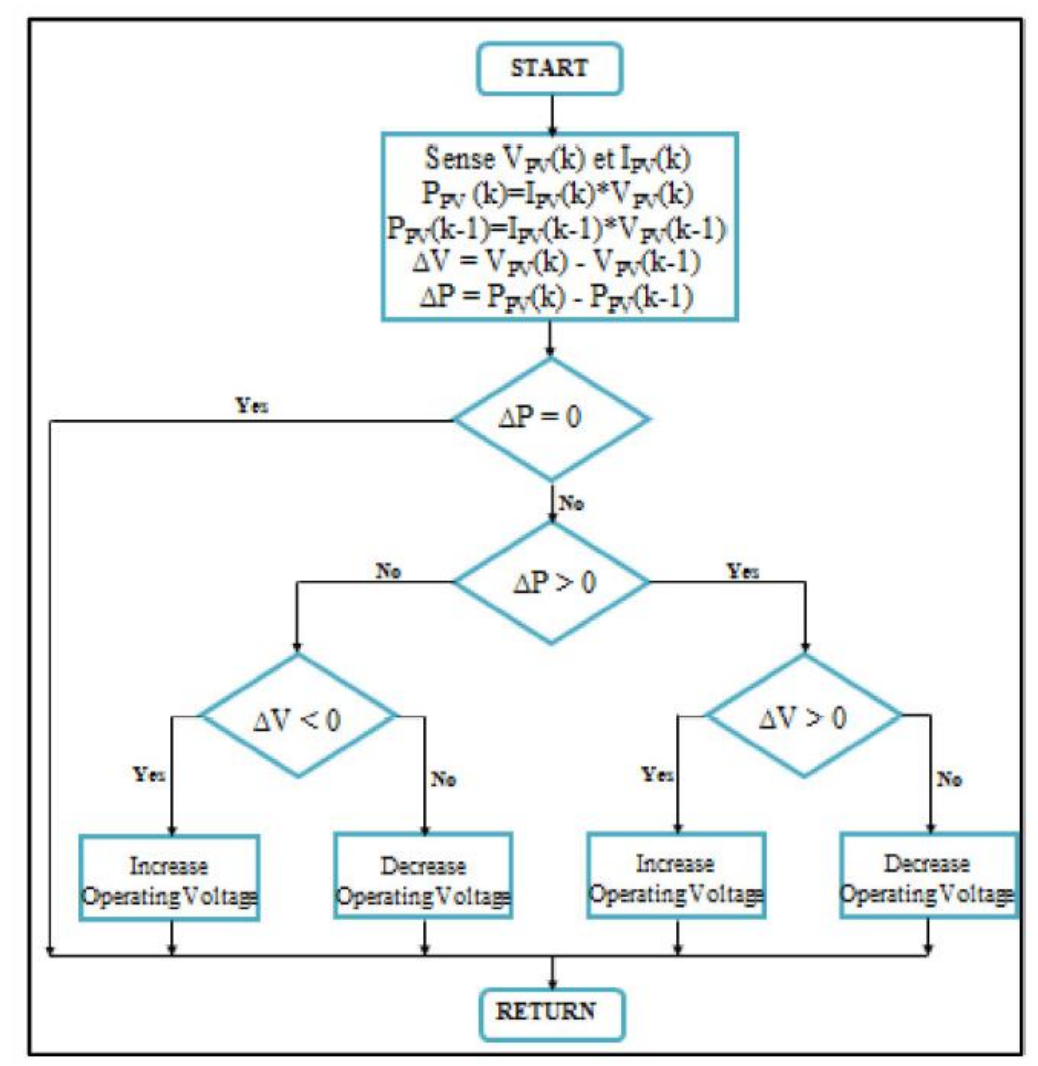

Figure 5. P\&O Flowchart [21] 


\subsubsection{Incremental Conductance}

The incremental conductance control algorithm tracks MPP through utilizing the change in power relative to the change in voltage (i.e. $\mathrm{dP} / \mathrm{dV}$ ). The $\mathrm{MPP}$ is where $\mathrm{dP} / \mathrm{dV}$ is equal to zero, where the two climbing hills meet. Because this algorithm is able to acquire zero steady-state error after it converges towards MPP. The rules this algorithm follows is to increase duty cycle when $\mathrm{dP} / \mathrm{dV}$ is less than 0 or decrease duty cycle when $\mathrm{dP} / \mathrm{dV}$ greater than 0 (or visa-versa depending on the power converter), portrayed in Figure 6. Depending on the size of $\mathrm{dP} / \mathrm{dV}$ the step is larger or smaller, sometimes directly scaled to the $\mathrm{dP} / \mathrm{dV}$ itself (i.e. the adaptive incremental conductance method). Initially in the $\mathrm{dP} / \mathrm{dV}$ curve the magnitude is positive then crosses pass zero and finishes with a high negative magnitude. Thus, as the MPP voltage is approached the duty cycles tend to decrease to smoothly reach the MPP. When the MPP is acquired, the duty cycle is no longer adjusted and the voltage remains the same, giving zero steady-state error. Though, if weather conditions change, the tracking to the new MPP may be quite slow because the duty cycle is incrementing very small. This algorithm is sometimes chosen over P\&O because it has lesser oscillations around MPP. The advantages of this method are its physical meaning to tracking MPP, small if any oscillations around MPP, and variable duty step size. The disadvantages of this method are the inability to occasionally track a new MPP, complexity of implementation, and dependency on measuring devices accuracy and frequency $([8,10,12,20,21])$. 


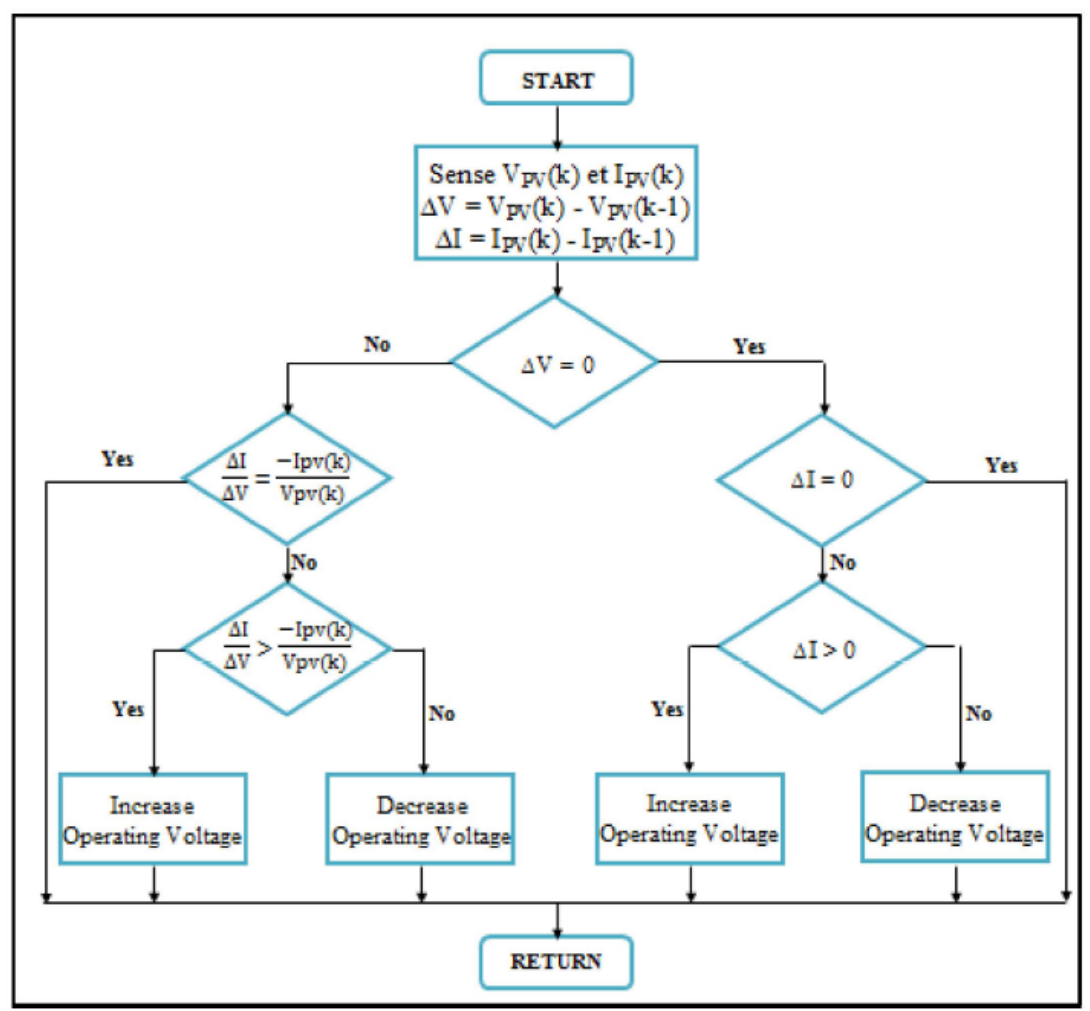

Figure 6. Incremental Conductance Flowchart [21]

\subsubsection{Voltage/Current Sweep}

The voltage sweep control algorithm slides across all voltage values and measures the power relative to each voltage, then determines where the MPP is and maintains it (this can be directly correlated with the current sweep method). Every time the MPP changes the algorithm won't track it until it slides across voltage values again to acquire the new voltage value. There initially is zero steady-state error, but the re-evaluation phase for the MPP voltage value can create a large amount of inefficiency. This is the least likely used method of the four physicaldirect methods because it doesn't exactly track the MPP but rather finds it every now and then. However, it has the quickest convergence times of all the physical-direct tracking methods right after it scans across the voltage values. This algorithm is sometimes limited to a specific window 
size of voltage values where MPP always is; this increases its speed and allows for more sliding iterations without reducing efficiency. This method performs well in areas where climate change is highly unlikely throughout the day and can scan precisely when drastic weather changes occur. This algorithm can be sought as very powerful since it works with all solar panels and doesn't require recalibration, unless a chosen voltage window is selected. The advantages of the method are its ability to determine MPP in a single sweep, initial zero steady-state error, and ease of power usage. The disadvantages of this method are its inability to MPPT, constant resetting to acquire a new MPP, and dependency on measuring devices accuracy and frequency ([8, 20]).

\subsubsection{Direct MPPT with Abstract Criteria}

The abstract-direct MPPT methods also require measuring the current and voltage, similarly to physical-direct MPPT, to determine the next action. These methods are abstractly direct because they are based on abstract concepts that can acquire MPPT pristinely without some faults of the physical-direct methods; however, they are optimized for certain situations similar to the indirect MPPT methods.

\subsubsection{Fuzzy Logic}

The fuzzy logic control algorithm is designed to track the MPP utilizing its fuzzification, rules, inference system, and defuzzification. Basically, there is always uncertainty in the algorithm and it uses this uncertainty, known as error, to determine the next step towards the MPP. This algorithm works based on a set of rules and membership functions, shown in Figure

7, for an inference system. During certain situations, different rules are triggered and this ultimately allows the algorithm to MPP track. The first thing is to determine the rules for a 
specific solar panel, then optimize the weights of the rules. If the rules are created thoroughly and the error signal is very clear, then this method is capable of quickly converging to the MPP and maintaining it without large oscillations. However, once the fuzzy logic is designed for a specific solar panel it's most likely not adequate for another solar panel in the optimal way because many of the rules and factors need re-adjustments. This method is very strong initially, but as the solar panel degenerates and climates change, the algorithm is no longer optimized for that solar panel. The advantages of this method are its ability to quickly track MPP, maintain MPP without hovering, and dynamically approach a new MPP. The disadvantages of this method are its optimization to a specific setting, dependency on its error calculation, and significantly high complexity $([2,5,8,10,17,20])$.

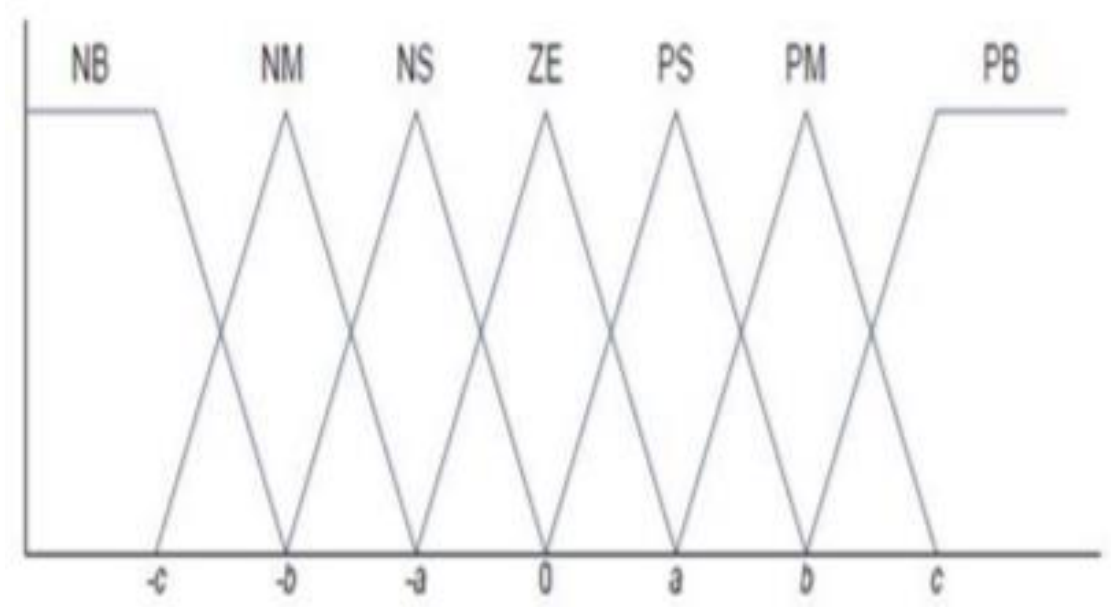

Figure 7. Example of Typical Membership Functions [5]

\subsubsection{Neural Network}

The neural network control algorithm uses layers of interlinked scalar values, Figure 8, that have been trained to MPP in order to track the MPP based on voltage and current inputs. This method utilizes the power of mathematics and correlation between abstract variables to determine where the MPP is. In order to train neural networks, significant data is first collected 
upon the solar panel to maximize its use. If the data is sufficient, then this method could perhaps be the most powerful of all the methods, however, acquiring such sufficient data is very tedious and highly unlikely. The data that is necessarily to acquire is the relationship between the change in duty cycle based on voltage and current inputs. Obtaining large enough data sets may even require simulation of the physical model since the time is limited. This neural network is optimized specifically for the data it has been trained on, so other conditions that may arise, it won't be able to efficiently re-accustom to. Therefore, it is particularly limited to the data set to which is trained with. Also, it is highly dependent on the amount of layers and number of neurons chosen for each layer. Thus, there is uncertainty if the correct number of each is chosen to reduce over modeling or under modeling the situation. This method is quite new and there isn't much research on optimization factors as researchers are still studying and learning about how the neural networks communicate. The advantages of this method are its ability to track MPP in all trained conditions, low oscillations around MPP, and completeness of grasping all aspects of a solar panel. The disadvantages are its need for retraining, absolute abstract processes with uncertainty, and the extremely high complexity $([8,10,16,20])$.

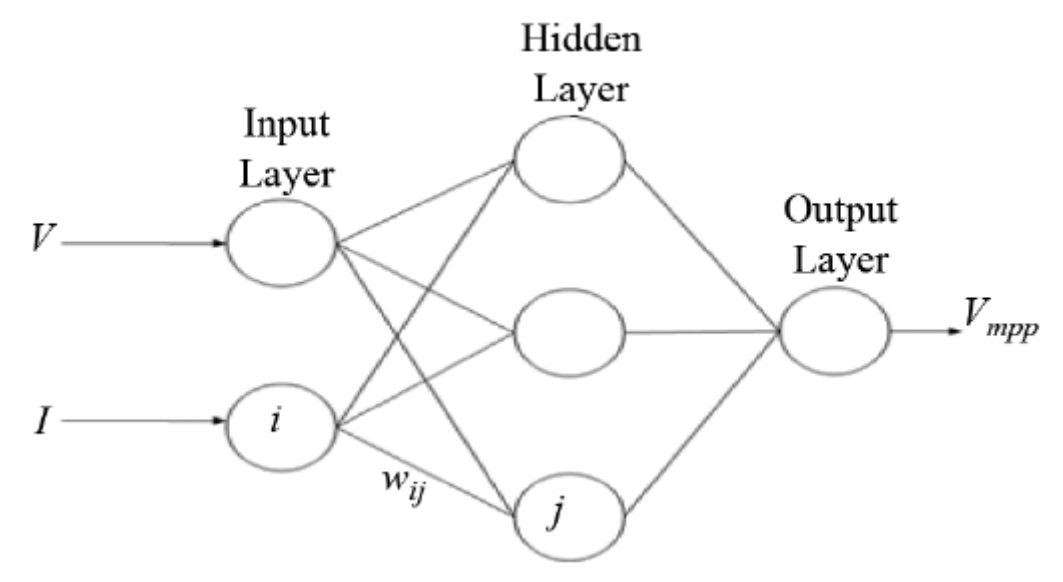

Figure 8. Example of a Neural Network [20] 


\subsection{Lithium Ion Battery Charging Algorithms}

There are a couple different ways to charge lithium ion batteries; however, the most robust and widely accepted way of charging is the constant current to constant voltage $(\mathrm{CC} / \mathrm{CV})$ method. The few others consist of pulse current charging, multi-stage current charging, and constant voltage charging. These methods were researched in comparison to $\mathrm{CC} / \mathrm{CV}$, and offer different advantages and disadvantages depending on the charging system.

\subsubsection{Pulse Current}

Pulse current charging is performed by sending pulses of current to the battery with a specific current level, and as the battery gets closer to full charge, the pulses become less in time length, shown in Figure 9. This is a very simple yet versatile way to charge the battery without over charging. When charging systems are limited to a single rate of current output, they are still capable of charging the battery; however, they require the ability to adjust the pulse length. Though this method can be used, it can easily cause the battery to overshoot its maximum voltage when it is near end state of charge; thus, harming the battery and potentially destroying the electrochemical reaction through over polarization. The advantages of this charging technique is its simplicity and that it can charge a battery full on a single current. The disadvantages are the pulse widths which require adjustments according to the relative battery voltage and the lack of maintaining a steady voltage on the battery during end charge ([18, 22]). 


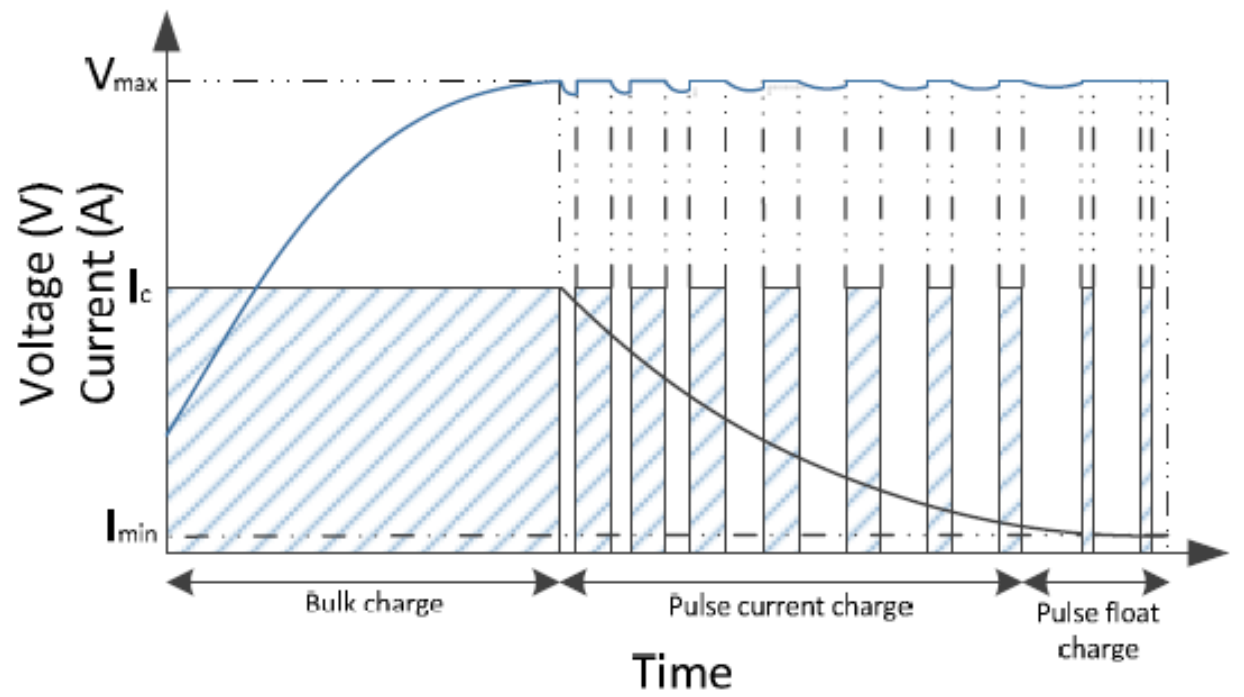

Figure 9. Pulse Current Charge [18]

\subsubsection{Multi-Stage Current}

Multi-stage current charging has different levels at which the charging system may charge at, as seen in Figure 10. When the battery is charged at a single level for an extended period of time and reaches max voltage, the next lower stage of current is applied until the battery achieves max voltage again; and continues to do this until the lowest current stage is achieved. This method of charging the battery is technically safer than the pulse charging in the sense that it won't push the voltage over the voltage limit and instead steps to a new current value. The advantages of this method are that it is simple to implement, charges batteries safely, and can operate on discrete charging systems. The disadvantages of this charging technique are that it doesn't charge the battery quickly to maximum voltage and lacks the ability to maintain the battery at maximum voltage during the end charging phase ([18, 22]). 


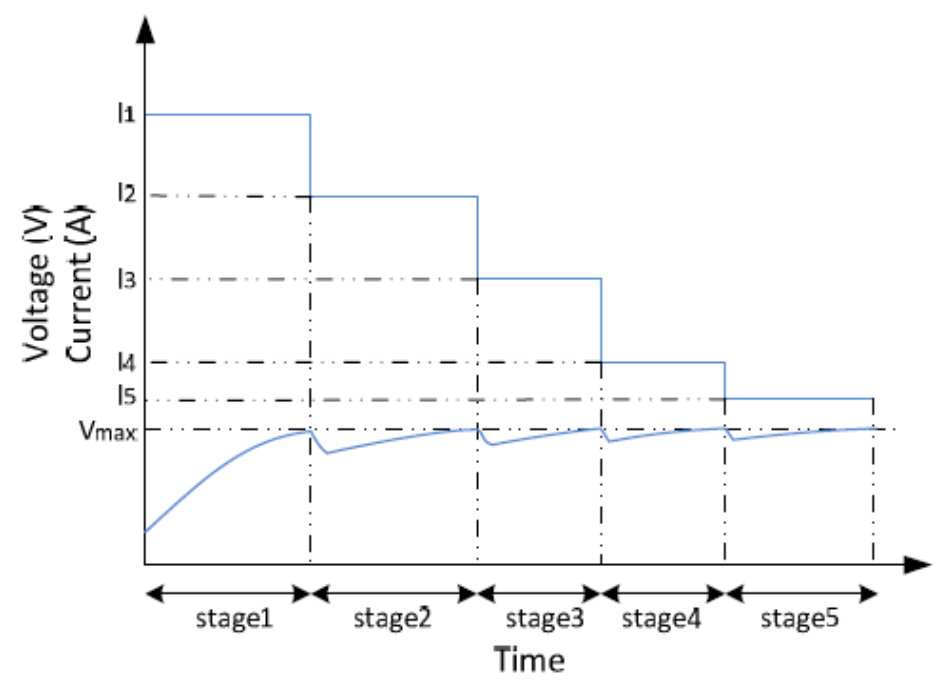

Figure 10. Multi-Stage Current Profile [18]

\subsubsection{Constant Voltage}

Constant voltage charging puts a voltage across the battery that is maintained depending on the speed the battery requires to charge. Sometime this voltage is higher than the voltage of the batteries optimal value to boost charge the battery; however, this can force a lot of current and potentially harm the properties of the battery. Relatively, it is good to charge the battery up to its maximum voltage when the current being drawn doesn't cause thermal reactions disrupting electrochemical properties. Using multiple stages of constant voltages to maintain reduces the chances of destroying the battery, however this often results in longer charge durations; hence why there's a tendency to put larger voltage values across the battery. When the battery begins to get near end of charge, the optimal battery voltage is put across the battery to acquire it and not over charge. The advantages of this system are the simplicity of implementation, the quickness of charge, and variability settings. The disadvantages of this charging method are its inability to charge the battery under current conditions, the potential harm it may cause during charge, and the varying power output onto the battery $([18,22])$. 


\subsection{4. $\mathrm{CC} / \mathrm{CV}$}

$\mathrm{CC} / \mathrm{CV}$ charges the battery at constant current and then at constant voltage, displayed in Figure 11. This method is the most robust and offers the safest result when charging a battery because it doesn't push too much current through the battery and it doesn't put too much voltage across the terminals. This method can sometimes be slower than others because the end of charge phase with constant voltage can be extremely lengthy if that voltage is the voltage the battery wants to charge to; this is fixed by slightly increasing the constant voltage value so more current is inputted, but this again can cause harm to the battery. This charging technique offers charging under controlled systems and requires a device that can charge at a constant current and constant voltage, unlike the other three methods. The advantages of this method are its ability to charge the battery at a steady rate quickly to $80 \%$, safe power forced upon a battery, and robustness. The disadvantages are its limitations to quickly charge the battery at end of charge, multiple output and input devices, and increasing power on the battery during constant current phase. A potential alternative to this method is $\mathrm{CP} / \mathrm{CV}$ when the battery can accept maximum power output from the charging system $([9,18,19,22])$.

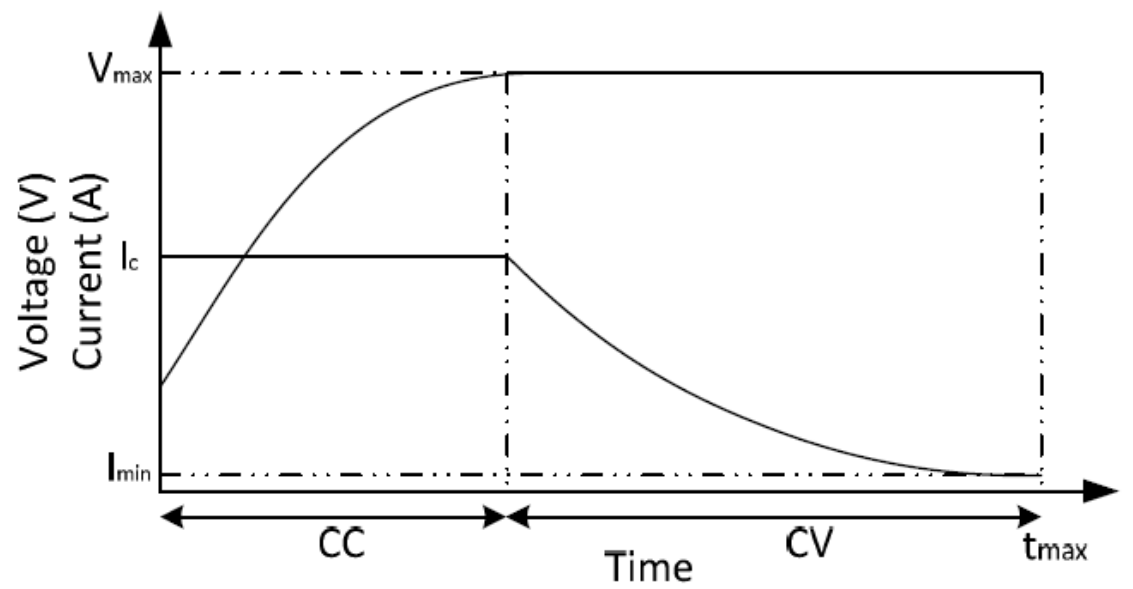

Figure 11. CC/CV Profile [18] 


\section{Chapter 3. Background}

This chapter contains the additional supplemental information that complements the information from Chapter 2. This information is necessary for understanding the models in Appendix A that are for the simulation of the physical systems: solar panel, lithium ion battery, and non-inverting buck-boost converter.

\subsection{Solar Panels}

Solar panels have an open circuit voltage, a short circuit current and a MPP. As the irradiance goes down, the amount of current supplied reduces as well. Depending on the solar cells, the max power occurs at different voltages for varying irradiance. Figure 12 shows a solar panel's current and voltage relationship against irradiance levels. Because the current is changing proportionally in this example, the MPP always remains located at the same voltage. This may seem like a very simple solution, always run the solar panel at nominal voltage; this is not the case however with many solar panels, as the power to voltage curve may shift inwards or outwards depending on the irradiance, previously shown in Figure 3. Therefore, a more robust control system is necessary if the solar model has a shifting power to voltage curve.

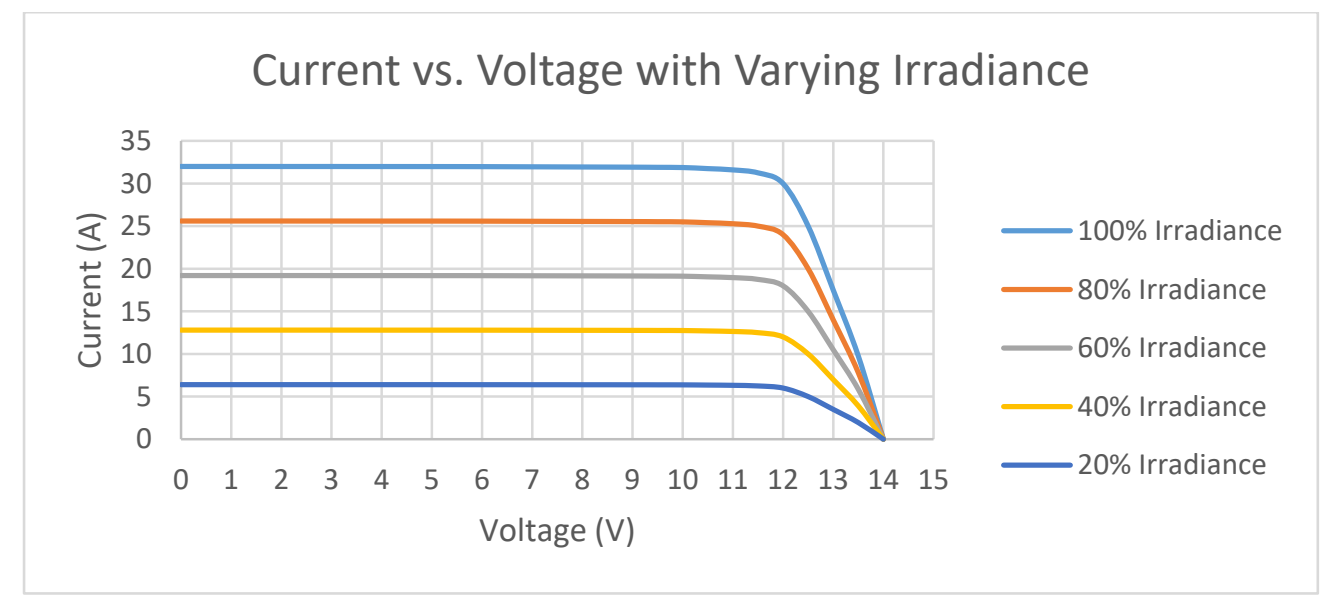

Figure 12. Current vs. Voltage with Varying Irradiance 
Due to these characteristics of the solar panel, the slope of the power-voltage curve is much greater on the right hand side, open circuit voltage to MPP, than the left hand side, short circuit current to MPP. Therefore, larger voltage step sizes are essential to have equal convergence rates to MPP from either open circuit voltage or short circuit current. In practice, MPP is approached from the open circuit voltage so short circuit current isn't flowing through the power converter. In general, a solar panel can be realistically represented as a look up table on the order of one, two, or even three different variables: voltage, irradiance, and temperature.

\section{2. $\mathrm{Li}+$ Batteries}

The model shown in Figure 13 is a typical circuit representation of a lithium ion battery; it's referenced as the dual polarization (DP) model and is a widely accepted model for this battery chemistry. The battery model is known as the DP model because it represents both the electrochemical polarization and the concentration polarization; though, many more parallel RC circuits can be added in series to create a more accurate model, doing so increases complexity and computational power. This model is simple in a way because it can be expanded upon to explain many other features (e.g. hysteresis, self-discharge, state of health, etc.) of the Li+ battery chemistry. On the contrary, this model may be considered complex because it utilizes two exponential functions as supposed to a single; with two exponential functions to define the battery, it now turns into a non-linear equation, with only possible solutions calculated through iteration. Though this, series resistance can be solved easily just like the open circuit voltage can when the battery is at resting conditions. The maximum voltage of most $\mathrm{Li}+$ batteries is around $4.2 \mathrm{~V}$ so the internal chemistry is at ideal polarization for reliable energy storage. 


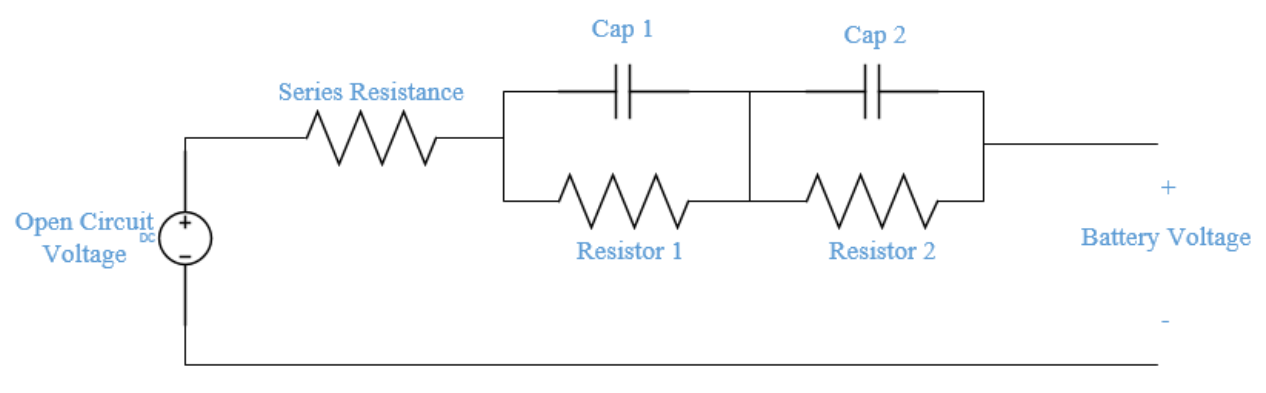

Figure 13. Equivalent Circuit of a Li+ Battery

In Figure 14, a battery's voltage is shown on the left. The battery is being discharged at a constant current, and then the discharge stops to reveal a depolarization curve. This depolarization curve can be fitted using 1,2,3, or up to an x number of parallel RC circuits; however, each additional RC circuit is another scaled exponential function. When the battery remains rested for extended periods of time (greater than two hours is reasonable for certain chemistries) the Open Circuit Voltage (OCV) can be measured. Finally, a large current pulse through the battery creates a large voltage drop; this instantaneous voltage drop is directly related to the series resistance (Rs) since the other RC circuits are shorted across their capacitor at this instance. All these parameters change when the battery is at a different state of charge; thus, extracting the parameters at multiple state of charge (SoC) significantly enhances the representation of the battery. OCV generally increases linearly as SoC increases from 20\%-80\% and $\mathrm{OCV}$ tends to exponentially increase to $4.2 \mathrm{~V}$ as SoC increases from $80 \%-100 \%$. The parameters of the circuit are then extracted via fitting the data to experimental results of the battery's voltage response to the input current. 


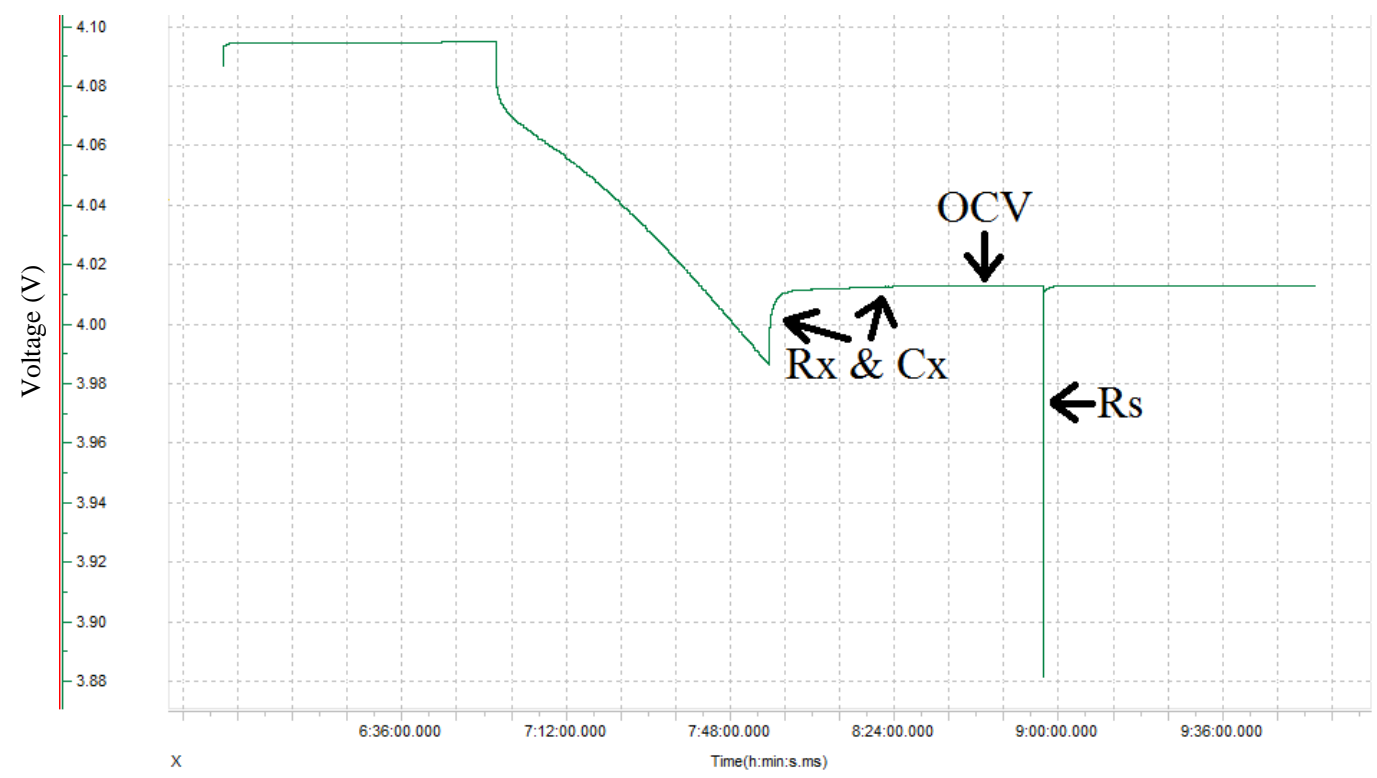

Figure 14. Parameters of Li+ Battery Equivalent Circuit

\subsection{Power Converter}

This electronic circuit in Figure 15 shows the non-inverting buck-boost converter topology. Relating back to Figure 1, the solar panel is the input on the left, the battery is the output on the right, and the converter is in the center. This is a classical high efficiency converter and is fairly straight-forward in control. Switch 1 is known as the buck switch because when pulse width modulating (PWM) is used to control switch 1, the voltage is bucked more as the duty cycle decreases. To operate in boost mode, switch 1 stays on and switch 2 turns on with a different PWM duty cycle; the higher the duty cycle, the higher the boost. Despite these fundamental aspects, the inductor ideally maintains the current the solar panel has at max power; therefore, the switches are sent PWM signals in a proper manner to achieve maximum energy transfer without harming the battery. 


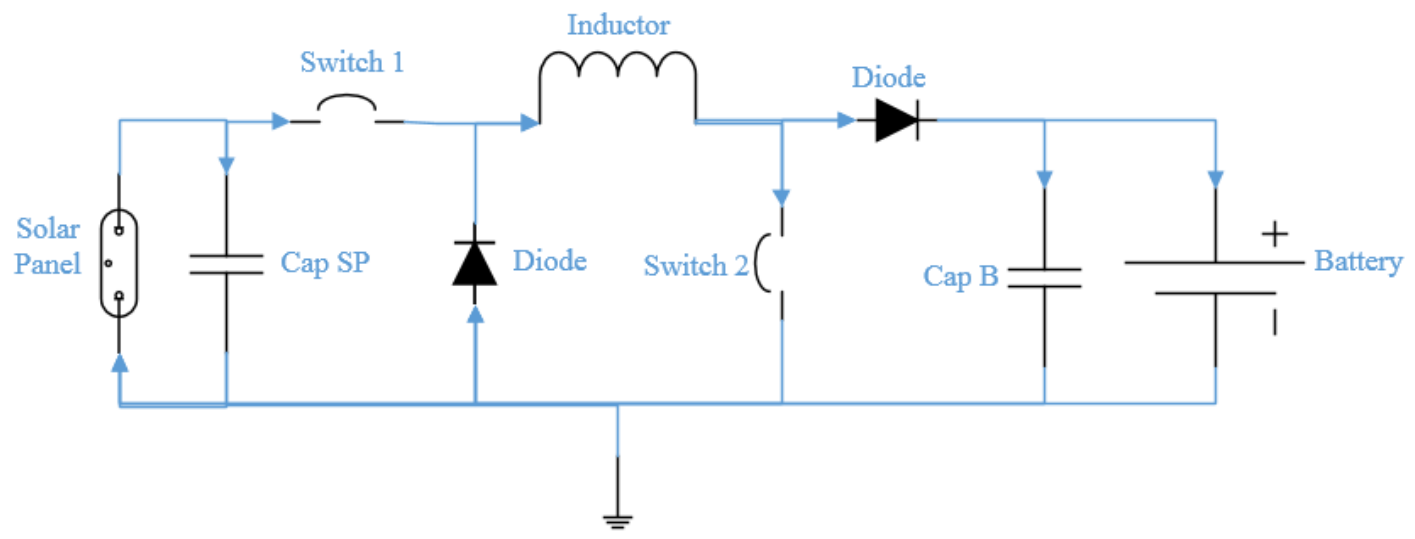

Figure 15. Model of a Non-Inverting Buck-Boost Converter 


\section{Chapter 4. Simulation and Results}

This chapter covers the operation of the control system, its adjustable equations and the chosen parameters for the equations, and a comparison to previous techniques. The models for the complete physical system are found in Appendix A and several parameter simulations are contained in Appendix B.

\subsection{The Proposed Approach}

In order to produce a new algorithm, the physical systems need correct modeling or else the simulation won't realistically reflect their properties and characteristics. The first goal was to create a high level block diagram of the systems and their communicating signals; this diagram is illustrated in Figure 1. A realisticly modelled simulation is comprised of a solar panel delivering power to a battery through a controllable non-inverting buck-boost converter. The control system analyzes both the solar panel's and battery's voltage and current in order to send proper commands to the converter. Unlike other methods, the proposed approach is combining both $\mathrm{P} \& \mathrm{O}$ with adaptive incremental conductance and using a $\mathrm{CP} / \mathrm{CV}$ charge technique. The hybrid method reduces the limitations of accuracy in MPPT as opposed to the P\&O method. Also, this technique is designed to improve the convergence rates and steady-state of MPPT relative to those overviewed in section 2.2.

Simulations are performed in MATLAB since it can simulate the entire system shown in Figure 1. The Simulink features are solely used for the MATLAB simulations. Simulink visually represents the operations and signal routing of program code and even allows for circuits (powerlib library) similar to LT Spice, but compatible with Simulink. Simulink is good for the power converter circuits because the complex models don't require the elements coded in 
software; plus, the approximations are too large when representing switches in classical code. Everything else (solar panel, $\mathrm{Li}+$ battery, and control algorithm) is capable of being represented in Simulink because they are well defined in look up tables and Simulink blocks. The solar panel model (section A1.1) and Li+ battery model (section A.1.2) use data from their realistic characteristics; thus, the control system design is based around the chosen realistic factors.

\subsubsection{The Control System}

As seen in Figure 1, Figure 16 is the internal block diagram of the control system. There are two different delta duty cycles to select and the least value delta duty cycle is chosen. One delta duty cycle signal, Delta Duty Cycle MPPT, depends on the solar panel's voltage and current, whereas the other delta duty cycle signal, Delta Duty Cycle Critical, depends on the battery's voltage and current.

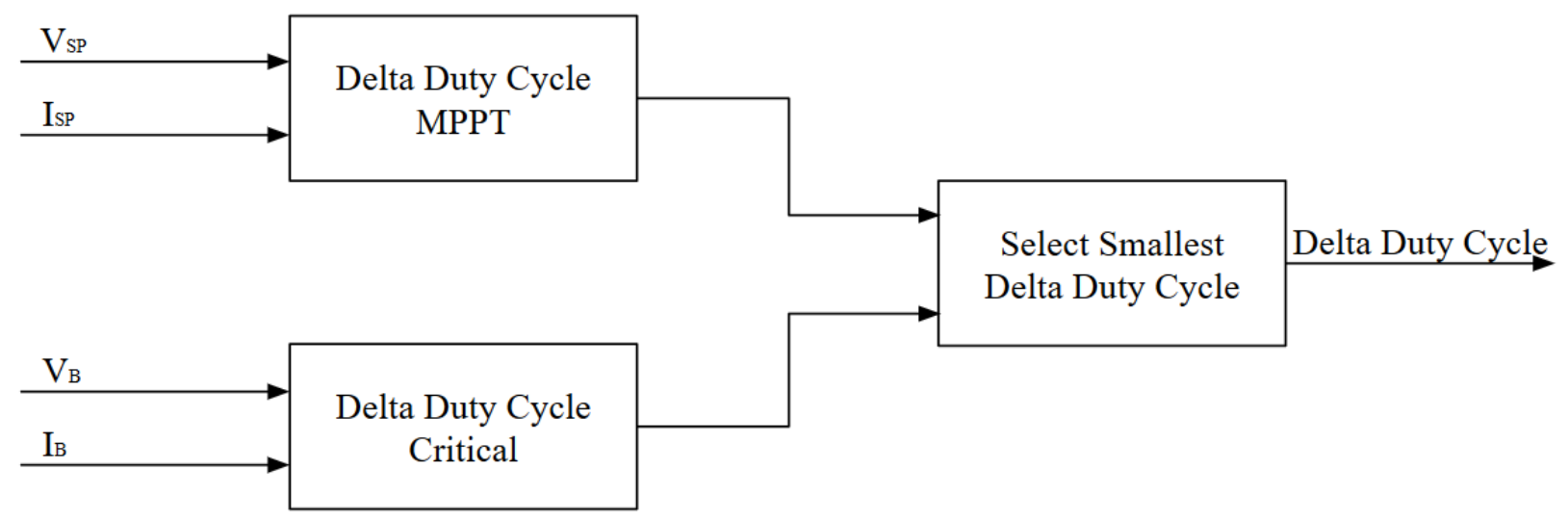

Figure 16. Block Diagram for Delta Duty Cycle

Equation 1 calculates Delta Duty Cycle MPPT, $\Delta D_{M P P T}$; which MPP tracks the solar panel with the hybrid method of adaptive incremental conductance and P\&O. Variables $a$ and $b$ are adjustable parameters; where $a$ is the scalar for the adaptive incremental conductance method 
component, $\frac{d P}{d V}$, and $b$ is the step size for $\mathrm{P} \& \mathrm{O}$. Because the power-voltage curve on the solar panel is different on each side of MPP, $a$ and $b$ may both be separated into $a+, a-, b+$, and $b$ - to improve the quality of convergence depending on which side the algorithm is climbing the solar panel's power hill. The $a+$ and $b+$ parameters are for increasing duty cycle, whereas, the $a$ - and $b$ - parameters are for decreasing the duty cycle.

$$
\Delta D_{M P P T}=a \frac{d P}{d V}+b
$$

Figure 17 expands the block Delta Duty Cycle MPPT from Figure 16. The derivative of power in terms of voltage $(\mathrm{dP} / \mathrm{dV})$ for the solar panel is used to determine if the duty cycle must increase or decrease (relative to the incremental conductance conditions); and results in calculating equation 1 for the Delta Duty Cycle MPPT signal.

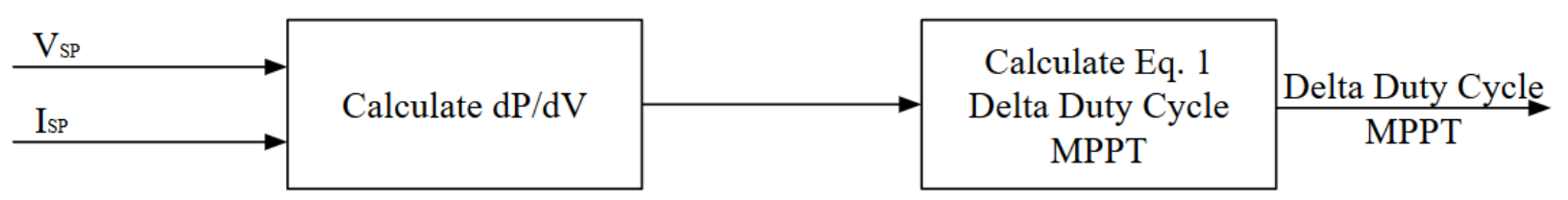

Figure 17. Block Diagram for Delta Duty Cycle MPPT

Equation 2 calculates Delta Duty Cycle Critical Voltage, $\Delta D_{V}$; which converges the battery's voltage to its maximum rated voltage. Variables $c$ and $d$ are adjustable parameters; where $c$ is the scalar for the error in voltage element, $\frac{\left(V_{\max }-V_{B}\right)}{V_{\max }}$, and $d$ is the scalar for the change in battery voltage (between time steps) element, $\Delta V_{B}$.

$$
\Delta D_{V}=c \frac{\left(V_{\max }-V_{B}\right)}{V_{\max }}+d\left(\Delta V_{B}\right)
$$


Equation 3 calculates Delta Duty Cycle Critical Current, $\Delta D_{I}$; which converges the battery's current to its maximum rated current. Variables $e$ and $f$ are adjustable parameters; where $e$ is the scalar for the error in current element, $\frac{\left(I_{\max }-I_{B}\right)}{I_{\max }}$, and $f$ is the scalar for the change in battery current (between time steps) element, $\Delta I_{B}$.

$$
\Delta D_{I}=e \frac{\left(I_{\max }-I_{B}\right)}{I_{\max }}+f\left(\Delta I_{B}\right)
$$

Figure 18 expands the block Delta Duty Cycle Critical from Figure 16. There are two separate equations: one for a critical voltage case, equation 2, and one for a critical current case, equation 3. The more critical case of the two, which is the smaller delta duty cycle, is chosen as the Delta Duty Cycle Critical signal; this signal maintains the safety and health of the battery.

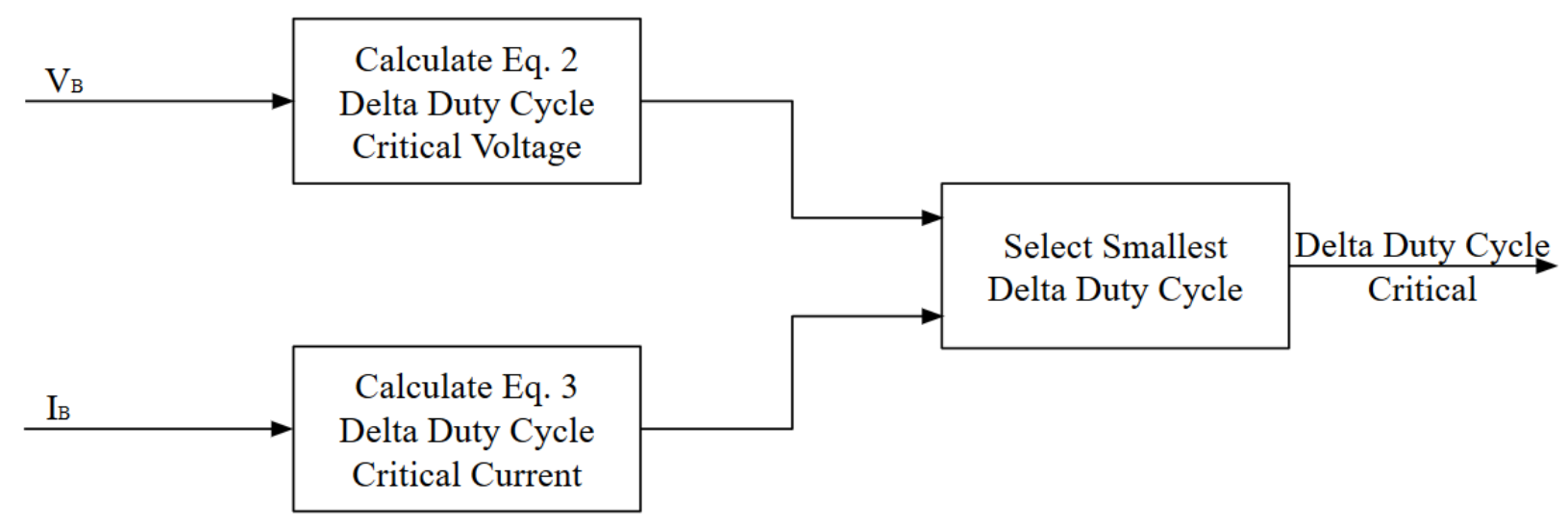

Figure 18. Block Diagram for Delta Duty Cycle Critical

\subsection{Test Cases}

There are three deterministic situations of how to increase or decrease the solar panel's power: maximizing solar power, maintaining max battery voltage, and maintaining max battery current. 


\subsubsection{MPPT Control Test Case}

The first case is to maximize solar power; Figure 19 shows an example of MPPT using only adaptive incremental conductance. The system is tracking the MPP and when the voltage and current of the battery aren't critical, the battery can charge at full efficiency via the solar panels max power. The maximum capable power is at $3 \mathrm{~W}$ (the top signal), and it's shown below that the system is tracking maximum possible power output as the duty cycle of the boost control (Duty Cycle 2) approaches the ideal duty cycle (shown as the bottom signal). In this example, the duty cycle struggles to change dynamically on the downhill because the power-voltage curve has a smaller slope on the current dominated side; thus, the convergence is slower using one parameter for $a$. The battery's initial SoC is $50 \%$. More test cases are in section B.1.

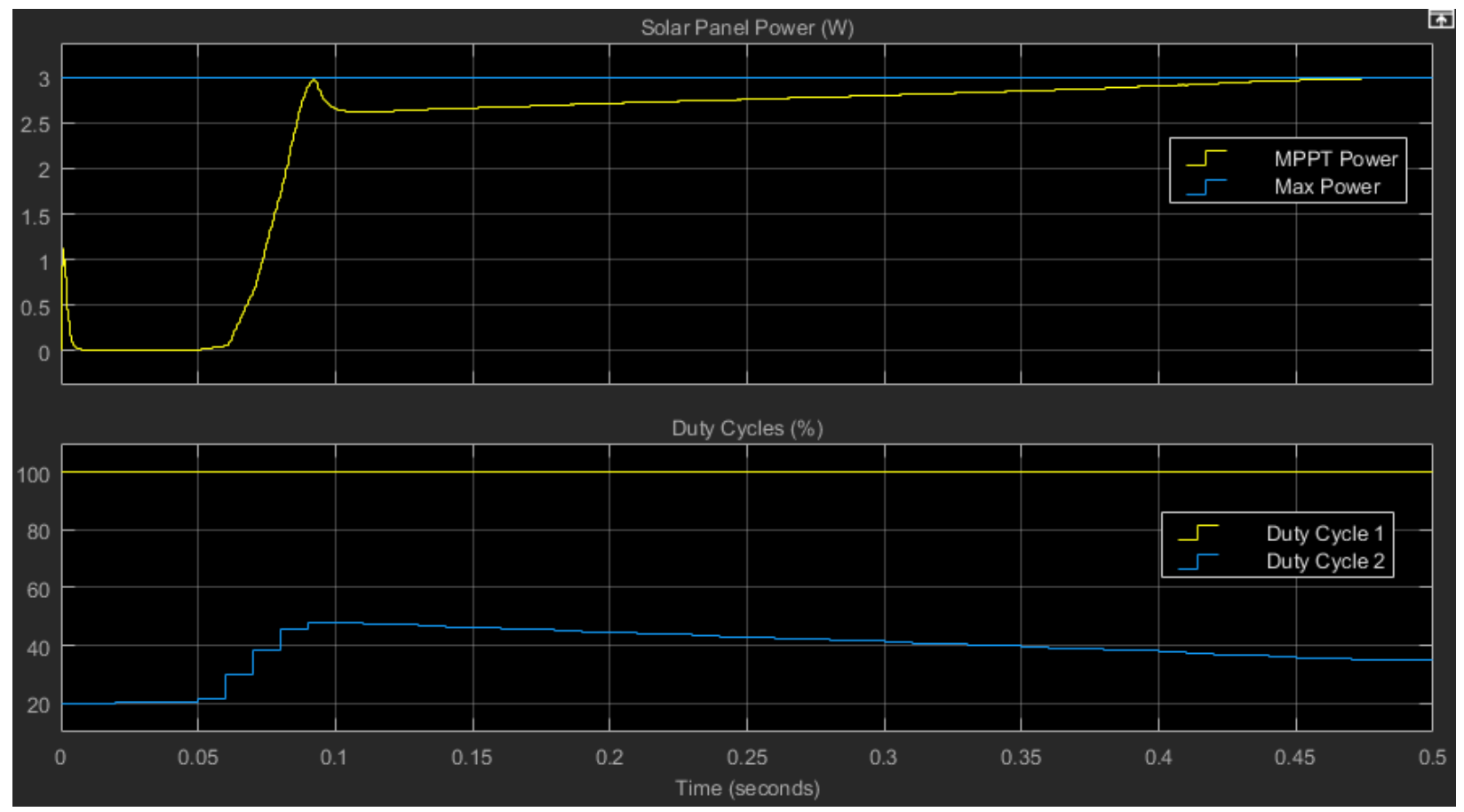

Figure 19. Example Case: $a=-0.005, b=0$ 


\subsubsection{Battery Voltage Control Test Case}

In this next case, Figure 20, the max rated voltage level for the battery is set at $4.2 \mathrm{~V}$. The top signal is the voltage of the battery and the constant line represents the limit. There is a clear oscillation around the limit and this is due to a lag between the controls and the measurement values; this also represents the fact that there is only proportional control. If the derivative element was included, the voltage level would have smaller oscillation about the maximum limits. The battery's initial SoC is $99 \%$. Additional test cases are in section B.2.

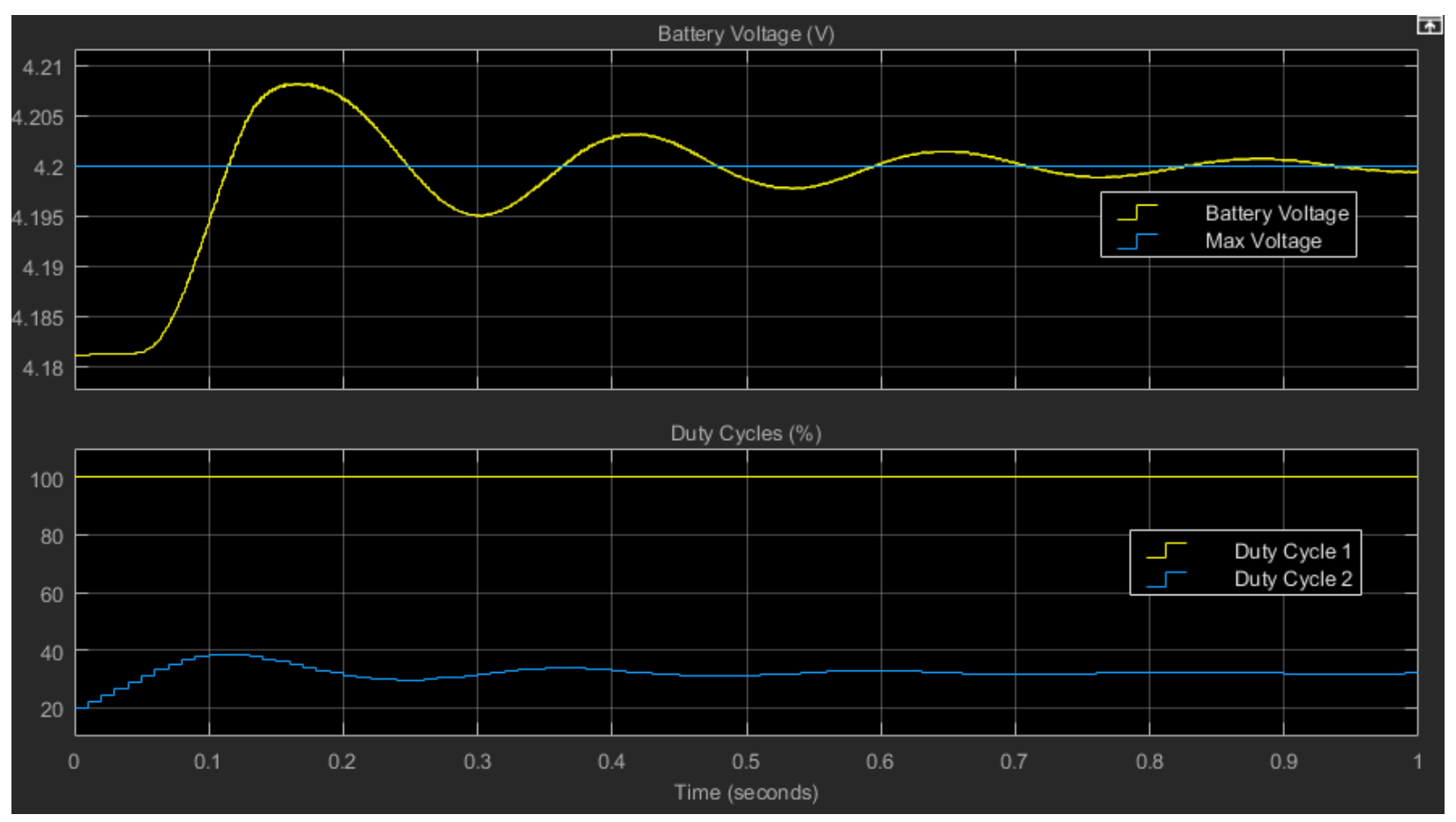

Figure 20. Example Case: $c=5, d=0$

\subsubsection{Battery Current Control Test Case}

The third case, Figure 21, is when the battery's maximum rated current is set to 0.5A. The derivative element is deactivated to show the oscillations due to the lag from the response of the system. There is a similar stabilizing oscillation characteristic to the previous case in Figure 20. In Figure 21, current is the top signal, and the duty cycles are shown underneath. Once again, 
the oscillation becomes minimized when the derivative element is applied. The battery's initial SoC is $50 \%$. Extra test cases are in B.3.

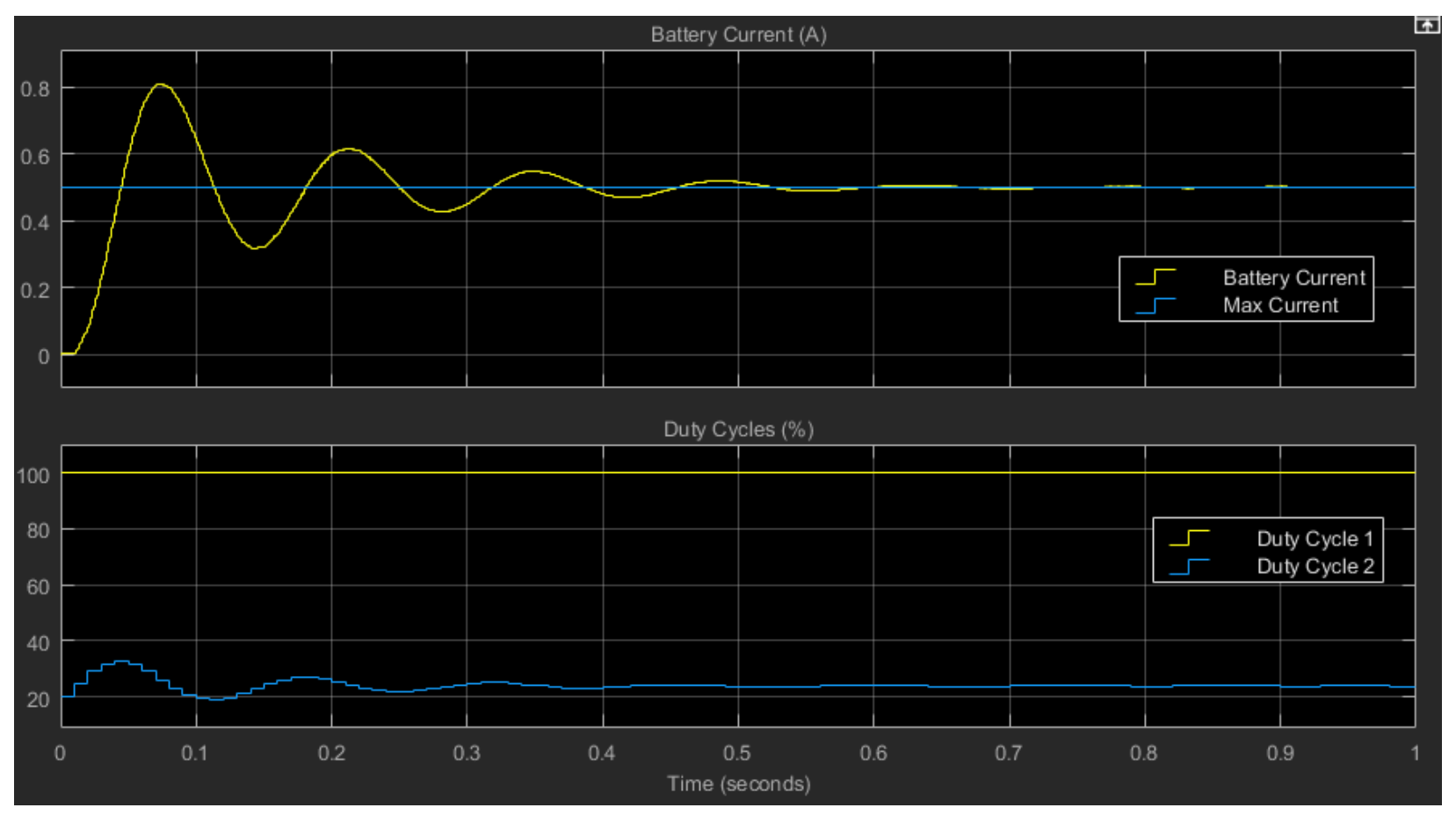

Figure 21. Example Case: $e=0.05, f=0$

\subsection{Parameter Selection}

This section contains the selected parameters for equations 1, 2, and 3 under the maximum rated values given in section 4.2.

\subsubsection{MPPT Control Parameters}

Figure 22 shows the test case for the chosen parameters in equation 1 . The peak time is 0.11 seconds and the convergence is 0.14 seconds. The smaller magnitude of $a+$ slightly overshoots the duty cycle because of the steep slope in power-voltage curve, but the larger magnitude of $a$ - quickly converges power to MPP from the shallow slope side. The small P\&O maintains maximum power and allows the system to track quickly if the power changes. 


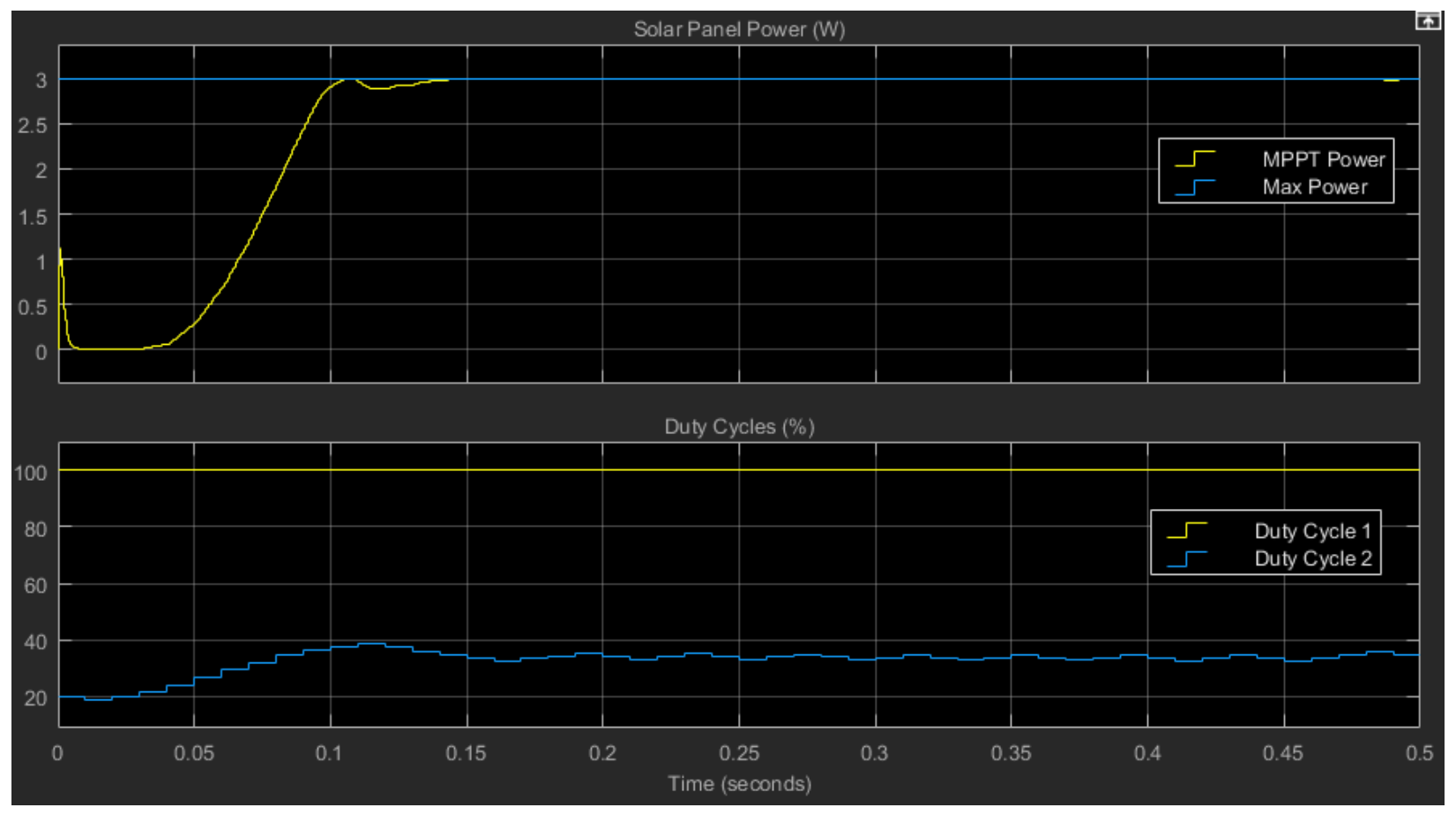

Figure 22. Selected Case: $a+=-0.001, a-=-0.005,|b|=0.01$

\subsubsection{Battery Voltage Control Parameters}

Figure 23 displays the convergence case for the chosen parameters in equation 2 . The peak time is 0.15 seconds and the convergence time is less than 0.3 seconds. The large magnitude of $c$ gives quick peak times and the magnitude of $d$ determines the convergence rate. If $d$ is too small, there is an overshoot because of $c$. If $d$ is too large, the convergence requires a significant amount of time unless $c$ is further increased; but this can cause instability with such significant step sizes. The slight oscillation is due to limited variability of the PWM. 


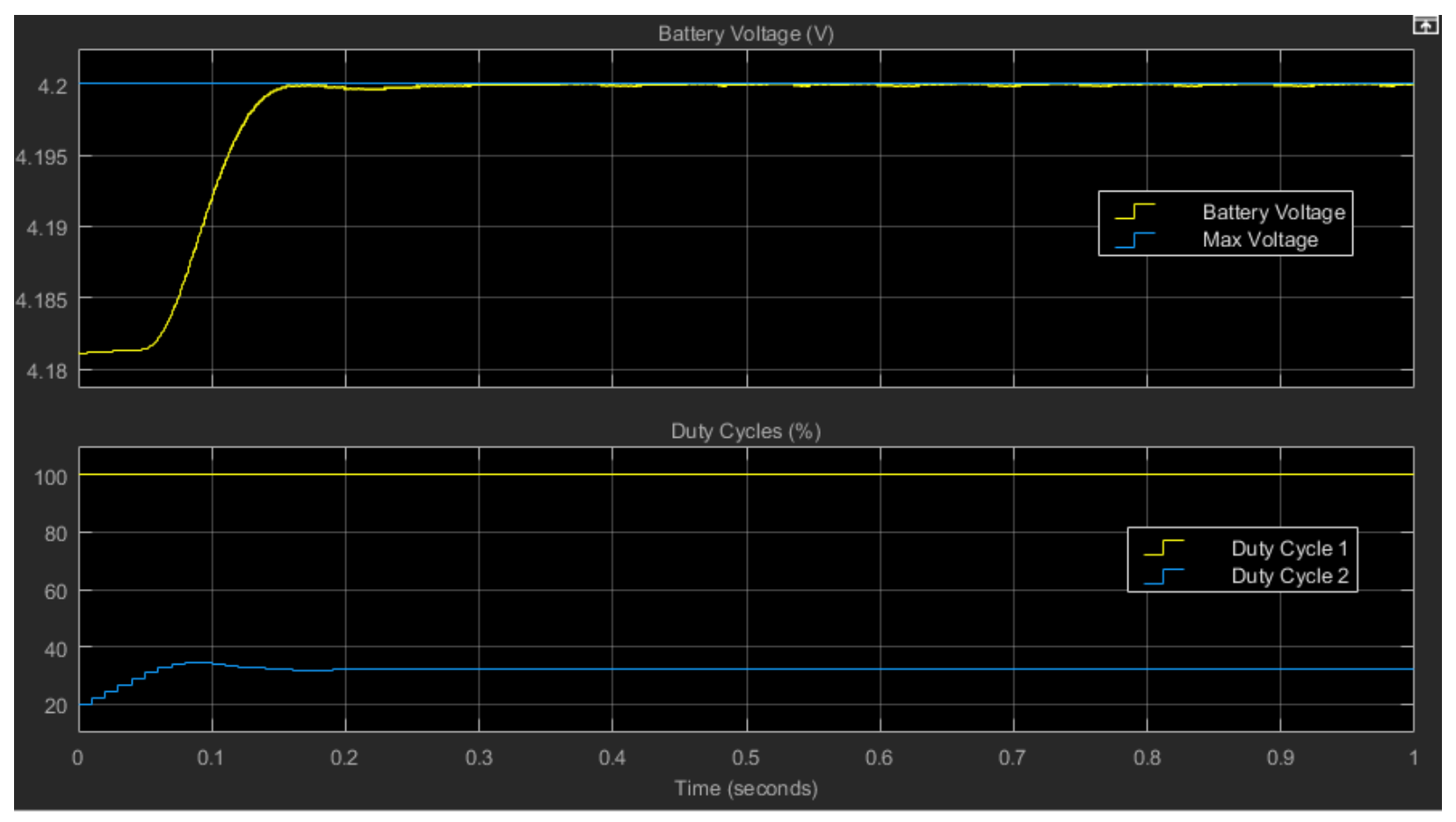

Figure 23. Selected Case: $c=5, d=-4.5$

\subsubsection{Battery Current Control Parameters}

Figure 24 represents the convergence case for the chosen parameters in equation 3 . The peak time and convergence time are both 0.11 seconds. Higher values of $e$ cause quicker peak times. Appropriate values of $f$ completely nullify the oscillations. Similarly as the voltage parameters, the current parameters are scaled accordingly together for stability purposes. 


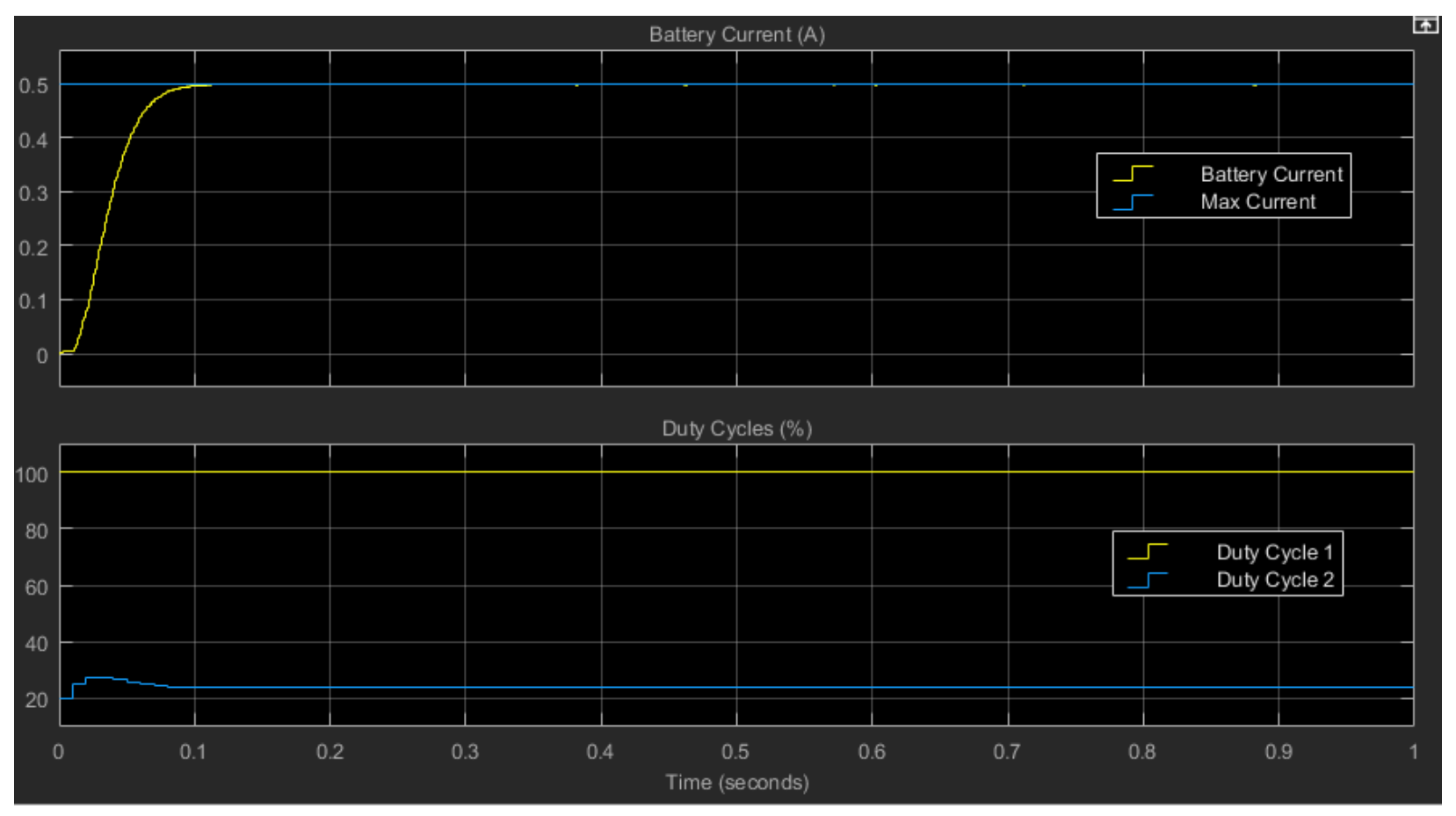

Figure 24. Selected Case: $e=0.05, f=-0.25$

\subsection{Algorithm Comparison}

Table 1 shows the results from the adaptive incremental conductance method, $\mathrm{P} \& \mathrm{O}$ method, and proposed hybrid method. The adaptive incremental conductance cases are when parameter $b$ is set to zero, the $\mathrm{P} \& \mathrm{O}$ cases are when parameter $a$ is set to zero, and the hybrid cases are when neither $a$ nor $b$ are set to zero. 
Table 1. Performance Results of MPPT Methods

\begin{tabular}{|c|c|c|c|c|c|c|}
\hline Method & $a(a+, a-)$ & $|b|$ & $\begin{array}{c}\text { Peak } \\
\text { Time } \\
(\mathrm{s})\end{array}$ & $\begin{array}{c}\text { Power } \\
\text { Undershoot } \\
(\%)\end{array}$ & $\begin{array}{c}\text { Convergence } \\
\text { Time } \\
(\mathrm{s})\end{array}$ & $\begin{array}{c}\text { Steady-State } \\
\text { Error }(\%)\end{array}$ \\
\hline Inc. Con. 1 [12] & -0.005 & 0 & 0.09 & 14 & 0.475 & 0 \\
\hline Inc. Con. 2 [12] & -0.01 & 0 & 0.06 & 28 & 0.4 & 0 \\
\hline Inc. Con. 3 [12] & -0.02 & 0 & 0.05 & 64 & 0.325 & 0 \\
\hline Inc. Con. 4 [12] & $-0.0025,-0.02$ & 0 & 0.36 & 6 & 0.41 & 0 \\
\hline Inc. Con. 5 [12] & $-0.005,-0.05$ & 0 & 0.09 & 13 & 0.14 & 0 \\
\hline P\&O 1 [8] & 0 & 0.005 & 0.29 & 0 & 0.29 & 0 \\
\hline P\&O 2 [8] & 0 & 0.01 & 0.17 & 0 & 0.17 & 0 \\
\hline P\&O 3 [8] & 0 & 0.02 & 0.12 & 2 & 0.17 & 0.5 \\
\hline P\&O 4 [8] & 0 & 0.035 & 0.085 & 8.3 & 0.125 & 2 \\
\hline P\&O 5 [8] & 0 & 0.05 & 0.075 & 16.7 & 0.12 & 6 \\
\hline Hybrid 1 & $-0.001,-0.005$ & 0.01 & 0.11 & 4 & 0.14 & 0 \\
\hline Hybrid 2 & -0.0025 & 0.01 & 0.08 & 8.3 & 0.16 & 0 \\
\hline Hybrid 3 & $-0.0025,-0.01$ & 0.01 & 0.08 & 8.3 & 0.14 & 0 \\
\hline Hybrid 4 & -0.005 & 0.01 & 0.06 & 16.7 & 0.18 & 0.5 \\
\hline Hybrid 5 & $-0.005,-0.05$ & 0.01 & 0.06 & 16.7 & 0.11 & 1 \\
\hline
\end{tabular}

Hybrid 1 is the selected parameter case for the MPPT algorithm and, thus, is for the comparison. Inc. Con. 5 and $\mathrm{P} \& \mathrm{O} 2$ are selected to compare with Hybrid 1. Figure 25 represents the comparison plot of the three selected methods and can be used for visual reference. The hybrid method has a peak time $20 \mathrm{~ms}$ slower than adaptive incremental conductance and $60 \mathrm{~ms}$ faster than P\&O. The hybrid method has a power undershoot that's 9\% less in magnitude than adaptive incremental conductance and $4 \%$ more in magnitude than P\&O. The hybrid method has the same convergence time as adaptive incremental conductance and has a $30 \mathrm{~ms}$ faster convergence than $\mathrm{P} \& \mathrm{O}$. All three cases share the same steady-state error of $0 \%$. Over all cases, the hybrid method minimizes the steady-state error of $\mathrm{P} \& \mathrm{O}$, and maximizes the convergence rate of adaptive incremental conductance. In summary, the hybrid method provides more robust results, but the complexity is higher than using one of the single methods. 
Method: Inc. Con. 5 [12]

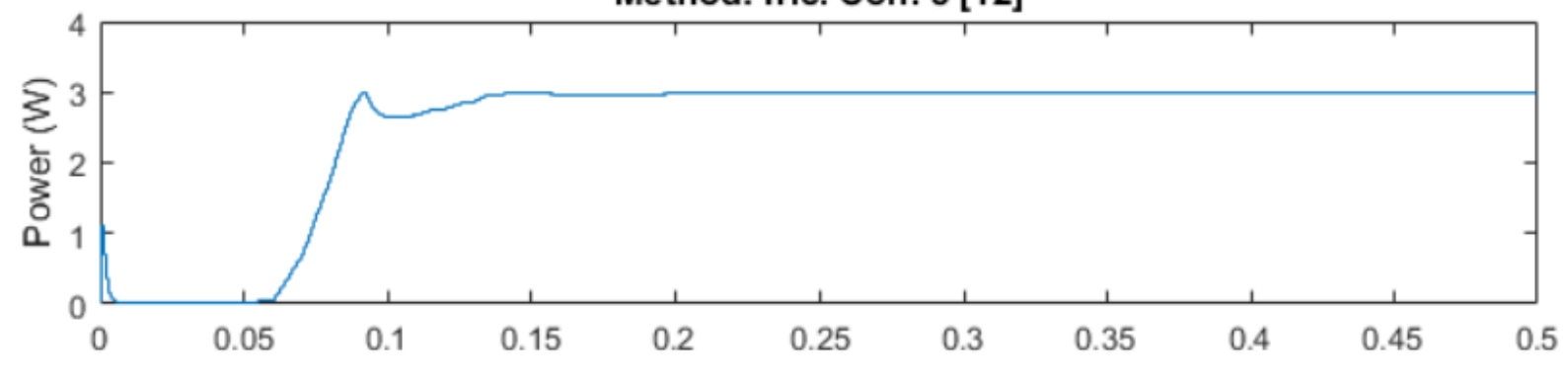

Method: P\&O 2 [8]

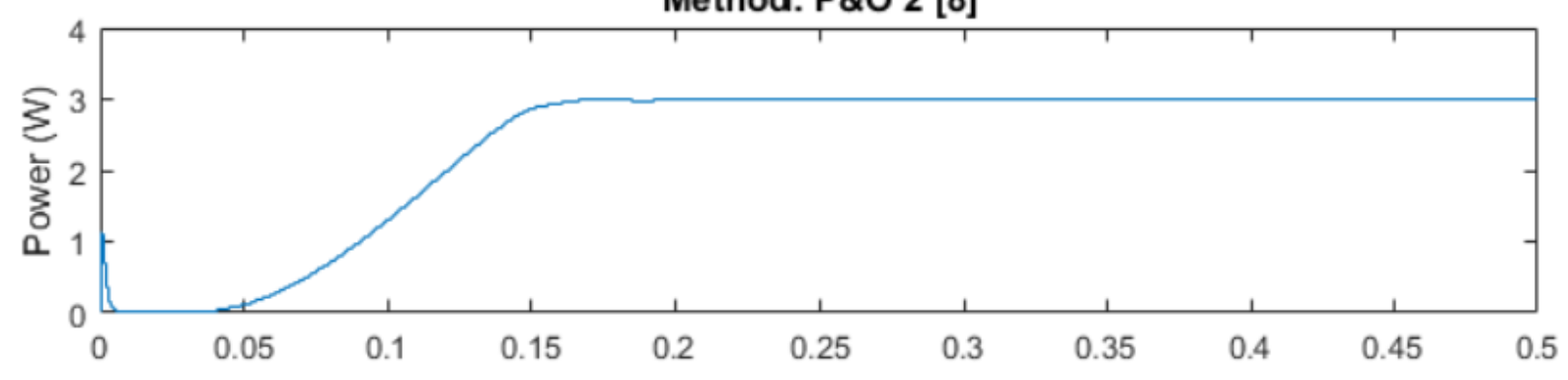

Method: Hybrid 1

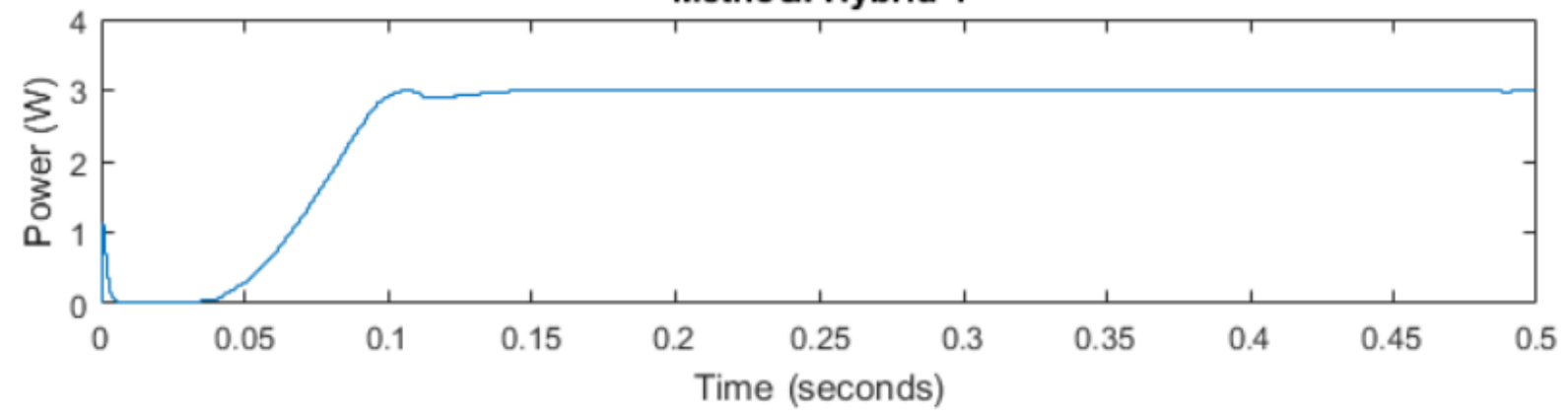

Figure 25. Comparison Plot for the Three Compared Methods 


\section{Chapter 5. Conclusion and Future Work}

In this chapter the control system and models are concluded and the possible future work for the control system and models are presented.

\subsection{Conclusion}

The control system MPP tracks the solar panel and limits the output voltage of the power converter to the maximum rated voltage of the battery. Though the algorithm was developed for current limiting too, equation 3 is unnecessary for joint simulations since the solar panel used doesn't deliver enough power in any case to push the battery past a true maximum rated current, unlike the mere $0.5 \mathrm{~A}$ chosen for completeness. The hybrid MPPT method converges significantly faster to MPP than the traditional adaptive incremental conductance. This happens because, initially, adaptive incremental conductance might not work properly since $\mathrm{dP} / \mathrm{dV}$ is a complicated calculation from the resting point, thus, the initial $\mathrm{P} \& \mathrm{O}$ can activate the $\mathrm{dP} / \mathrm{dV}$ term.

Figure 26 shows a two second simulation using both equation 1 and equation 2 . Initially the duty step is calculated in equation 1 to converge to max power until the battery voltage gets reasonably close to max; then equation 2 calculates the new duty steps. As the battery begins to fully charge, the power from the solar panel begins to reduce slightly but noticeably, as seen in the top graph. P\&O method dominates first (small slope start), then adaptive incremental conductance (high slope phase), followed with the convergence to max battery voltage (small slope end). It is clear from the plot that this is a $\mathrm{CP} / \mathrm{CV}$ charge technique as wanted. Therefore, the overall control system is successful. 


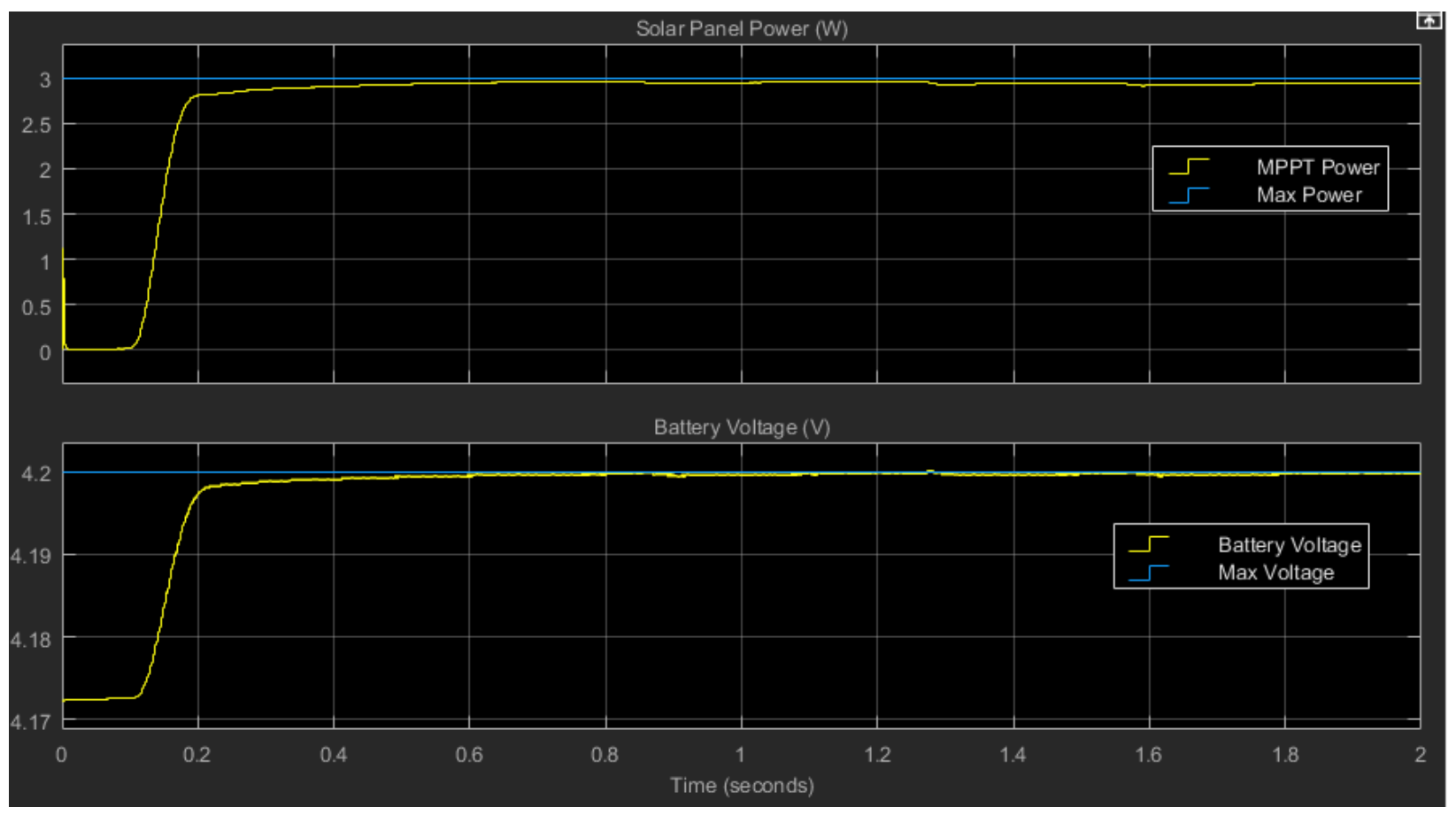

Figure 26. Example of Control System MPPT and Voltage Limiting

Though the control algorithm operates appropriately, there are a couple drawbacks to consider with this system: the algorithm is designed to a specific P-V curve, the voltage slightly exceeds its maximum (put $\mathrm{V}_{\max }$ less than battery's max rated voltage in equation 2 to fix), and the decent overshoot in duty cycle on converging to the MPP with equation 1 . Another issue faced was the time for longer simulations; running the entire system at $5 \mathrm{MHz}$ was the prime issue for this, but in return offered more accurate simulation results.

\subsection{Future Work}

There are still many possible future opportunities for advancing this algorithm and improving its robustness. Instead of using a constant irradiance of $100 \%$, a varied irradiance can assist choosing the correct equation parameters for MPPT. The solar panel model can expand to a 2D lookup table, 3D lookup table (voltage, irradiance, and temperature dependence), or even 
an equation. The power requested may change throughout the simulation rather than the constant OW in these simulations; though this probably has minimal effect on equation parameters. Another possible improvement is PID control for equation 2 and 3 as opposed to the selected control method. A huge upgrade to the algorithm is a method to automatically update the parameters for any solar panel with the same power converter under certain peak time and convergence time conditions.

Varying the circuit components or swapping with non-linear circuit components for the power converter can drastically change every aspect of its characteristics; thus, this is a very interesting area to investigate. Replacing the MOSFETs with current FETs is sought to further improve the power converter. The battery's series resistance in the power converter model is assumed to remain constant over its charge, however, this can be a variable parameter based on SoC. Also, a way to sync the two duty cycles with the power converter such that the buck control isn't always $100 \%$ when boosting or the boost control isn't always $0 \%$ when bucking; therefore, creating a potentially more configurable algorithm. However, the chosen power converter topology does not adequately operate the solar panel at MPP when it is in buck mode, so different power converters, such as a boost-buck converter, should be explored. 


\section{REFERENCES}

[1] Ahmad, Jawad. "A Fractional Open Circuit Voltage Based Maximum Power Point Tracker for Photovoltaic Arrays." 2010 2nd International Conference on Software Technology and Engineering (2010): n. pag. Web.

[2] Alajmi, Bader N., Khaled H. Ahmed, Stephen J. Finney, and Barry W. Williams. "FuzzyLogic-Control Approach of a Modified Hill-Climbing Method for Maximum Power Point in Microgrid Standalone Photovoltaic System." IEEE Transactions on Power Electronics 26.4 (2011): 1022-030. Web.

[3] Armstrong, S., M.e. Glavin, and W.g. Hurley. "Comparison of Battery Charging Algorithms for Stand Alone Photovoltaic Systems." 2008 IEEE Power Electronics Specialists Conference (2008): n. pag. Web.

[4] Baimel, D., R. Shkoury, L. Elbaz, S. Tapuchi, and N. Baimel. "Novel Optimized Method for Maximum Power Point Tracking in PV Systems Using Fractional Open Circuit Voltage Technique." 2016 International Symposium on Power Electronics, Electrical Drives, Automation and Motion (SPEEDAM) (2016): n. pag. Web.

[5] Biabani, Mohammed Abdul Khader Aziz, and Fazeel Ahmed. "Maximum Power Point Tracking of Photovoltaic Panels Using Perturbation and Observation Method and Fuzzy 
Logic Control Based Method." 2016 International Conference on Electrical, Electronics, and Optimization Techniques (ICEEOT) (2016): n. pag. Web.

[6] Biswas, Suvankar, Lilly Huang, Vaibhav Vaidya, Krishnan Ravichandran, Ned Mohan, and Sairaj V. Dhople. "Universal Current-Mode Control Schemes to Charge Li-Ion Batteries Under DC/PV Source." IEEE Transactions on Circuits and Systems I: Regular Papers 63.9 (2016): 1531-542. Web

[7] Elgendy, Mohammed A., Bashar Zahawi, and David J. Atkinson. "Operating Characteristics of the P\&O Algorithm at High Perturbation Frequencies for Standalone PV Systems." IEEE Transactions on Energy Conversion 30.1 (2015): 189-98. Web.

[8] Esram, Trishan, and Patrick L. Chapman. "Comparison of Photovoltaic Array Maximum Power Point Tracking Techniques." IEEE Transactions on Energy Conversion 22.2 (2007): 439-49. Web.

[9] Gibson, T.1., and N.a. Kelly. "Solar Photovoltaic Charging of Lithium-ion Batteries." 2009 IEEE Vehicle Power and Propulsion Conference (2009): n. pag. Web.

[10] Gil-Antonio, Leopoldo, Martha Belem Saldivar-Marquez, and Otniel Portillo-Rodriguez. "Maximum Power Point Tracking Techniques in Photovoltaic Systems: A Brief Review." 2016 13th International Conference on Power Electronics (CIEP) (2016): n. pag. Web. 
[11] Jana, Joydip, Konika Das Bhattacharya, and Hiranmay Saha. "Design \& Implementation of MPPT Algorithm for Battery Charging with Photovoltaic Panel Using FPGA." 2014 6th IEEE Power India International Conference (PIICON) (2014): n. pag. Web.

[12] Li, Chendi, Yuanrui Chen, Dongbao Zhou, Junfeng Liu, and Jun Zeng. "A HighPerformance Adaptive Incremental Conductance MPPT Algorithm for Photovoltaic Systems." Energies 9.4 (2016): 288. Web.

[13] Padhee, Subhransu, Umesh Chandra Pati, and Kamalakanta Mahapatra. "Design of Photovoltaic MPPT Based Charger for Lead-acid Batteries." 2016 IEEE International Conference on Emerging Technologies and Innovative Business Practices for the Transformation of Societies (EmergiTech) (2016): n. pag. Web.

[14] Palmiro, Flavio, Ramesh Rayudu, and Rebecca Ford. "Modelling and Simulation of a Solar PV Lithium Ion Battery Charger for Energy Kiosks Application." 2015 IEEE PES AsiaPacific Power and Energy Engineering Conference (APPEEC) (2015): n. pag. Web.

[15] Pastre, Marc, Francois Krummenacher, Onur Kazanc, Naser Khosro Pour, Catherine Pace, Stefan Rigert, and Maher Kayal. "A Solar Battery Charger with Maximum Power Point Tracking." 2011 18th IEEE International Conference on Electronics, Circuits, and Systems (2011): n. pag. Web. 
[16] Petchjatuporn, Panom, Wannaya Ngamkham, Noppadol Khaehintung, Phaophak Sirisuk, Wiwat Kiranon, and Anatawat Kunakorn. "A Solar-powered Battery Charger with Neural Network Maximum Power Point Tracking Implemented on a Low-Cost PICmicrocontroller." TENCON 2005 - 2005 IEEE Region 10 Conference (2005): n. pag. Web.

[17] Purnama, Irwan, Yu-Kang Lo, and Huang-Jen Chiu. "A Fuzzy Control Maximum Power Point Tracking Photovoltaic System." 2011 IEEE International Conference on Fuzzy Systems (FUZZ-IEEE 2011) (2011): n. pag. Web.

[18] Shen, Weixiang, Thanh Tu Vo, and Ajay Kapoor. "Charging Algorithms of Lithium-ion Batteries: An Overview." 2012 7th IEEE Conference on Industrial Electronics and Applications (ICIEA) (2012): n. pag. Web.

[19] Sitbon, M., S. Gadelovits, and A. Kuperman. "Multi-output Portable Solar Charger for LiIon Batteries." 7th IET International Conference on Power Electronics, Machines and Drives (PEMD 2014) (2014): n. pag. Web

[20] Subudhi, Bidyadhar, and Raseswari Pradhan. "A Comparative Study on Maximum Power Point Tracking Techniques for Photovoltaic Power Systems." IEEE Transactions on Sustainable Energy 4.1 (2013): 89-98. Web. 
[21] Tahiri, F.e., K. Chikh, M. Khafallah, and A. Saad. "Comparative Study between Two Maximum Power Point Tracking Techniques for Photovoltaic System." 2016 International Conference on Electrical and Information Technologies (ICEIT) (2016): n. pag. Web.

[22] Vo, Thanh Tu, Weixiang Shen, and Ajay Kapoor. "Experimental Comparison of Charging Algorithms for a Lithium-ion Battery." 2012 10th International Power \& Energy Conference (IPEC) (2012): n. pag. Web. 


\section{APPENDICES}

\section{Appendix A. Simulink Models of the Control System}

This appendix consists of the Simulink models for the entire system in Figure 1. First, there are the physical component models and the sample source code for the control algorithm. Second, there is the updated Simulink model for the control system.

\section{A.1. Simulink Model of Entire System}

Figure 27 is a simulink representation of the entire physical system. The measuring devices are inside the respective models and involves small error measurments to enhance the total stability of the control system. The irradiance is set to 1 and the power request is set to 0 .

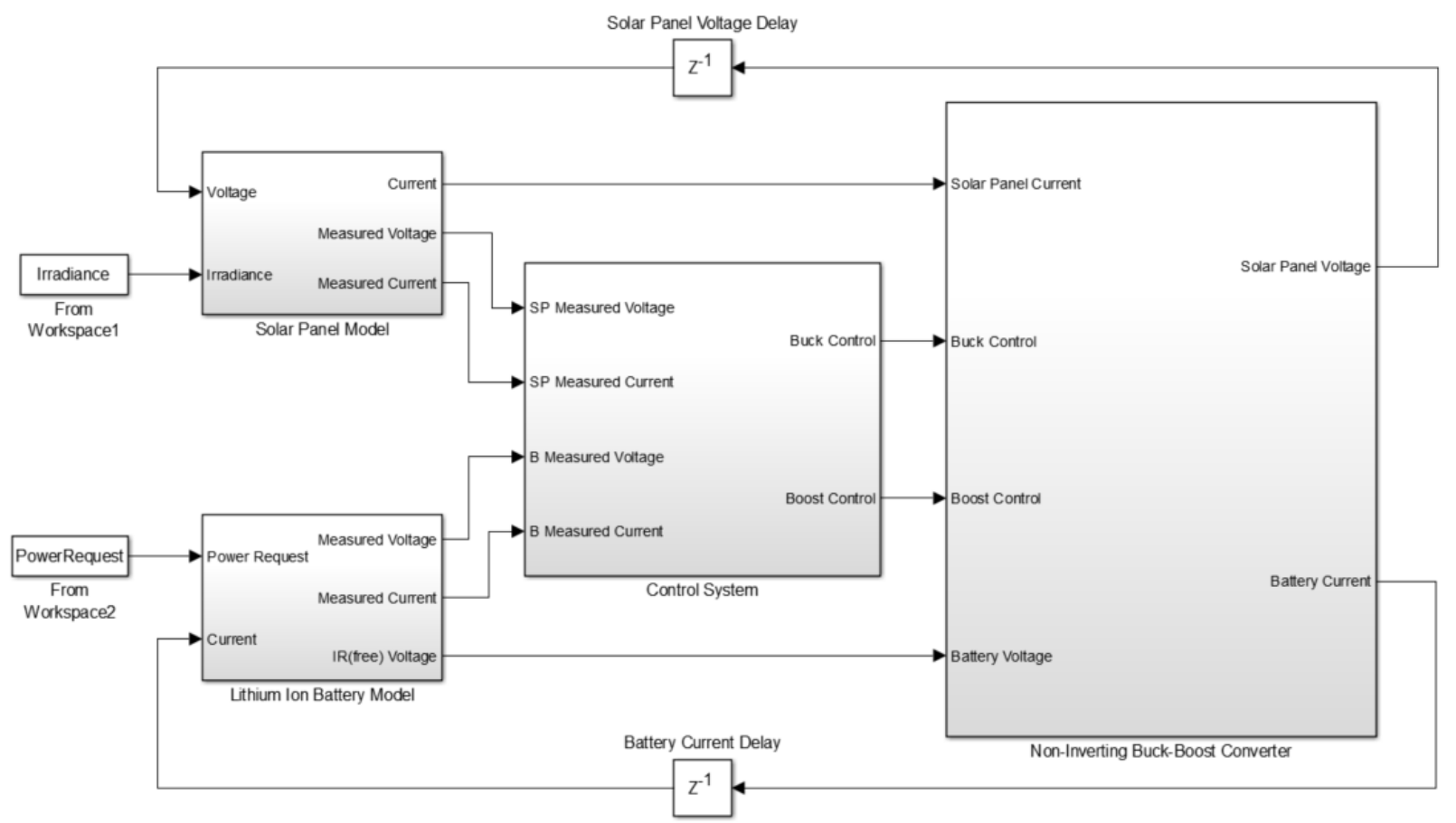

Figure 27. Simulink Model of Entire System 


\section{A.1.1. Solar Panel}

The Solar Panel Model, Figure 28, is a simple 1-D look up table that scales depending on irradiance. Perhaps it may be characterized as an electrical circuit as well as the battery, but the normal lookup table gives astoundingly enough accuracy since solar panels are easy to characterize as so. The values for the Solar Panel Voltage Lookup 1-D block are from realistic data acquired via the manufacturer, where the MPP of the solar panel is $3 \mathrm{~W}$.

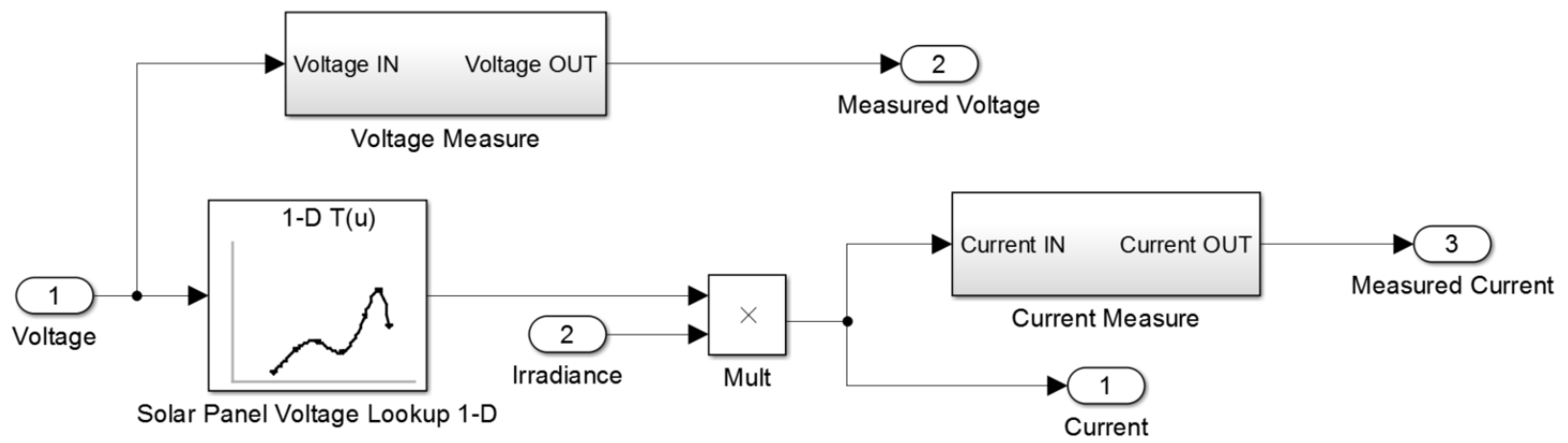

Figure 28. Simulink Model of Solar Panel

\section{A.1.2. Battery}

The high level block diagram for the battery allows power requests to interfere with the actual circuit to further enhance robustness of control. Figure 29 is the high level Simulink model of a battery. This model represents the electrical circuit for a $\mathrm{Li}+$ battery. However, there is an amp-hour integrator inside this model to know where the battery's $\mathrm{SoC}$ is at so the relative OCV, Rs, R1, C1, R2, and C2 are used. Note: V(IRfree) is the voltage of the battery minus the voltage across the series resistance. 


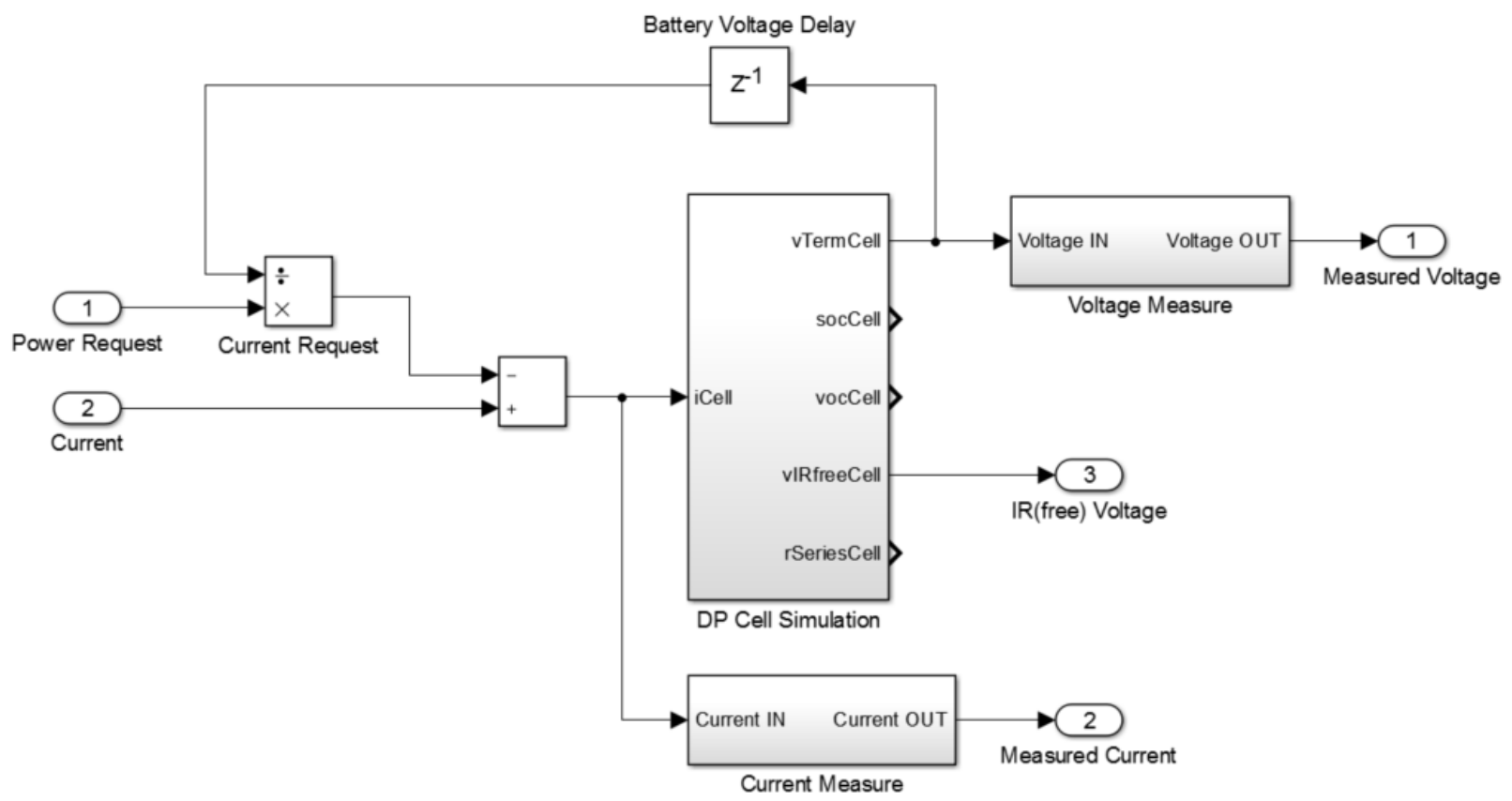

Figure 29. Simulink Model of Battery

The actual representation of the battery is given in Figure 30. There is an open circuit voltage lookup for the battery based on different state of charges. Below those blocks are the resistance lookup tables; this value is multiplied with the current to acquire the voltage across this internal resistance. Finally, there are two more blocks that represent the transfer function for each parallel resistor and capacitor set; these are fully analyzed in the latter images. All look up tables in this block contain the realistic values, via the extraction method, for the DP model. The Capacity [Ah] block uses the battery's measured amp-hours. 


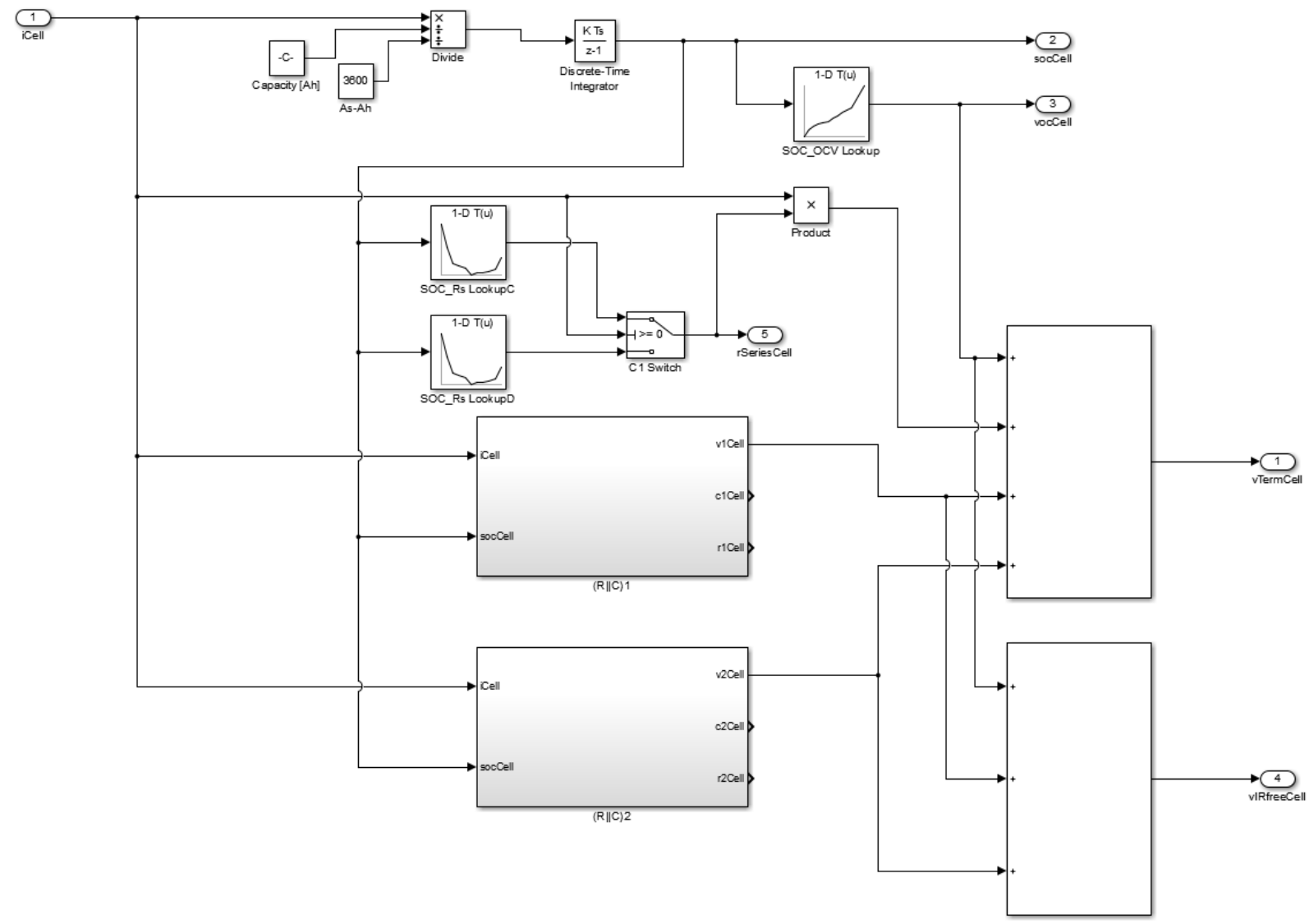

Figure 30. Simulink Model of the Li+ Battery Circuit

Figure 31 is the expanded model for a single parallel resistance and capacitor circuit; this is the representation of the transfer function in terms of Simulink blocks. Multiple look up tables are used depending on the direction of current and state of charge; and they contain the extracted values for the DP model. 


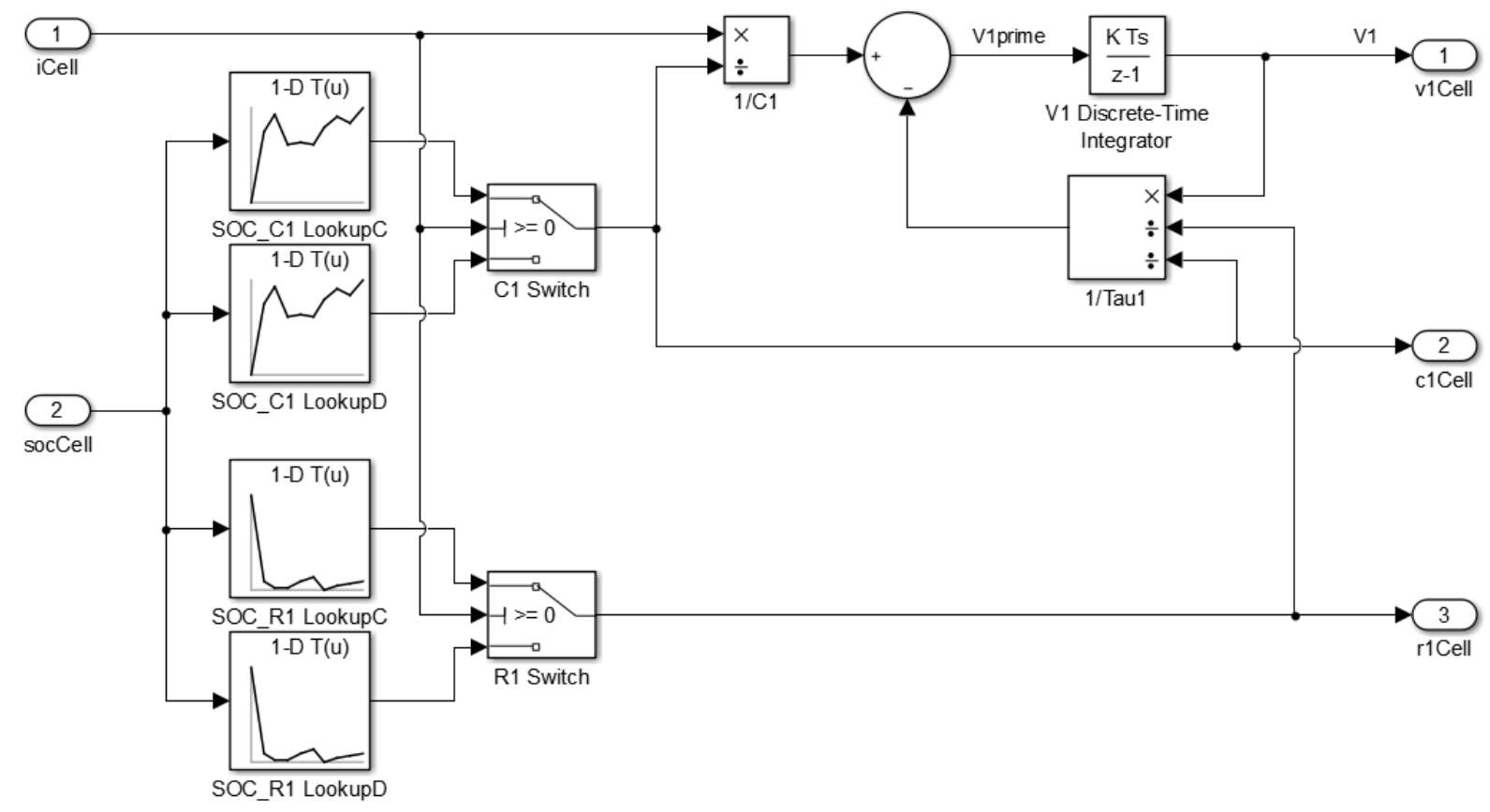

Figure 31. Simulink Model of RC Parallel Circuit

\section{A.1.3. Converter}

A Simulink model of the power electronic circuit in section 3.3 is displayed below in Figure 32. The solar panel acts like a current source and the battery acts like a voltage source with a series resistance. After the switches begin getting activated and the first set of current values are measured, the voltage and current measurements return to the solar panel and battery models in order to update the parameters once again in the circuit. The circuit components are selected to reflect the possible values for such a converter. The two capacitor and resistor series are $0.01 \mathrm{mF}$ and $0.01 \Omega$, respectively. The inductor is $20 \mathrm{mH}$. The diodes have forward voltages of $0.7 \mathrm{~V}$, and the FETs have Ron resistance of $0.1 \Omega$. The circuit operates at $5 \mathrm{MHz}$. 


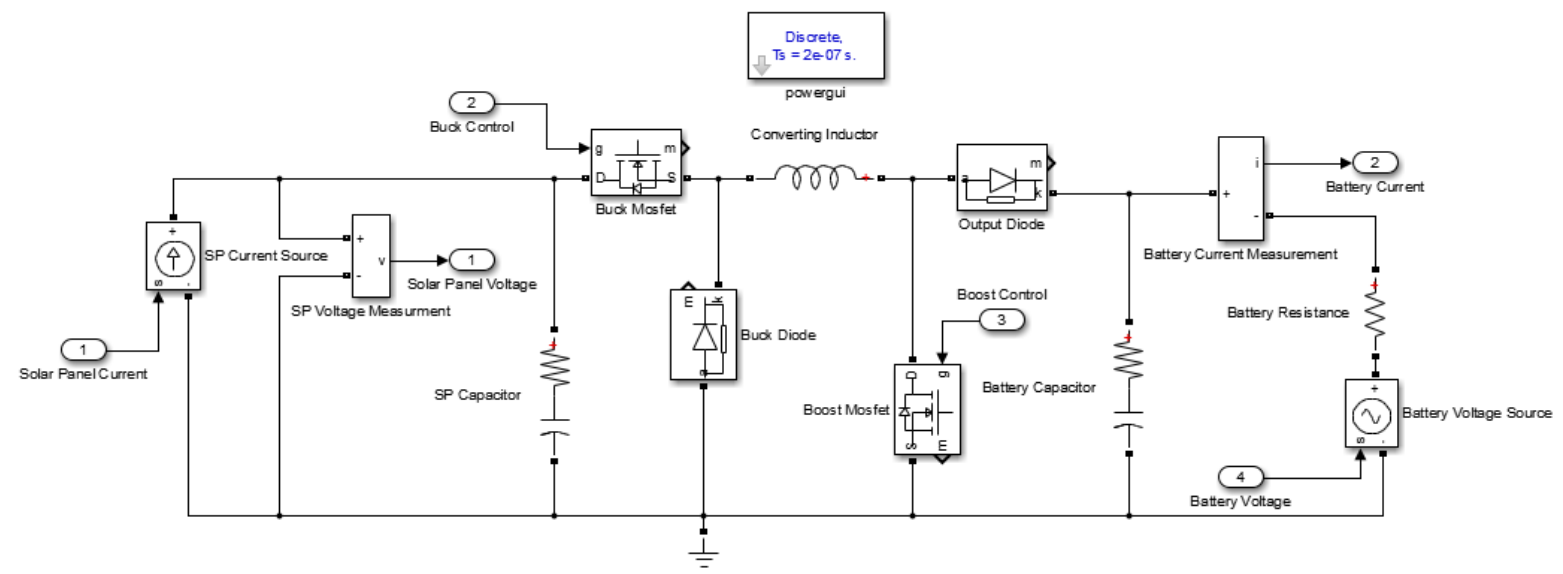

Figure 32. Simulink Model of Power Converter

\section{A.1.4. Initial Controller Design}

In Figure 33, the proof-of-operation control system is a MATLAB Function block; though this, it changes into a complex Simulink model of a MPPT and battery charging control system in section A.2. This function block does a P\&O method. This control system controls the switches on the power electronic circuit in order to determine the MPP on the solar panel and the proper amount to charge the battery at.

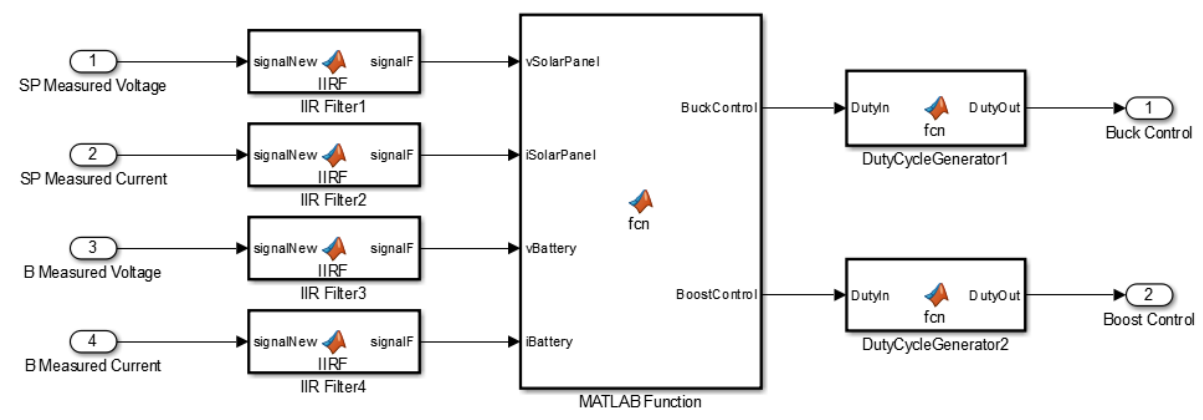

Figure 33. Preliminary Simulink Model of Control System

Here is some example code in Figure 34; it gives a general understanding of how the control system operates. Basically, the code steps around the MPP so if it happens to change, the 
control system can begin tracking it to a more ideal point. It also checks the battery's states as well to ensure it is either receiving high power charge or low power charge properly.

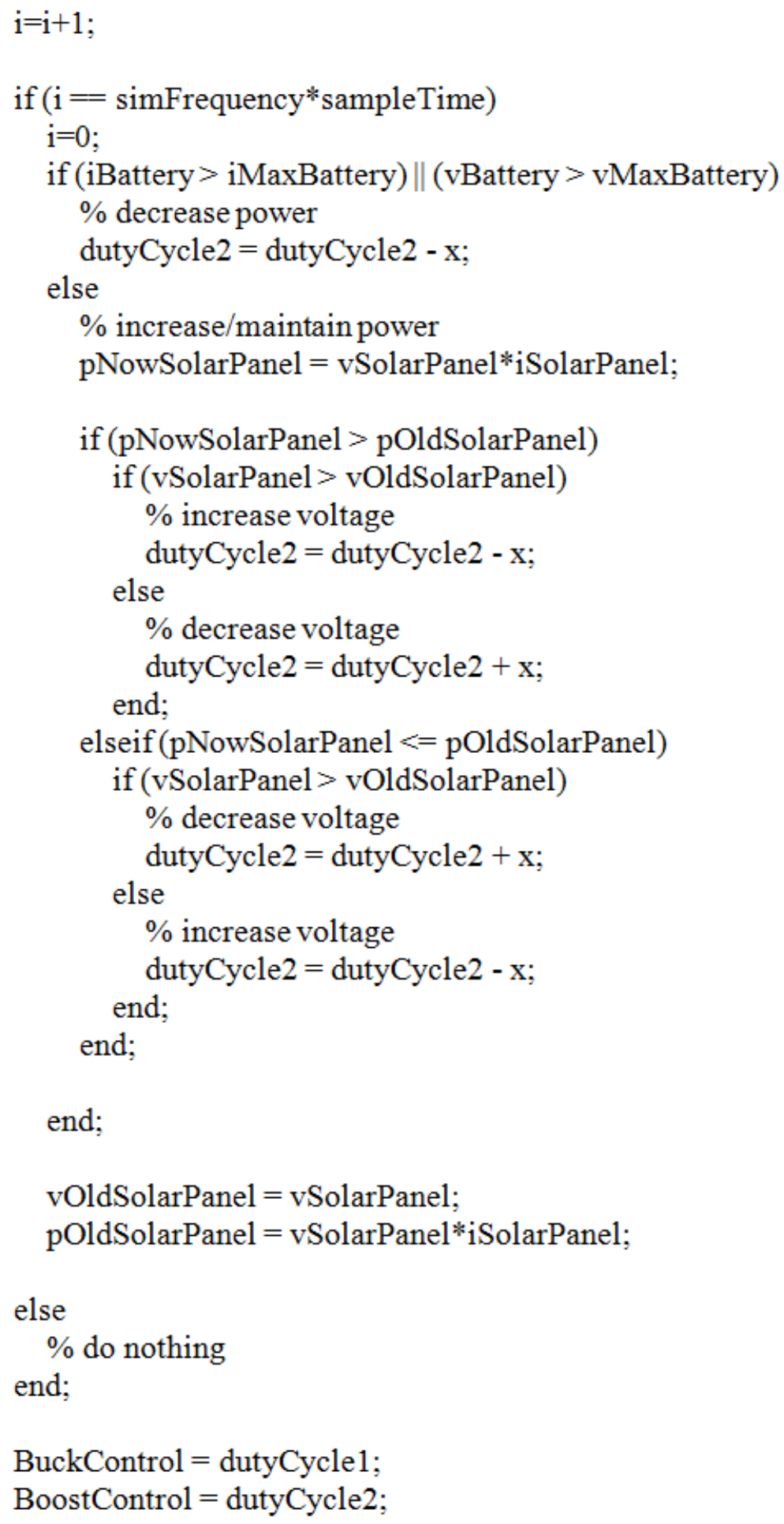

Figure 34. MATLAB Sample Code of Control Algorithm 


\section{A.2. Updated Controller Design}

With the proof-of-operation and model verification, the MATLAB Function block is swapped with a Subsystem block, as seen in Figure 35, consisting of basic Simulink blocks to enhance the speed, variability, and signal routing of the algorithm.

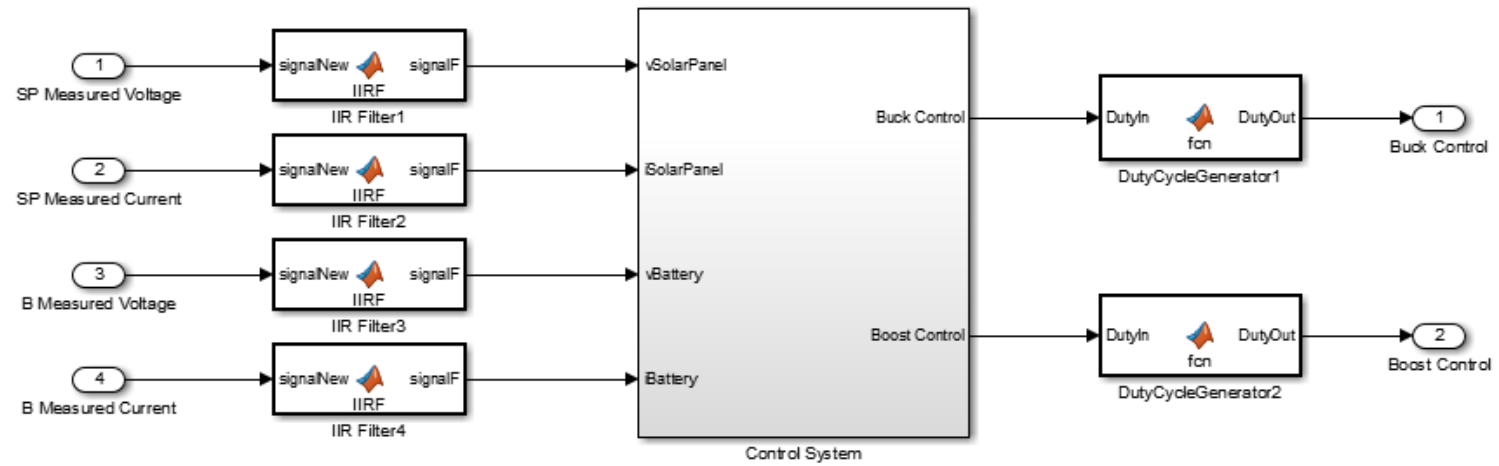

Figure 35. High Level Simulink Model of Control System

Within the Control System block, Figure 36, the algorithm is separated into five different blocks: Count, Duty Step - MPPT, Duty Step - Critical, Duty Cycle Update, and Duty Cycle Routing. The count block is used with switches to act like memory storage in-between updates; in experimentation, this block and its switch cases are removed because its sole purpose is to make the control system operate at $100 \mathrm{~Hz}$ in a $5 \mathrm{MHz}$ simulation. Some differences to notice about the new control system, is that there's a new critical duty step size correlated specifically to the battery and its limiting factors. Essentially, the updated duty cycle is the selected duty step, determined via the smallest Duty Step size, plus the original duty cycle. 


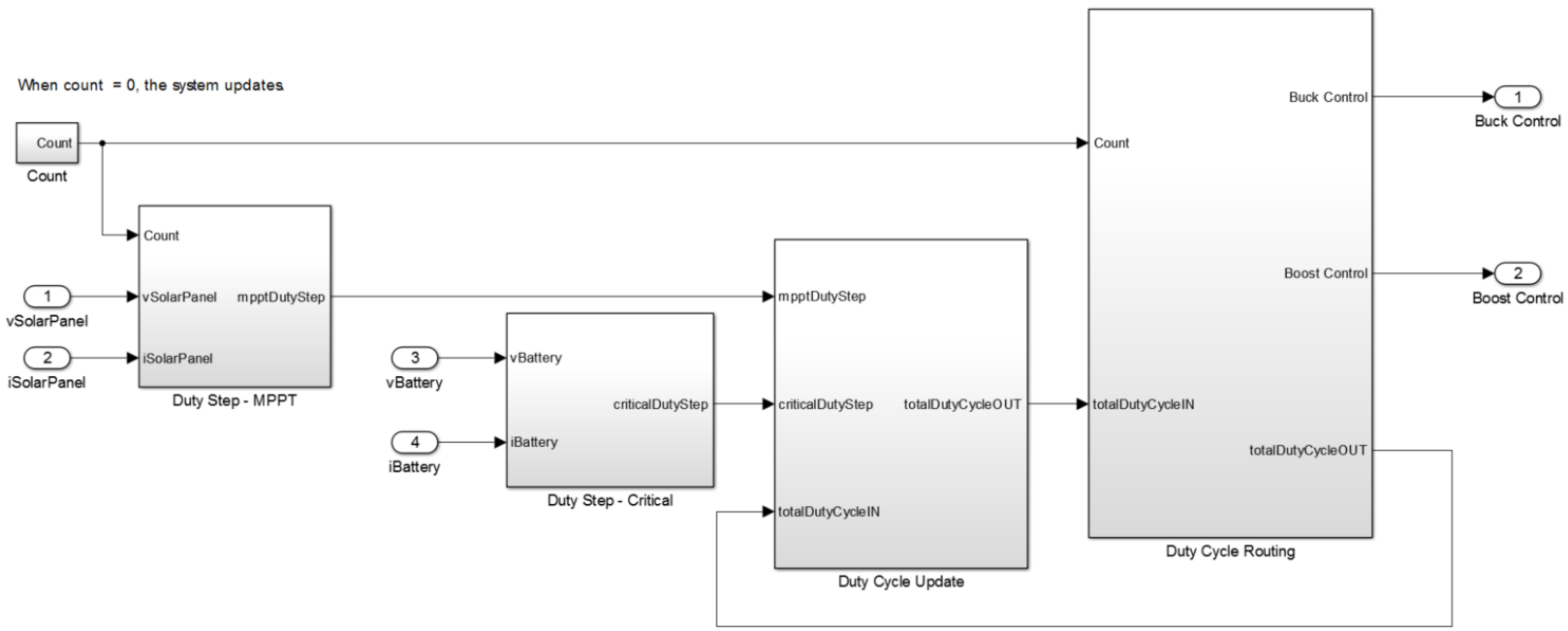

Figure 36. Low Level Simulink Model of Control System

The Count block, Figure 37, acts essentially like ' $i$ ' in the source code and is expanded below. The count is initialized at the maximum count. After every sample, the count is reduced by one. Once the count reaches zero, update cycles occur and the count is reset to the maximum count. This block is needed to properly compare and contrast values from previous update cycles.

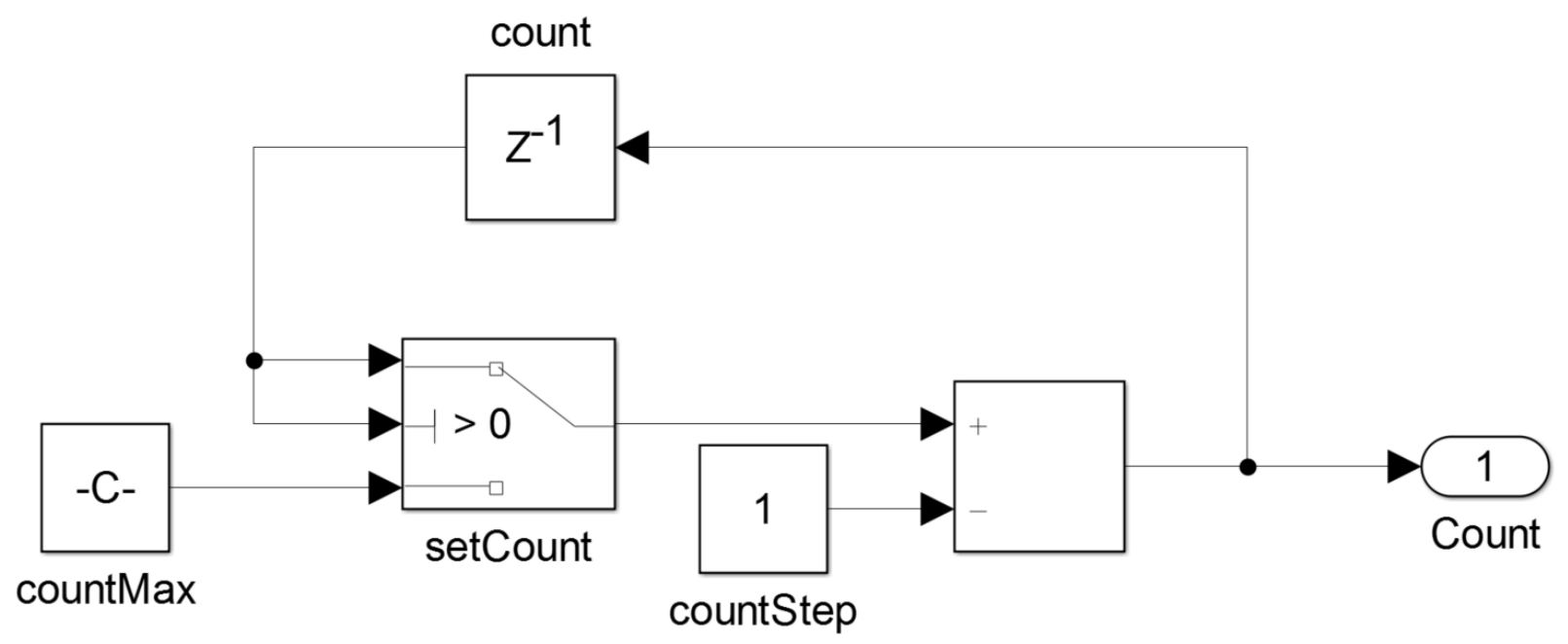

Figure 37. Simulink Model of the Count Block 


\section{A.2.1. MPPT Control Block}

The Duty Step - MPPT block, Figure 38, consist of a case selection and a selection of the proper duty cycle step. The case selection is determined between the joint relationship of the previous voltage and power to the next voltage and power. This block performs MPPT.

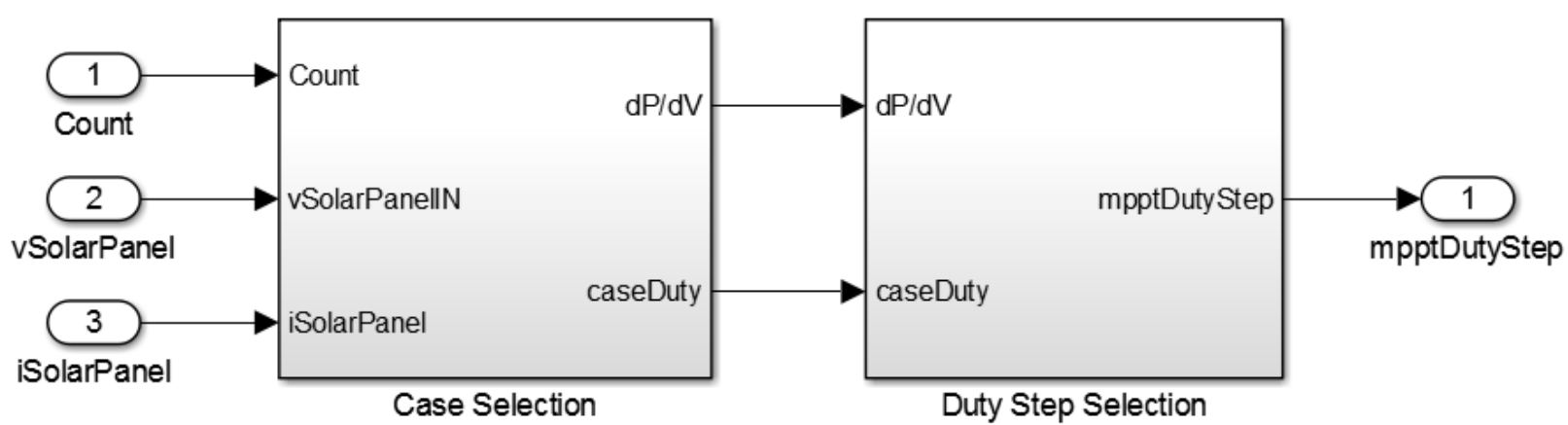

Figure 38. High Level Simulink Model of Duty Step - MPPT

The Case Selection block, Figure 39, evaluates a 'caseDuty' signal. The 'caseDuty' signal determines which direction, increase or decrease, to step the duty cycle. The value of 'caseDuty' is either 1 or -1 . A 1 is given under the condition when both the voltage and power are greater than or less than their previous values; thus, the voltage is too high and the duty step is negative to reduce the duty cycle. A -1 is given under the condition when not both voltage and power are greater than or less than their previous values; thus, the voltage is too low and the duty step is positive to increase the duty cycle. The 'count' determines when to update the feedback system (which operates like memory storage). In addition, the value of $\mathrm{dP} / \mathrm{dV}$ is also calculated for use in calculating the duty step sizes. 


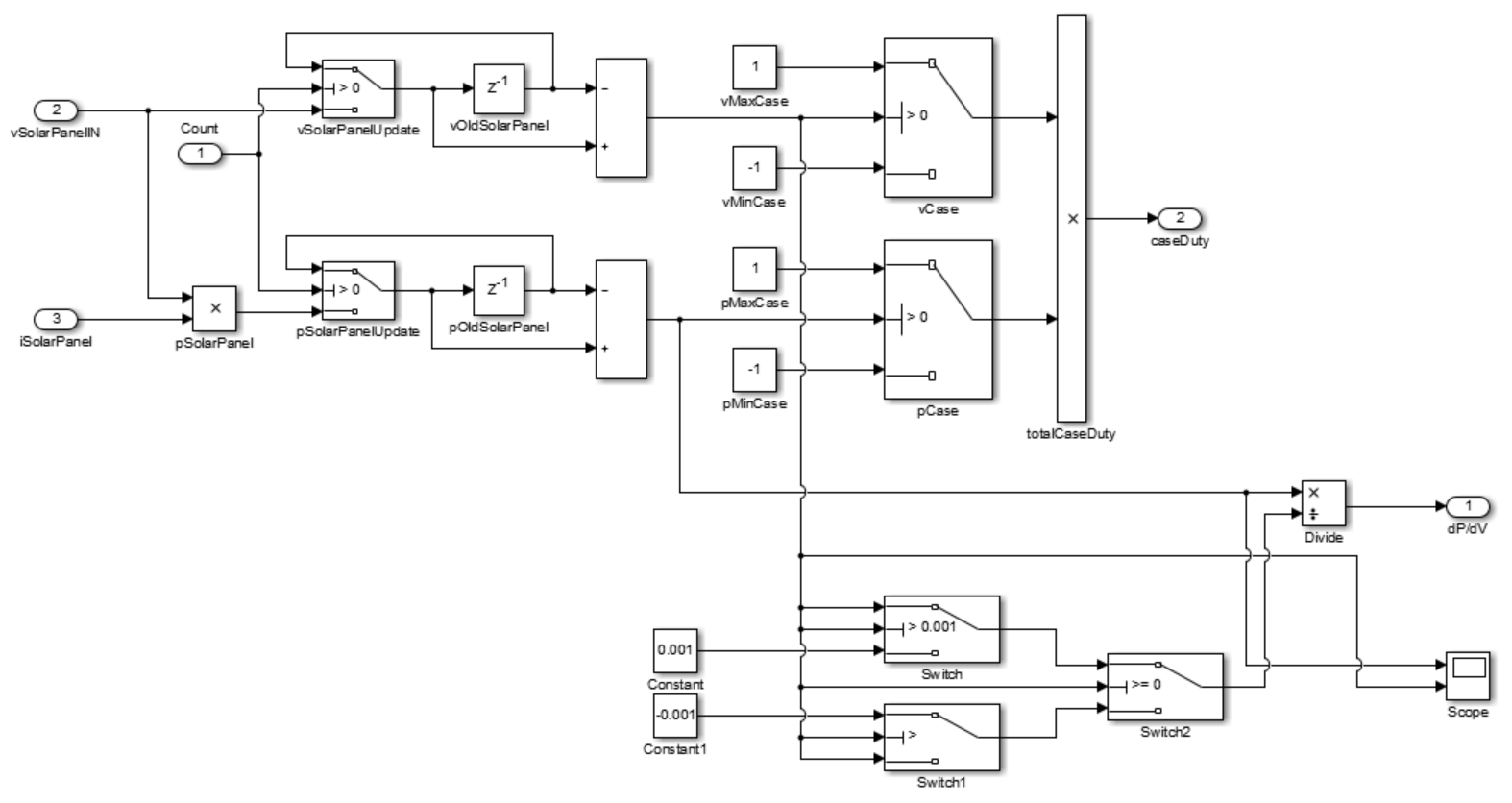

Figure 39. Simulink Model of MPPT Case Selection

The MPPT Duty Step Selection block, Figure 40, contains both the filters for the independent cases and the selected duty step for MPPT. The filters are designed such that there is a constant step up or down in conjunction with a scaled step size dependent on the value of $\mathrm{dP} / \mathrm{dV}$. They are set so the duty steps are larger when $\mathrm{dP} / \mathrm{dV}$ is large and the duty steps are smaller (but not equal to zero to avoid undefined values) for when $\mathrm{dP} / \mathrm{dV}$ are small. A faster convergence is achieved because the system has a large $\mathrm{dP} / \mathrm{dV}$ initially, and a more stable approach to the maximum power is also achieved since the step size is the minimum constant value that checks for true maximum power. The 'mpptDutyStep' is finally determined via the 'caseDuty'; gives a negative step for 'caseDuty' equal to 1 and a positive step for 'caseDuty' equal to -1 . This model represents equation 1 . 


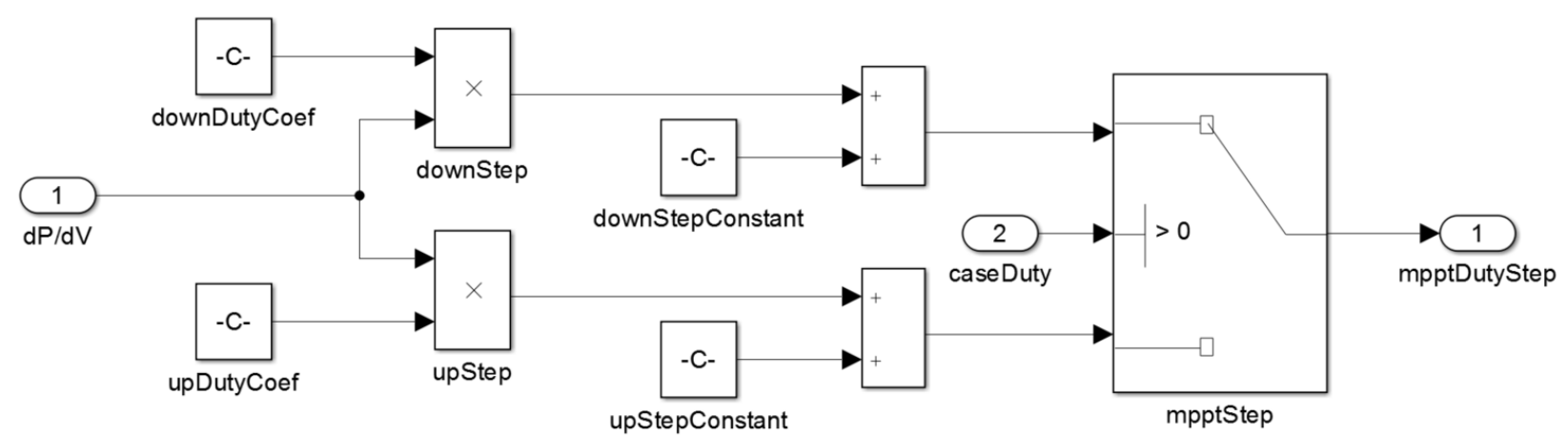

Figure 40. Simulink Model of MPPT Duty Step Selection

\section{A.2.2. Battery Limiting Control Blocks}

The Duty Step - Critical block, Figure 41, is composed of two independent duty step sizes and the selection method between the critical duty steps. To minimize the oscillations near limits, separate duty steps are created for the critical case when the maximum power of the solar panel overloads the battery. The priority of a step is based on its size, the smallest one is selected. However in practice, the critical current case step most certainly isn't used since batteries can handle well above the current outputs from the converter; though it is included for completeness of the algorithm. 


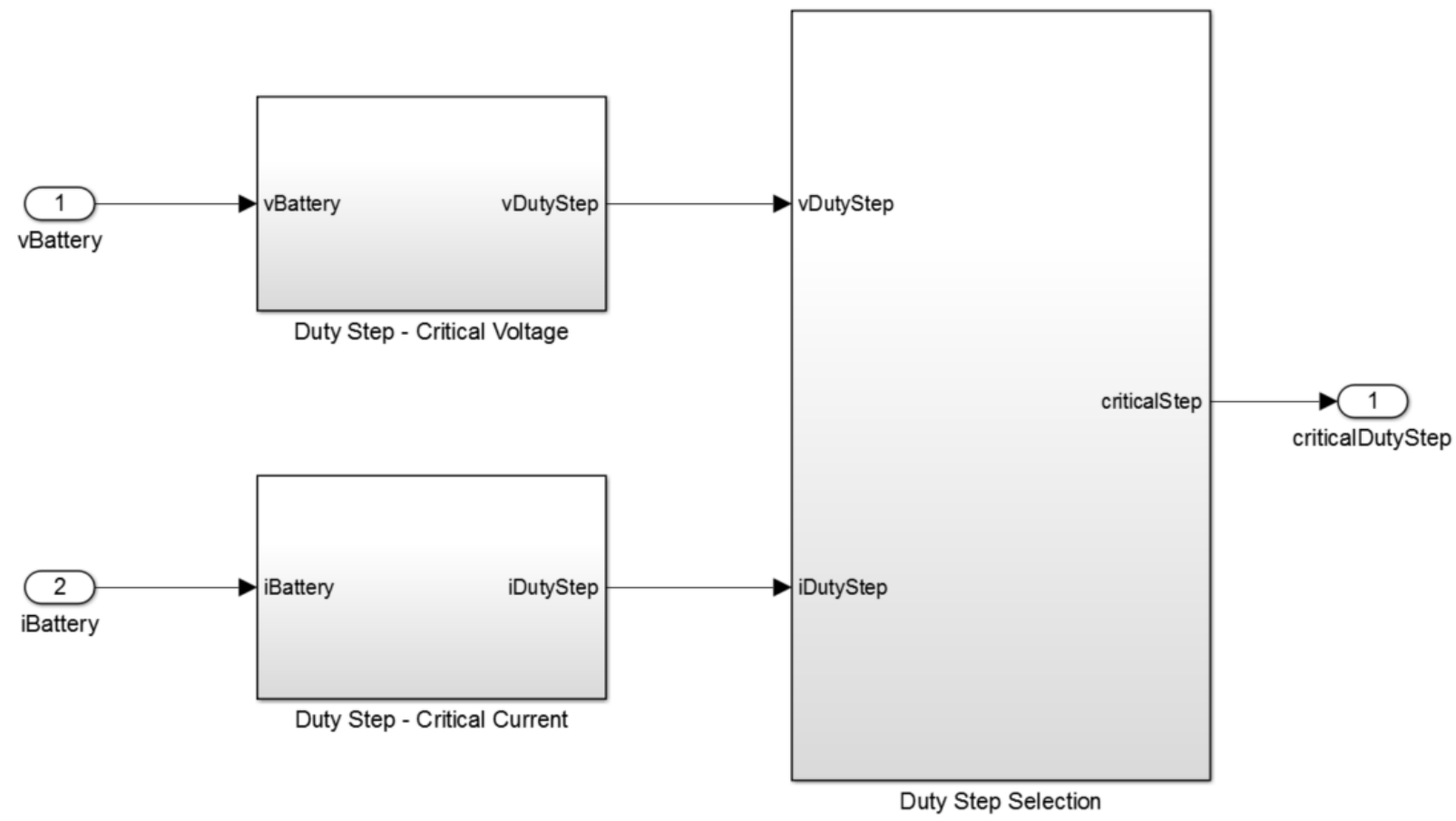

Figure 41. High Level Simulink Model of Duty Step - Critical

\section{A.2.2.1. Battery Voltage Control Block}

Duty Step - Critical Voltage block, Figure 42, calculates a duty step based on the scaled relationship between the battery's maximum voltage and actual voltage. As the battery voltage gets closer to the maximum voltage, the duty step size is reduced, and vice versa. To improve overall stability of the limit cases, a derivative element is included to further improve the convergence properties towards maximum voltage. This model represents equation 2. 


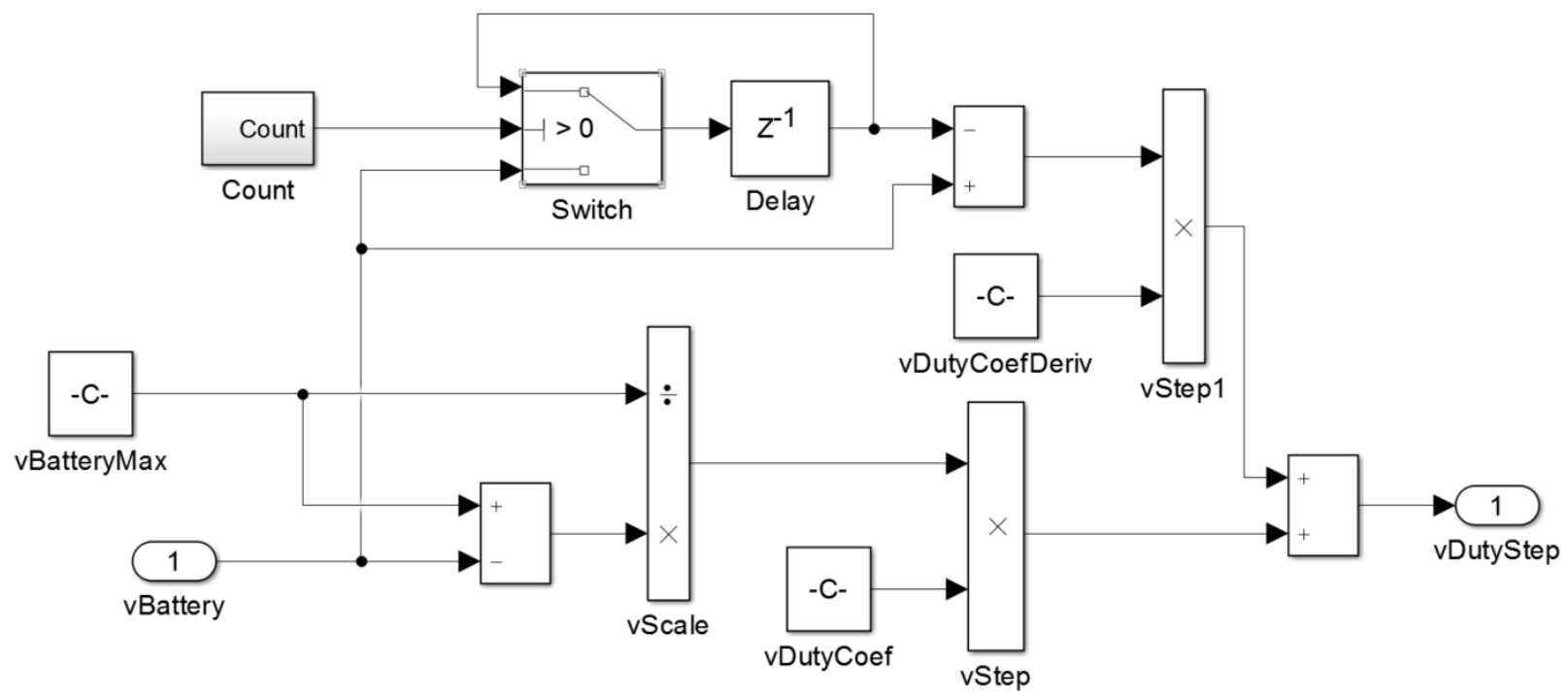

Figure 42. Simulink Model of Duty Step - Critical Voltage

\section{A.2.2.2. Battery Current Control Block}

Duty Step - Critical Current block, Figure 43, calculates the duty step similarly to the voltage case but with comparison between the battery's maximum current and actual current. Because this block is not necessarily required for the control system, it may be disabled during simulations to focus on MPPT and end state charging (with the battery voltage control block). A derivative element is included to further improve the convergence properties towards maximum current. This model represents equation 3. 


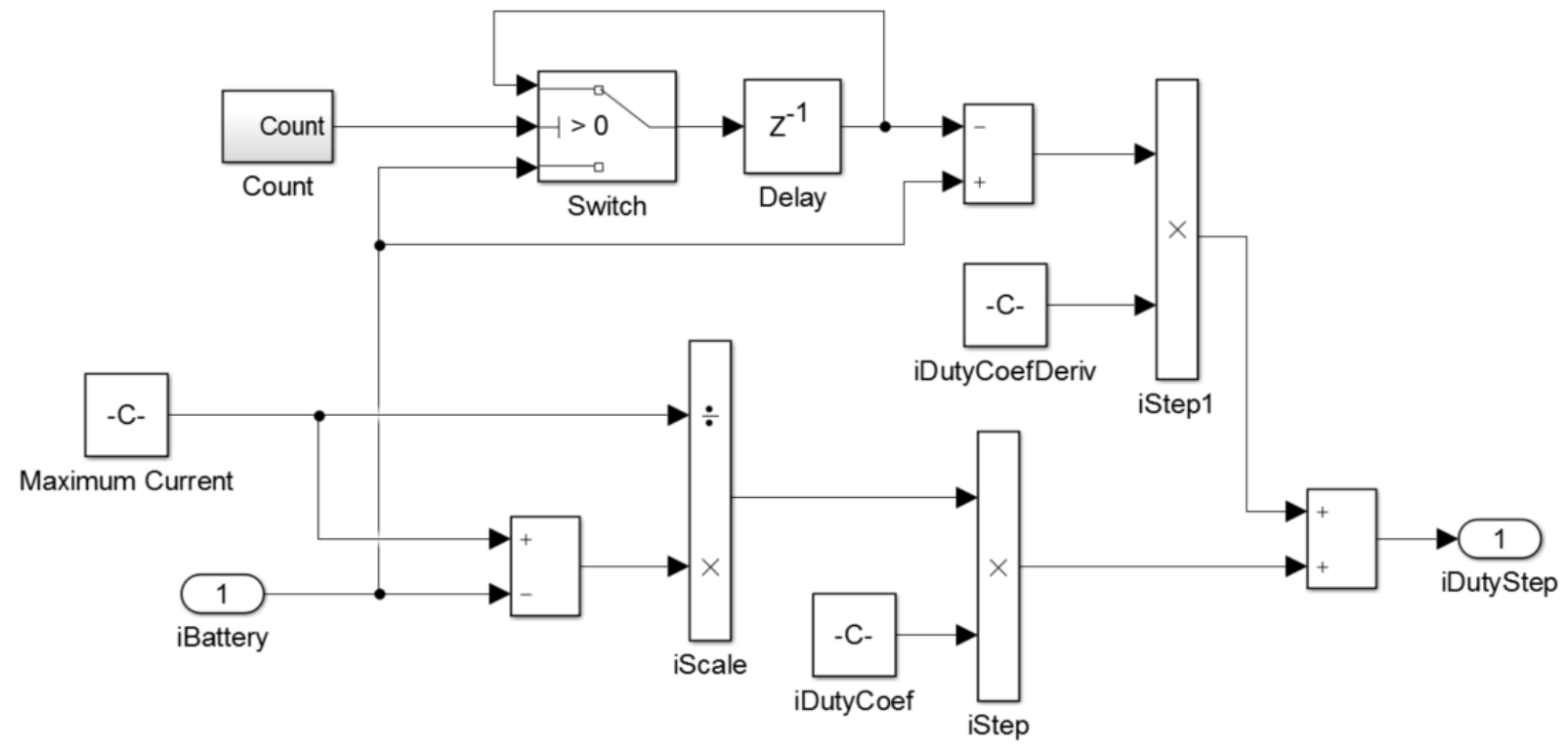

Figure 43. Simulink Model of Duty Step - Critical Current

\section{A.2.3. Duty Cycle Selection and Duty Cycle Separation Blocks}

The Critical Duty Step Selection block, Figure 44, selects the lowest value of the two critical duty steps; the lowest value is always the more critical case.

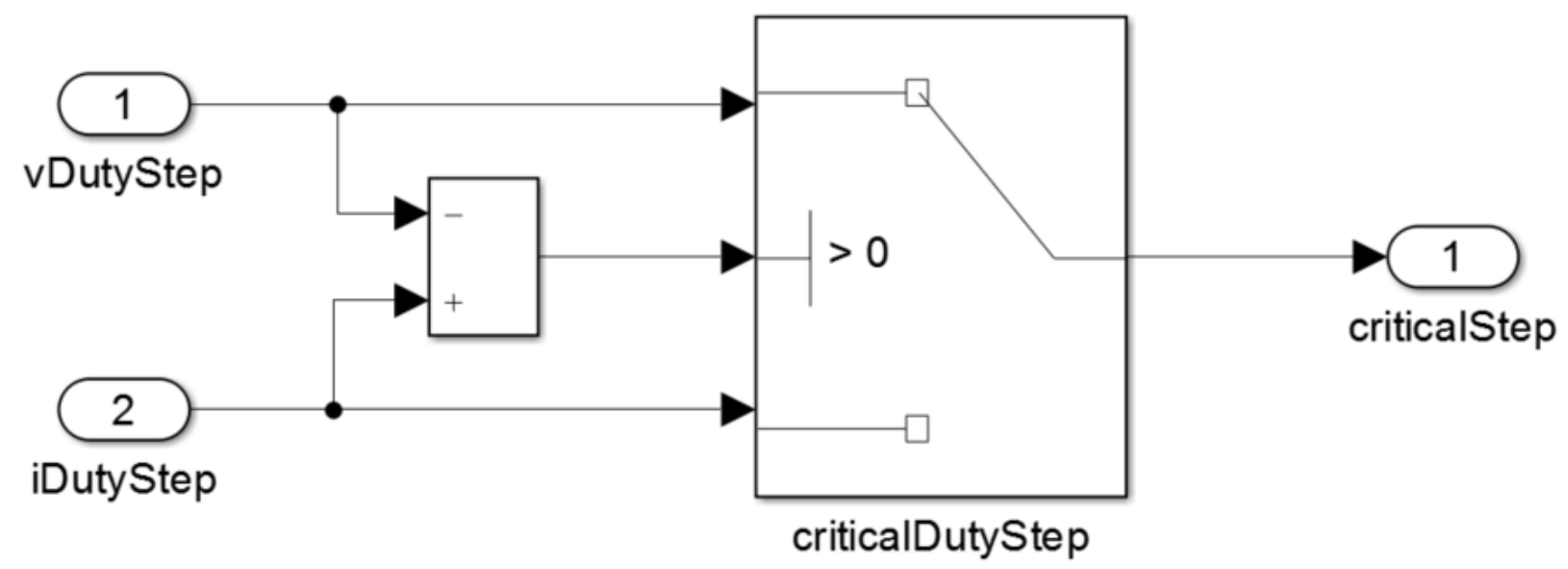

Figure 44. Simulink Model of Critical Duty Step Selection 
The Duty Cycle Selection block, Figure 45, selects the proper duty cycle to send to the power converter; the smallest duty cycle is the selected duty cycle as this is the most important.

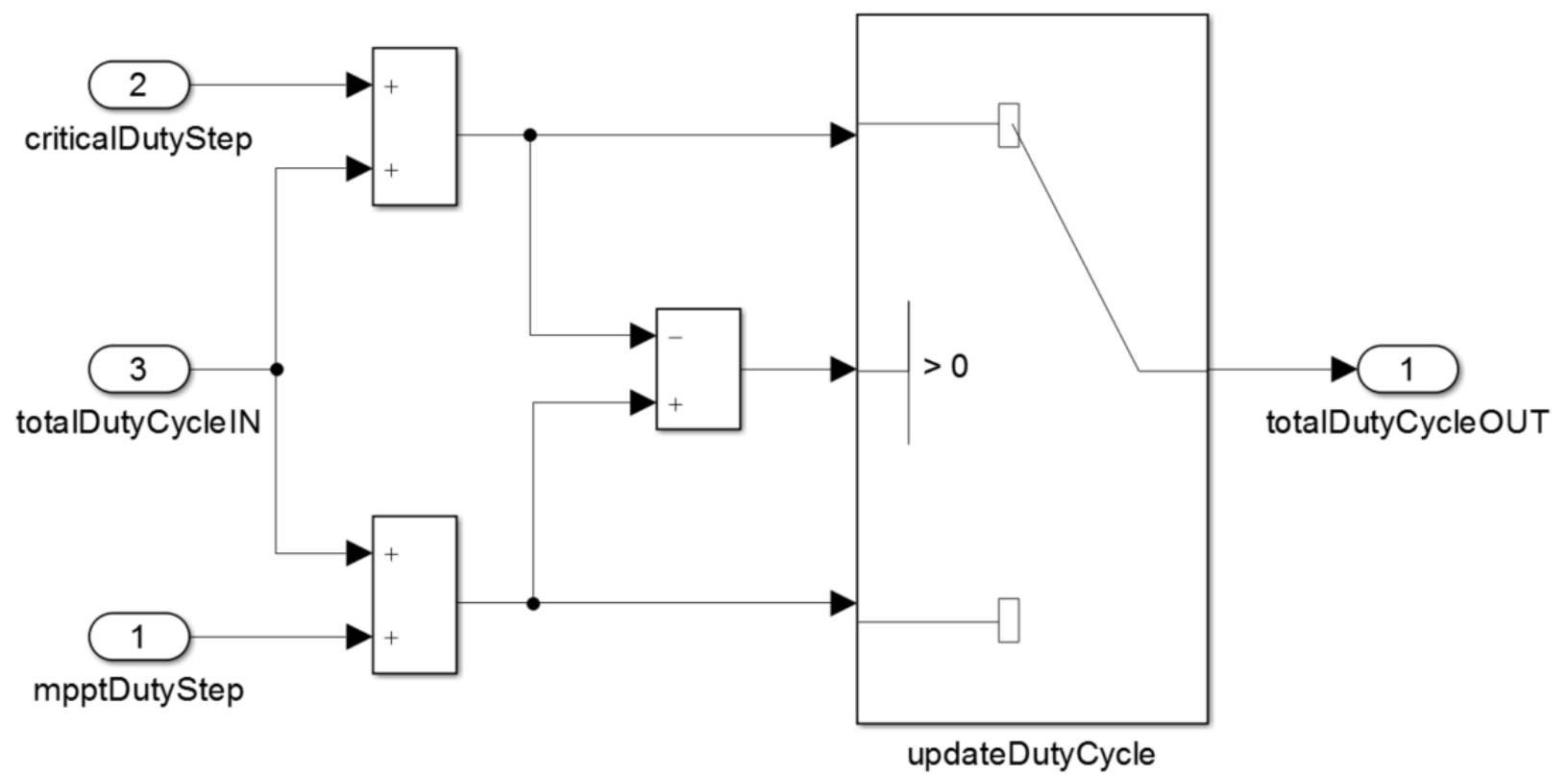

Figure 45. Simulink Model of Duty Cycle Selection

The Duty Cycle Routing block, Figure 46, uses a total duty cycle signal of two, which is Buck Duty Cycle plus Boost Duty Cycle. The Buck Duty Cycle is chosen as the value of the total duty cycle if the total is less than one. The Boost Duty Cycle is chosen as the magnitude of the total duty cycle over one. The Buck Duty Cycle is one when in boosting mode, and the Boost Duty Cycle is zero when in bucking mode. This set-up works nicely for this converter because to reduce the power, the duty cycle is stepped down, which can inherently put the system into the buck mode and vice versa. 


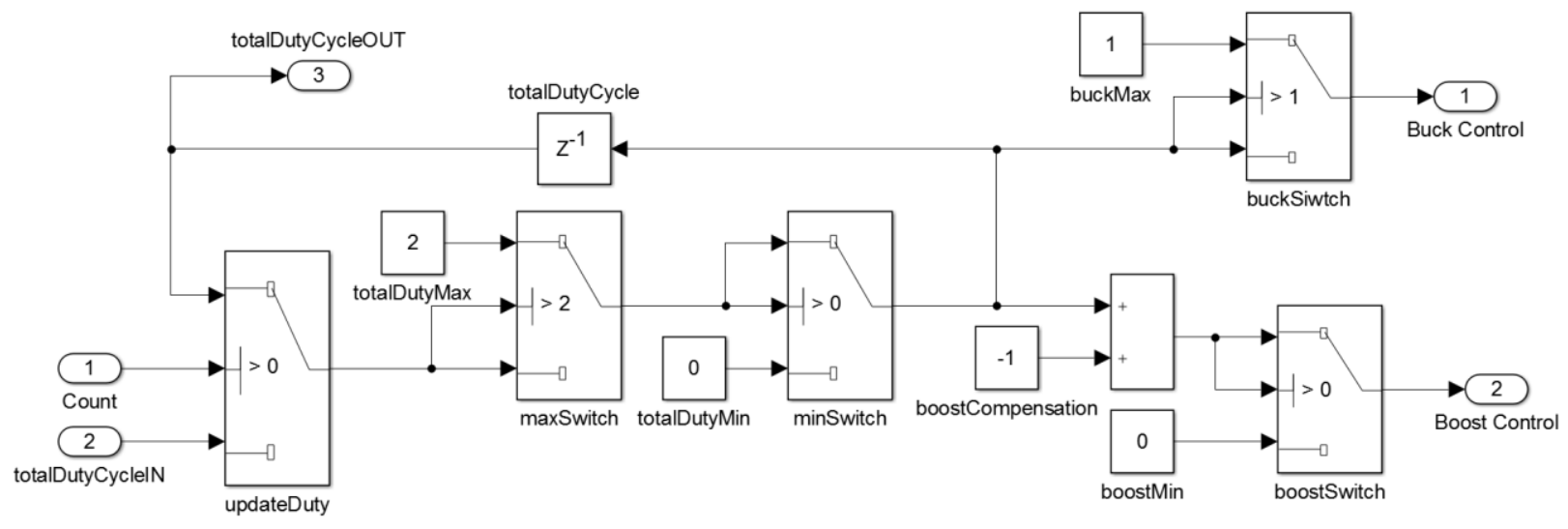

Figure 46. Simulink Model of Duty Cycle Routing 


\section{Appendix B. Parameter Test Cases}

This appendix contains the test cases of the equations presented in Chapter 4. These test cases are further investigated in order to select the parameters relative to the specific solar panel and power converter circuit.

\section{B.1. MPPT Control Test Cases}

Parameters $a$ and $b$ of equation 1 are investigated in this section.

$$
\Delta D_{M P P T}=a \frac{d P}{d V}+b
$$

\section{B.1.1. Parameter $a$ Test Cases}

For all test cases in this section, parameter $b$ is set to zero.

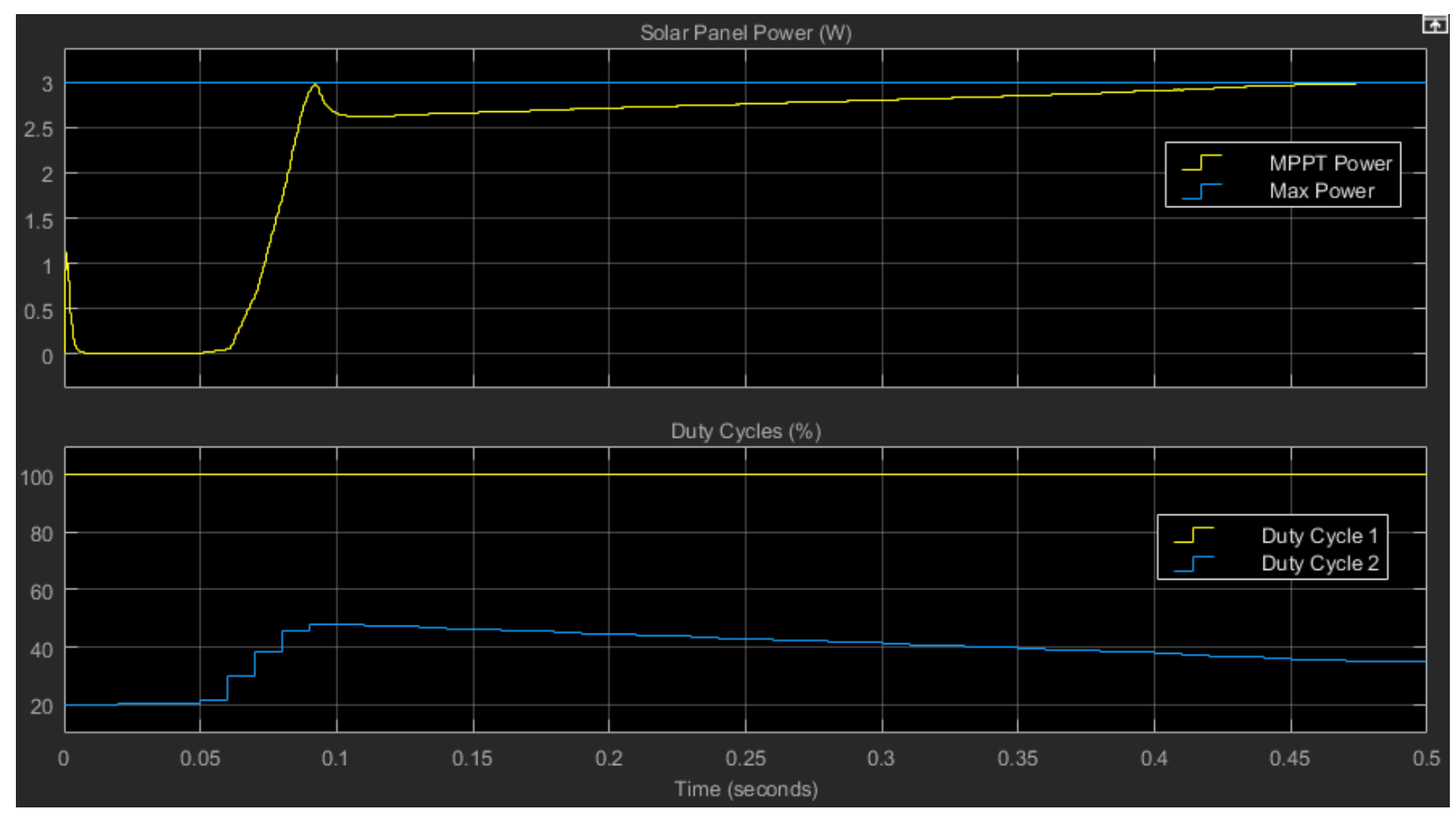

Figure 47. Case: $a=-0.005, b=0$ 

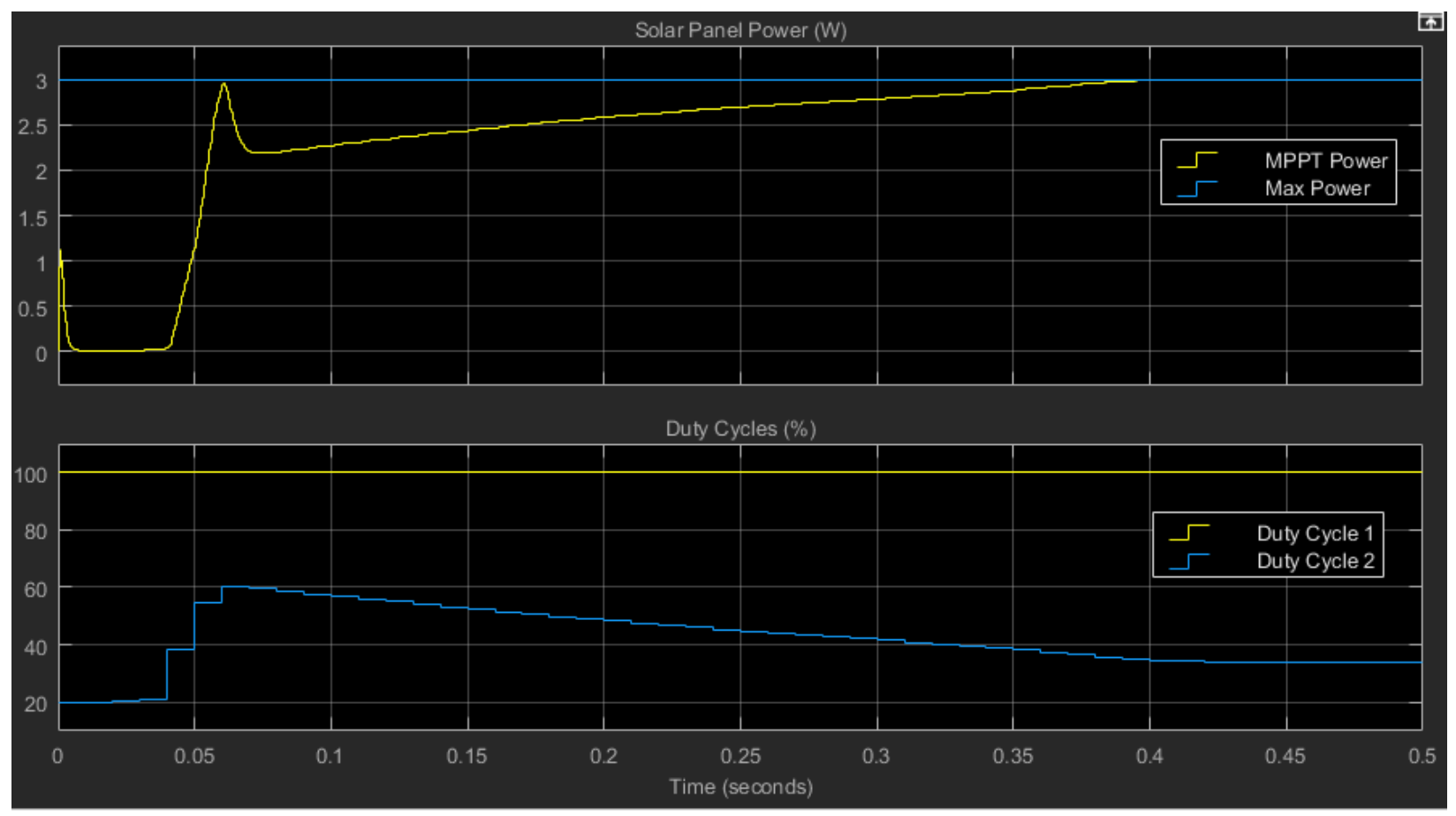

Figure 48. Case: $a=-0.01, b=0$
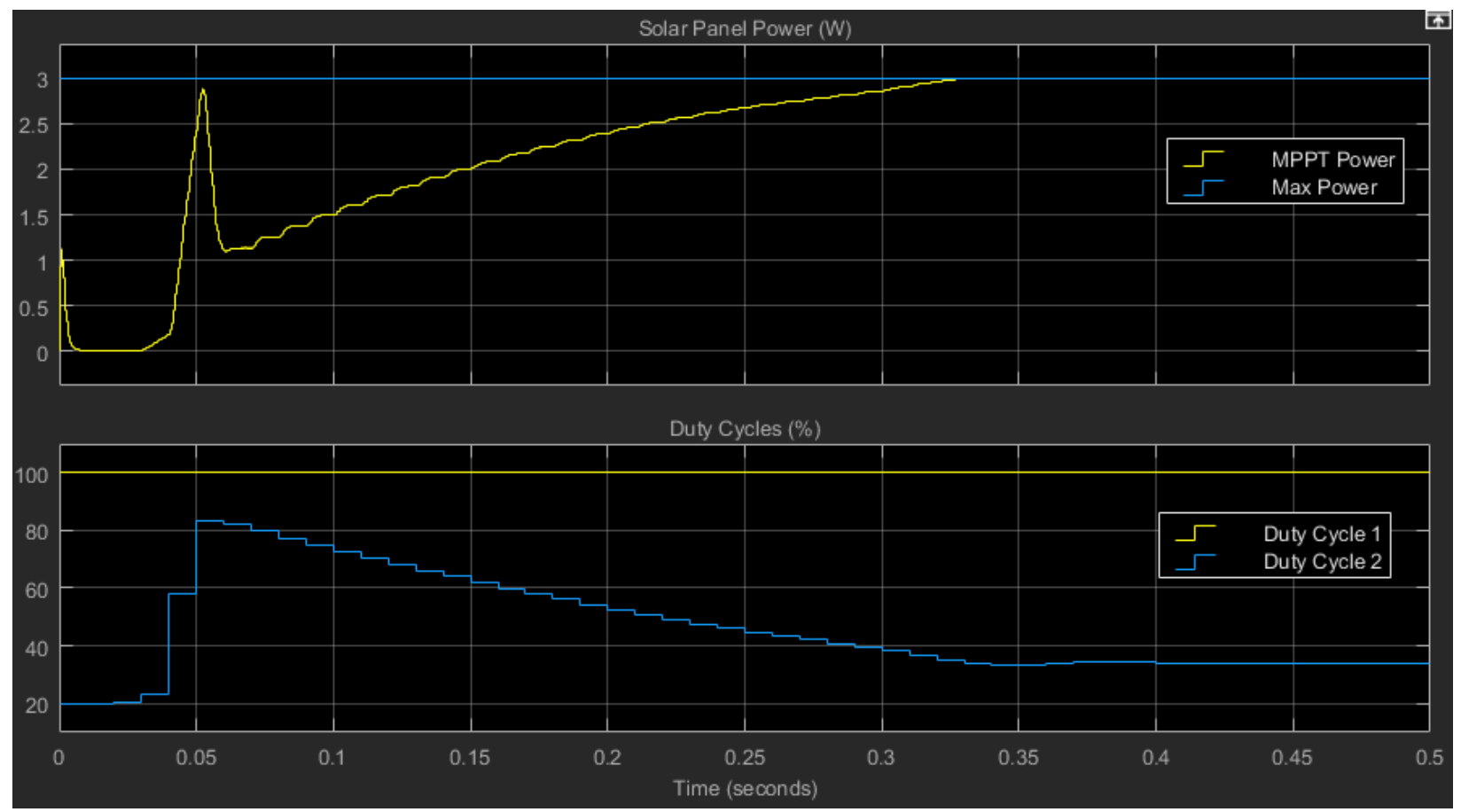

Figure 49. Case: $a=-0.02, b=0$ 

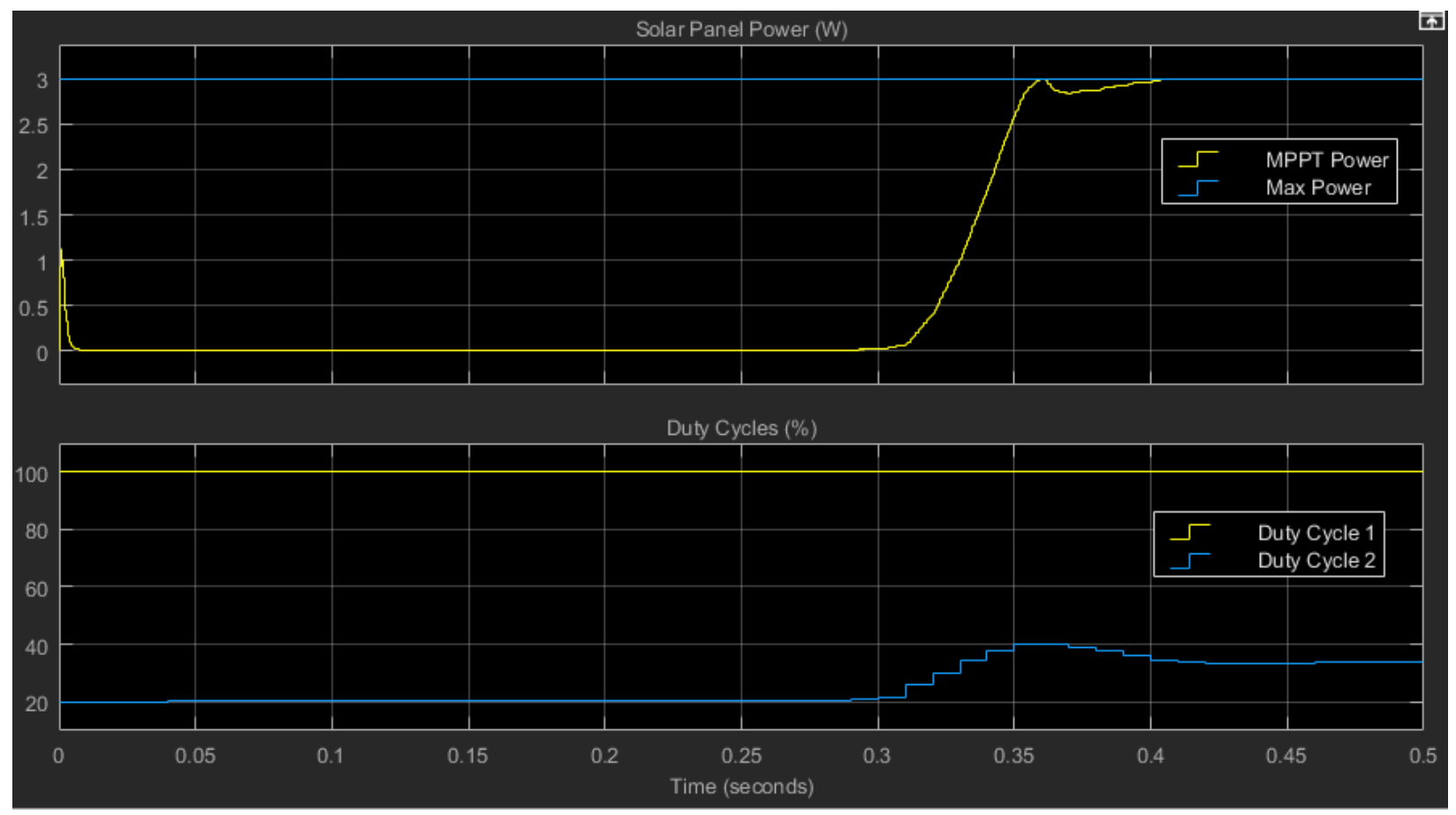

Figure 50. Case: $a+=-0.0025, a-=-0.02, b=0$
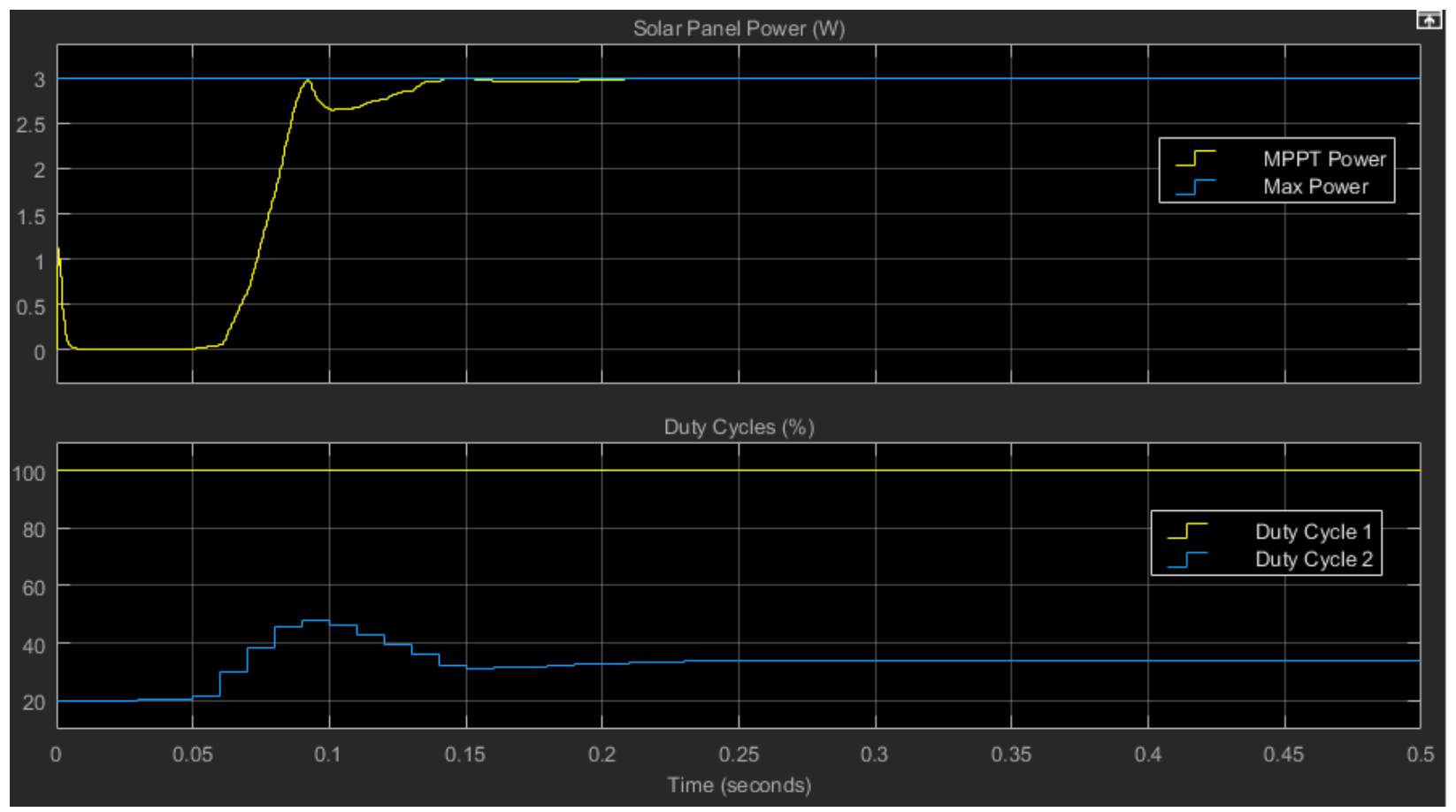

Figure 51. Case: $a+=-0.005, a-=-0.05, b=0$ 
B.1.2. Parameter $b$ Test Cases

For all test cases in this section, parameter $a$ is set to zero.

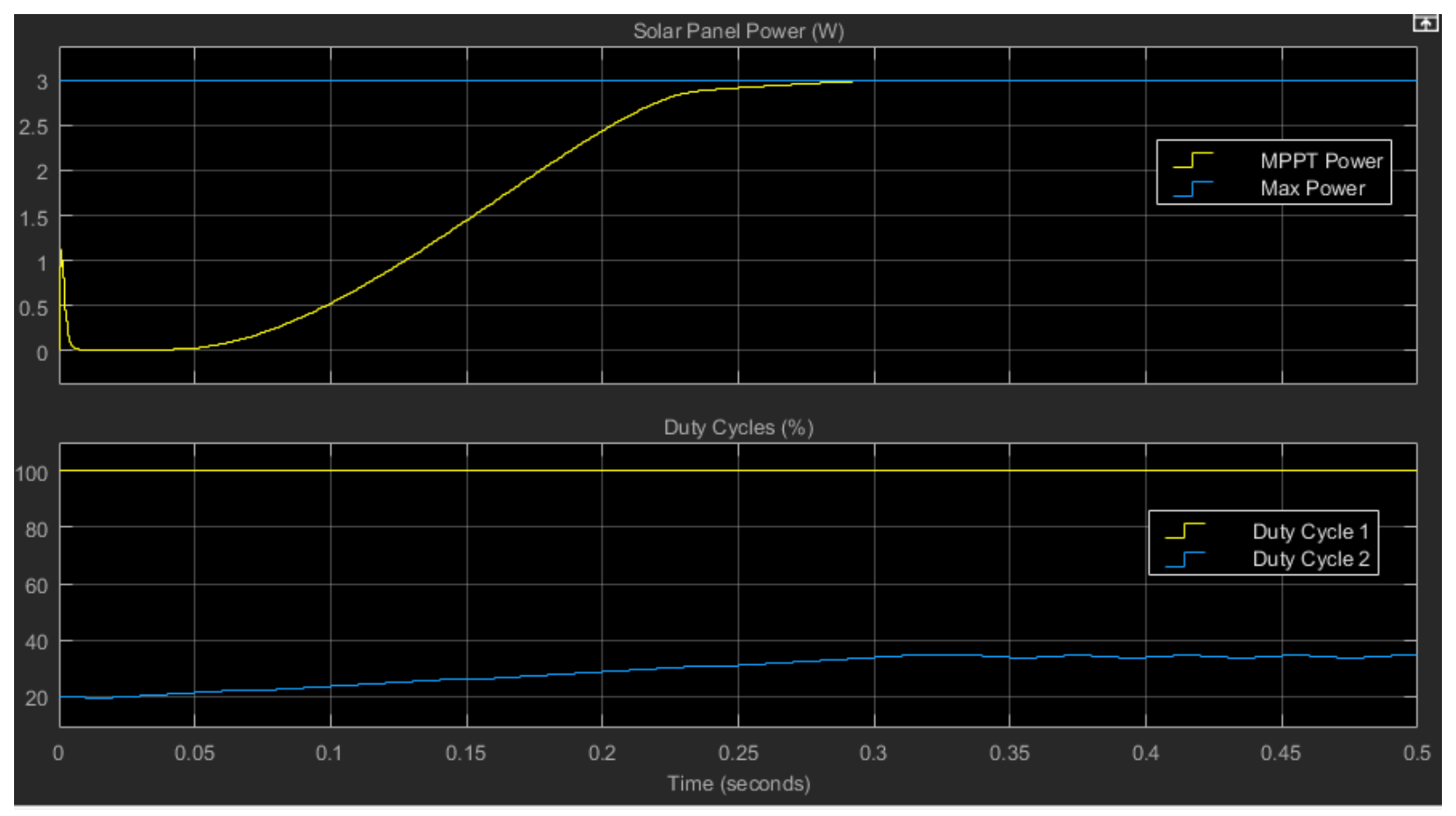

Figure 52. Case: $a=0,|b|=0.005$ 


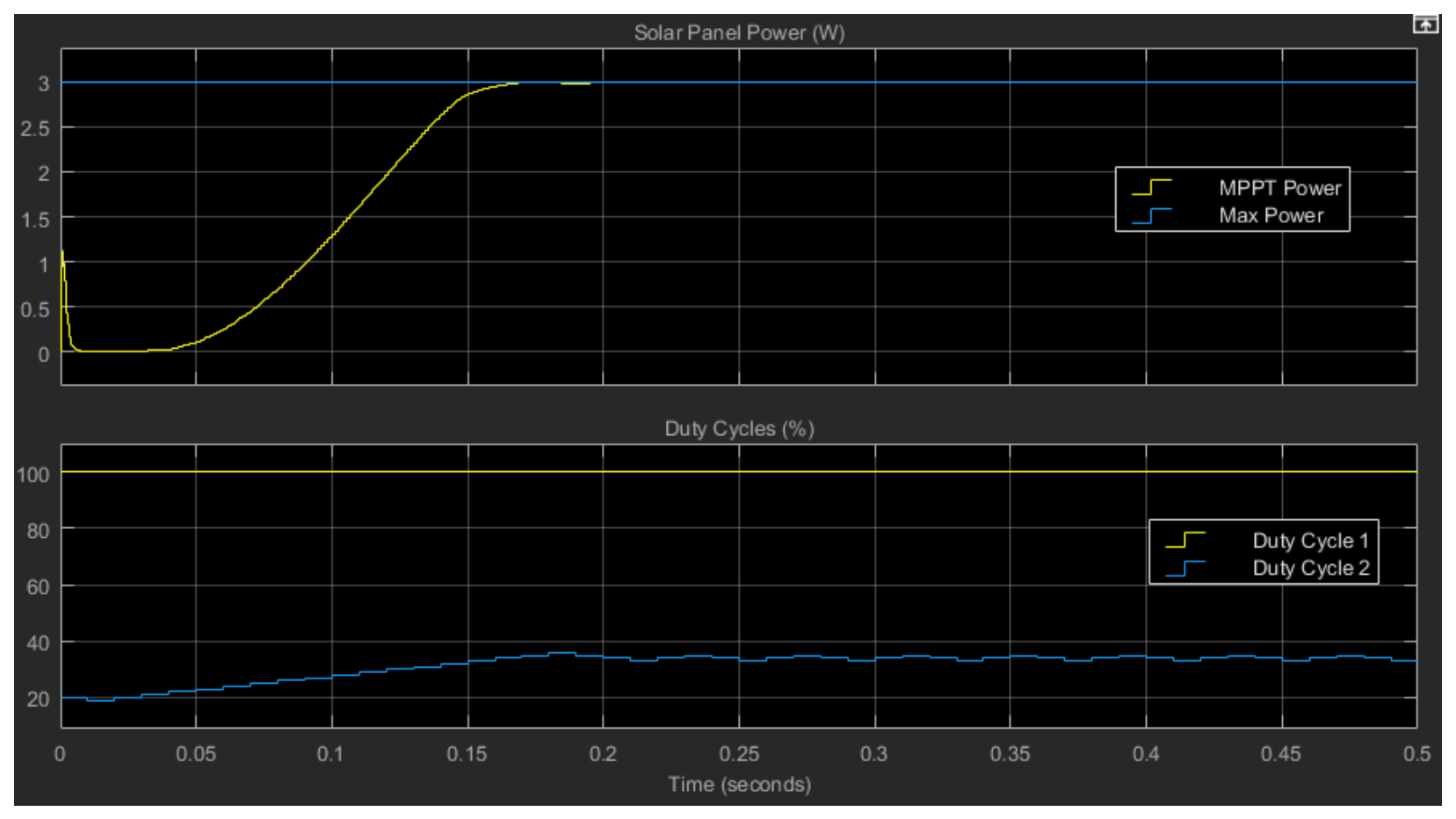

Figure 53. Case: $a=0,|b|=0.01$

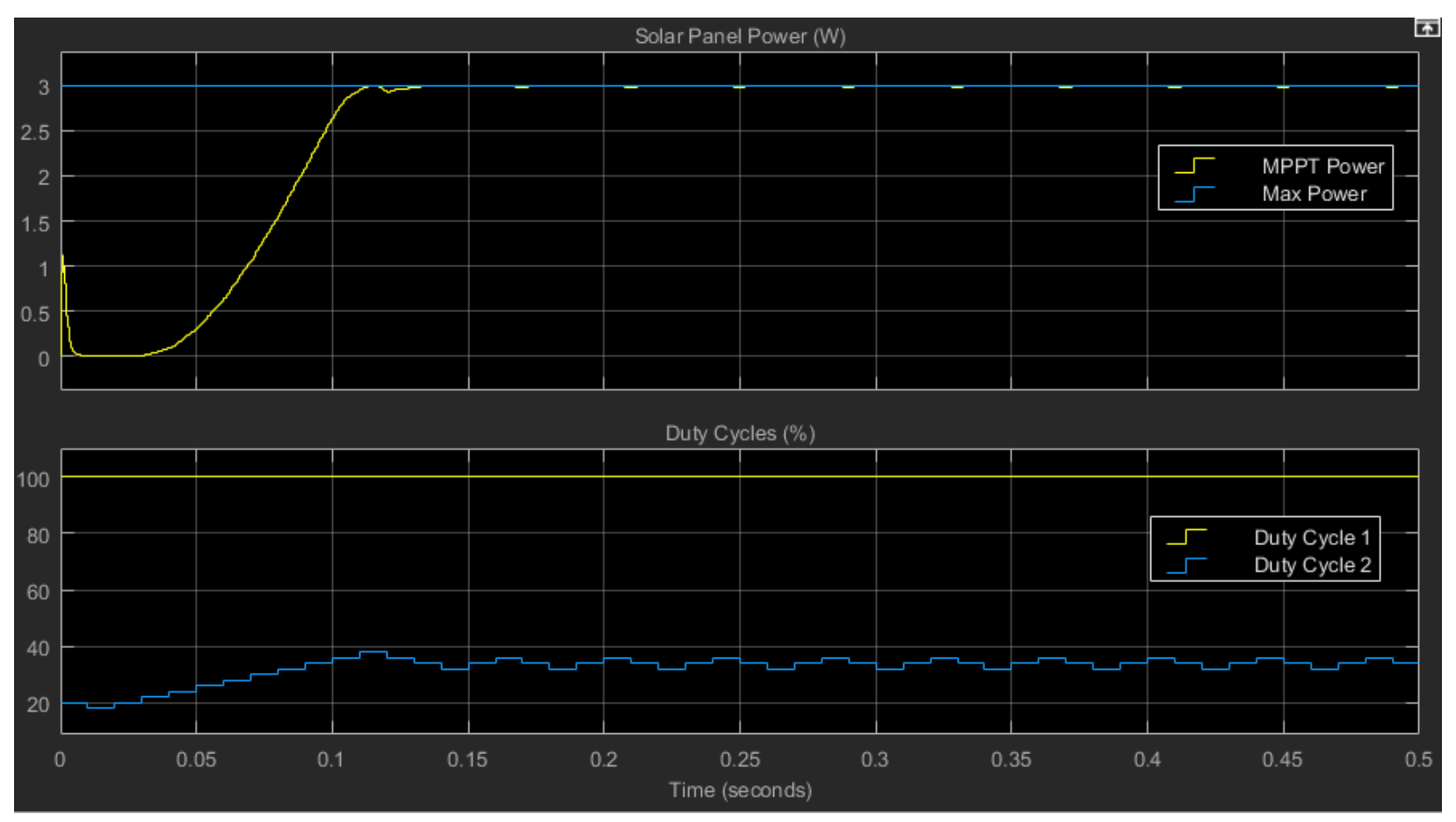

Figure 54. Case: $a=0,|b|=0.02$ 


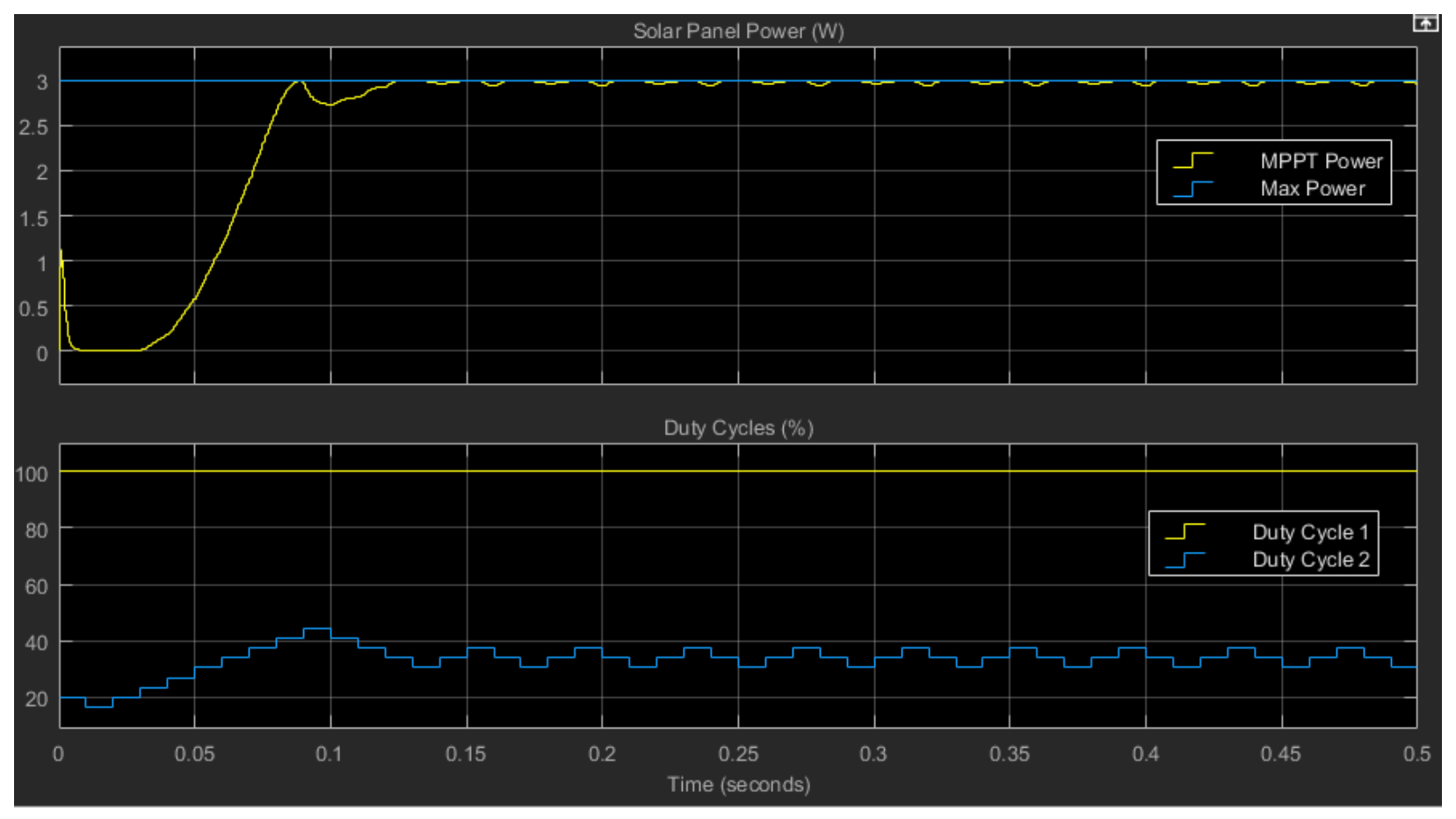

Figure 55. Case: $a=0,|b|=0.035$

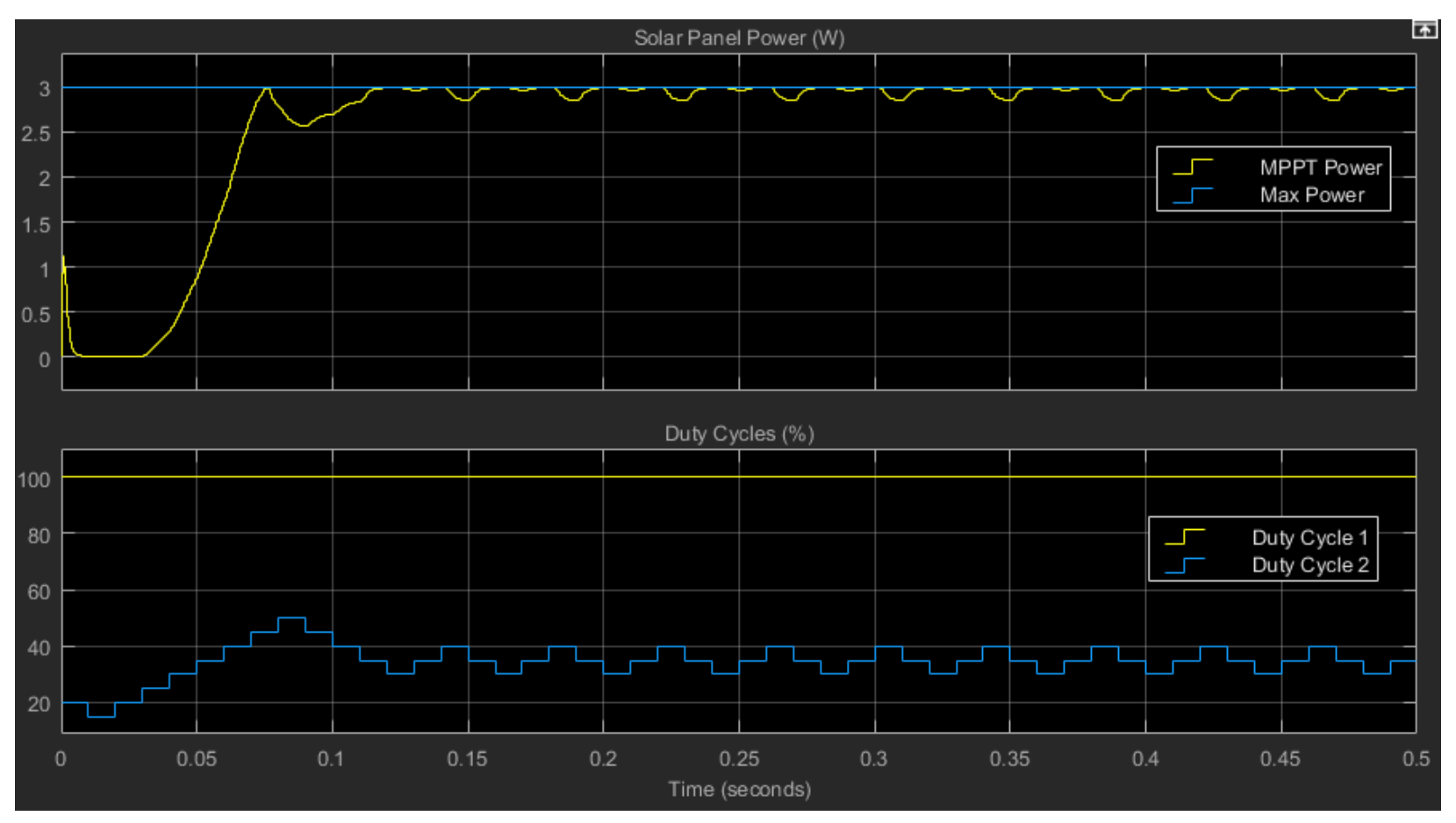

Figure 56. Case: $a=0,|b|=0.05$ 


\section{B.1.3. Parameter $a$ and $b$ Test Cases}

For all test cases in this section, parameter $a$ and $b$ aren't zero.

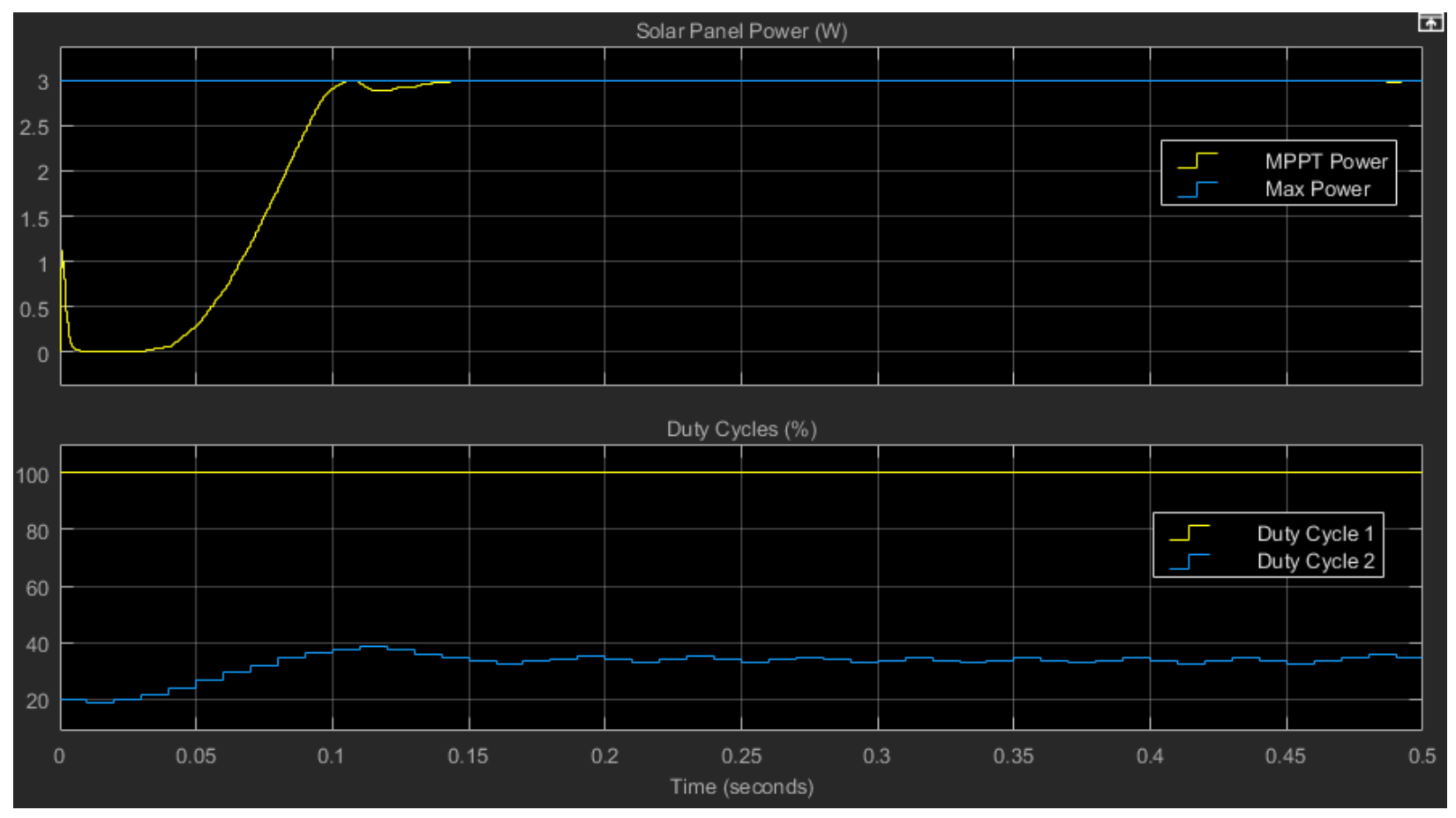

Figure 57. Case: $a+=-0.001, a-=-0.005,|b|=0.01$ 


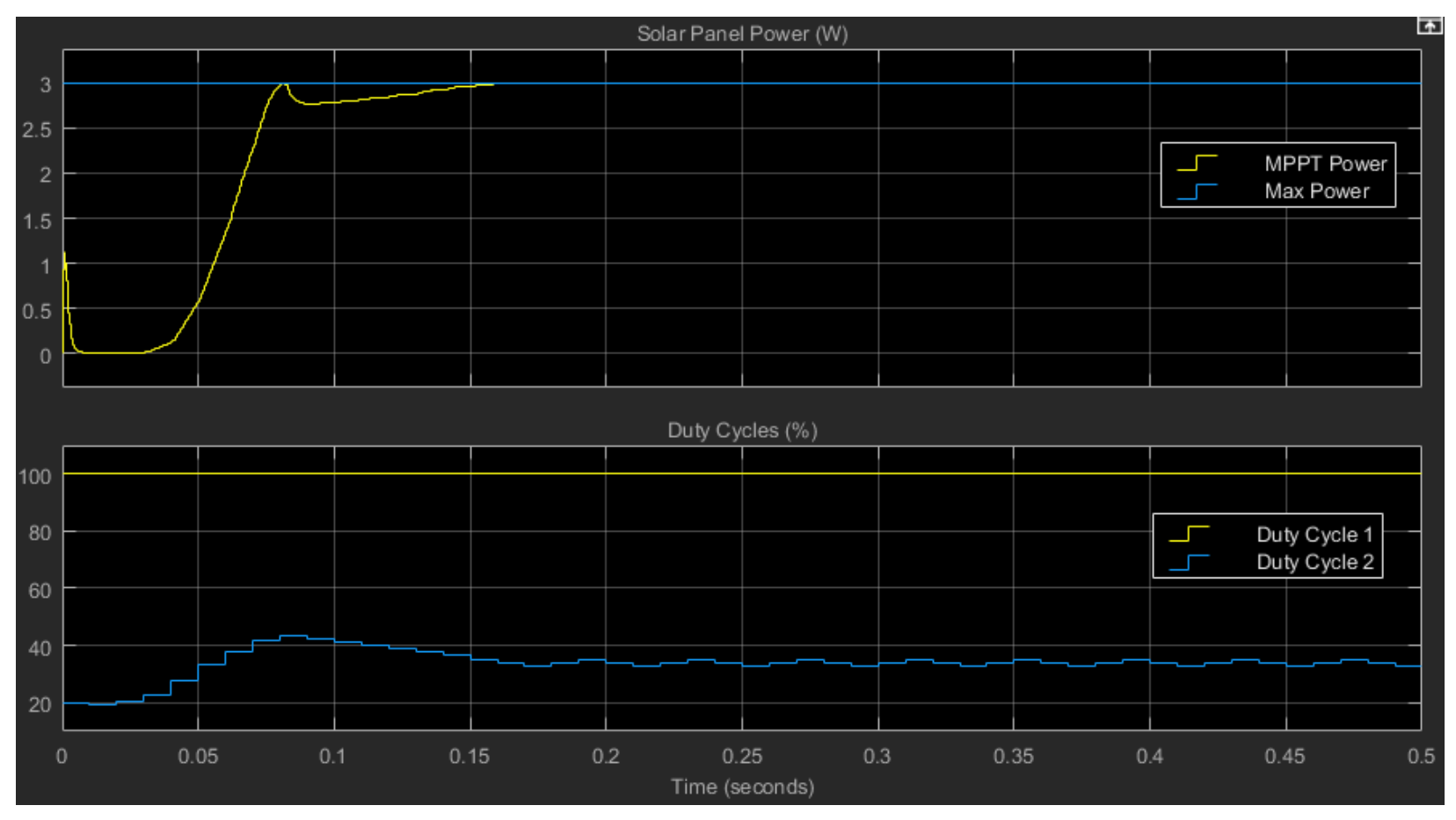

Figure 58. Case: $a=-0.0025,|b|=0.01$

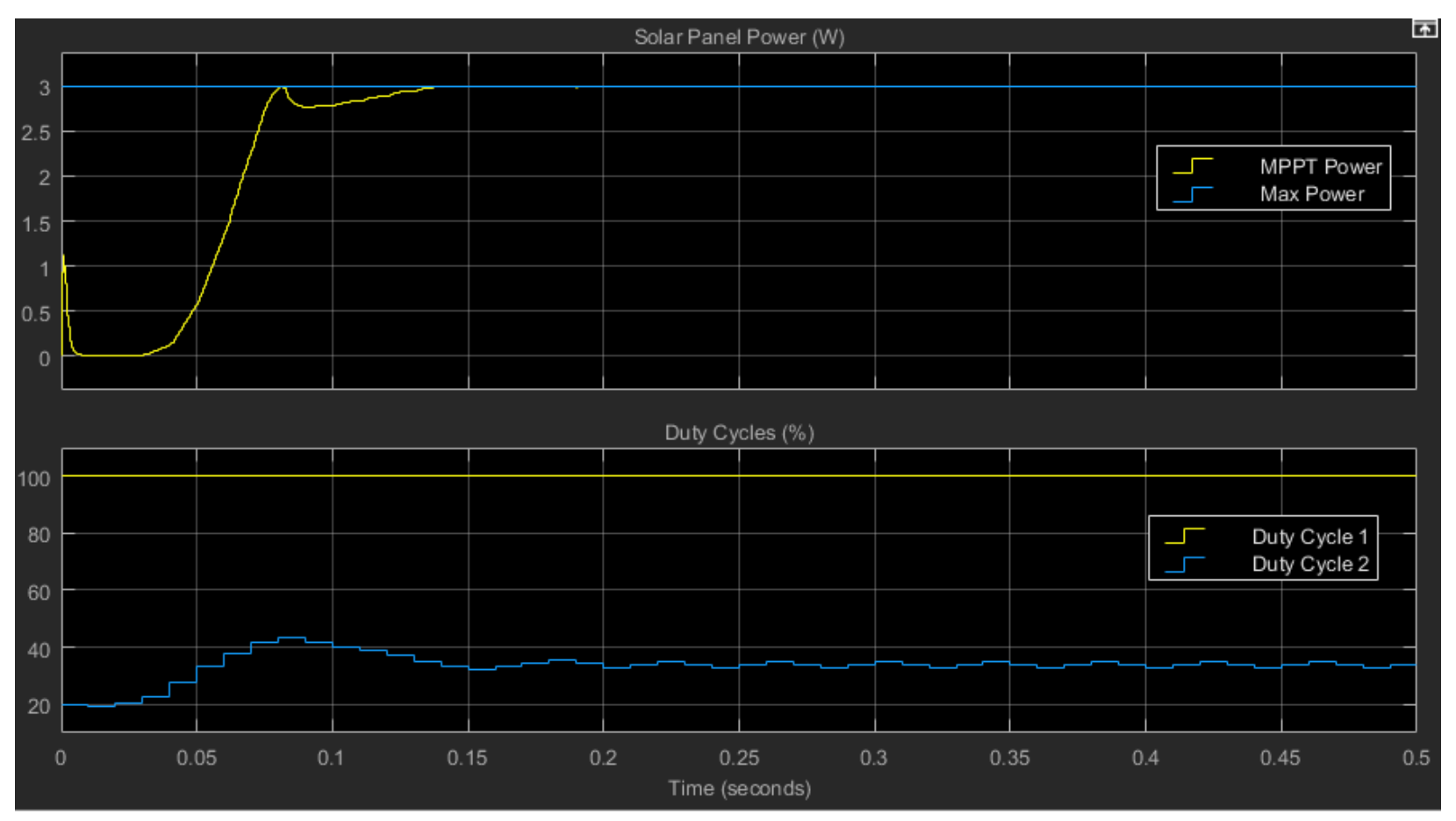

Figure 59. Case: $a+=-0.0025, a-=-0.01,|b|=0.01$ 


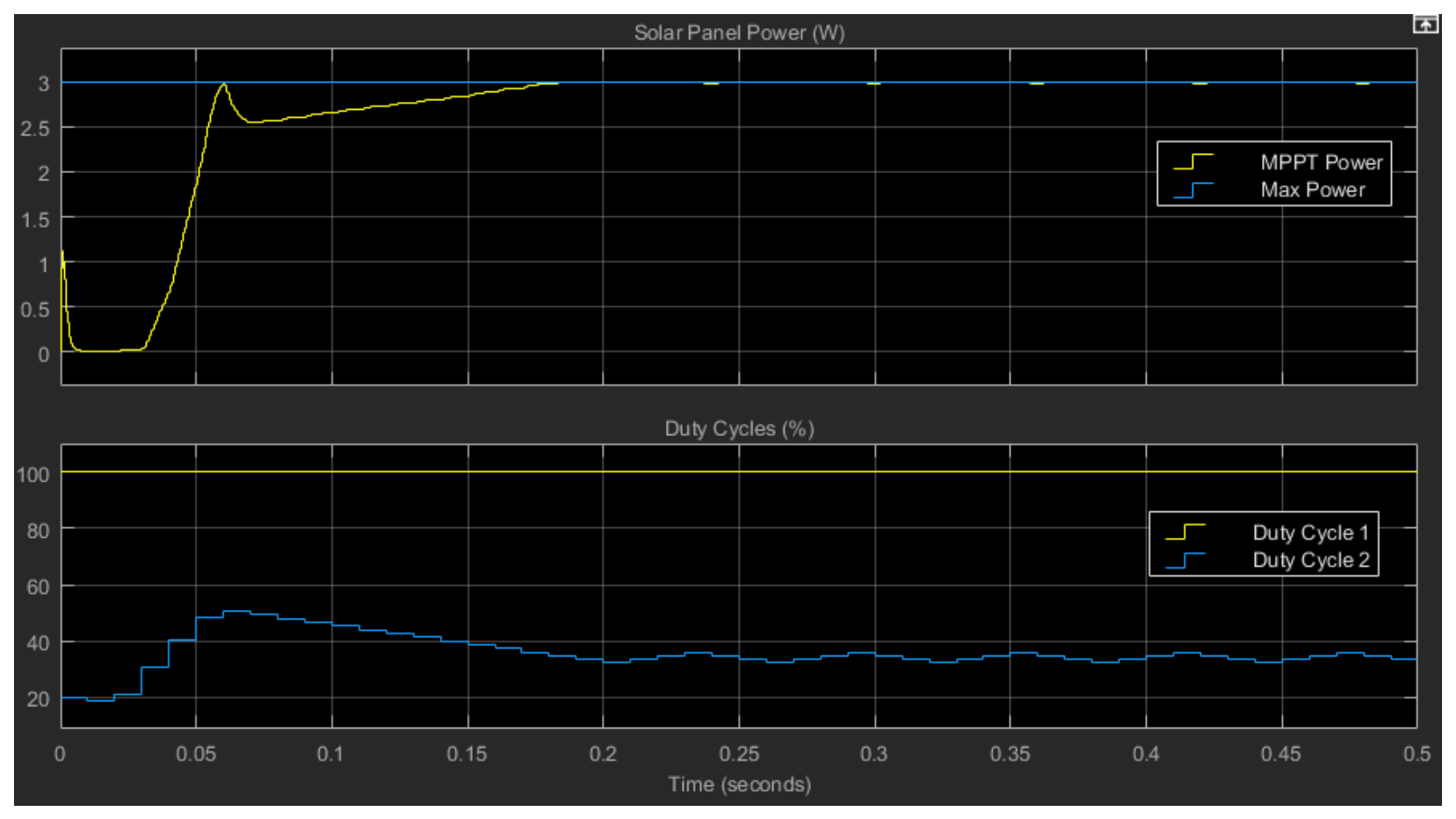

Figure 60. Case: $a=-0.005,|b|=0.01$

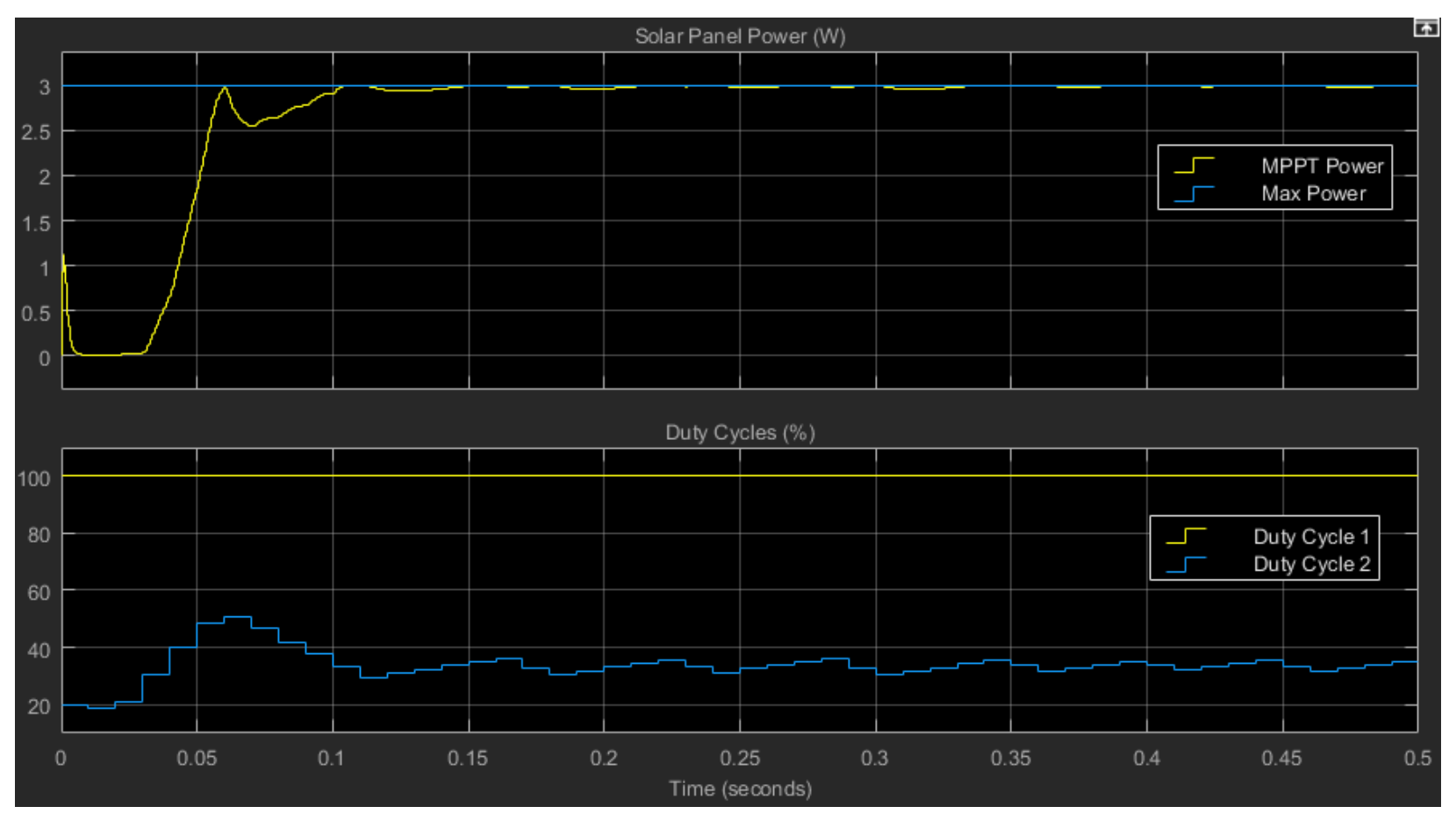

Figure 61. Case: $a+=-0.005, a-=-0.05,|b|=0.01$ 


\section{B.2. Battery Voltage Control Test Cases}

Parameters $c$ and $d$ of equation 2 are investigated in this section.

$$
\Delta D_{V}=c \frac{\left(V_{\max }-V_{B}\right)}{V_{\max }}+d\left(\Delta V_{B}\right)
$$

\section{B.2.1. Parameter $c$ Test Cases}

For all test cases in this section, parameter $d$ is set to zero.

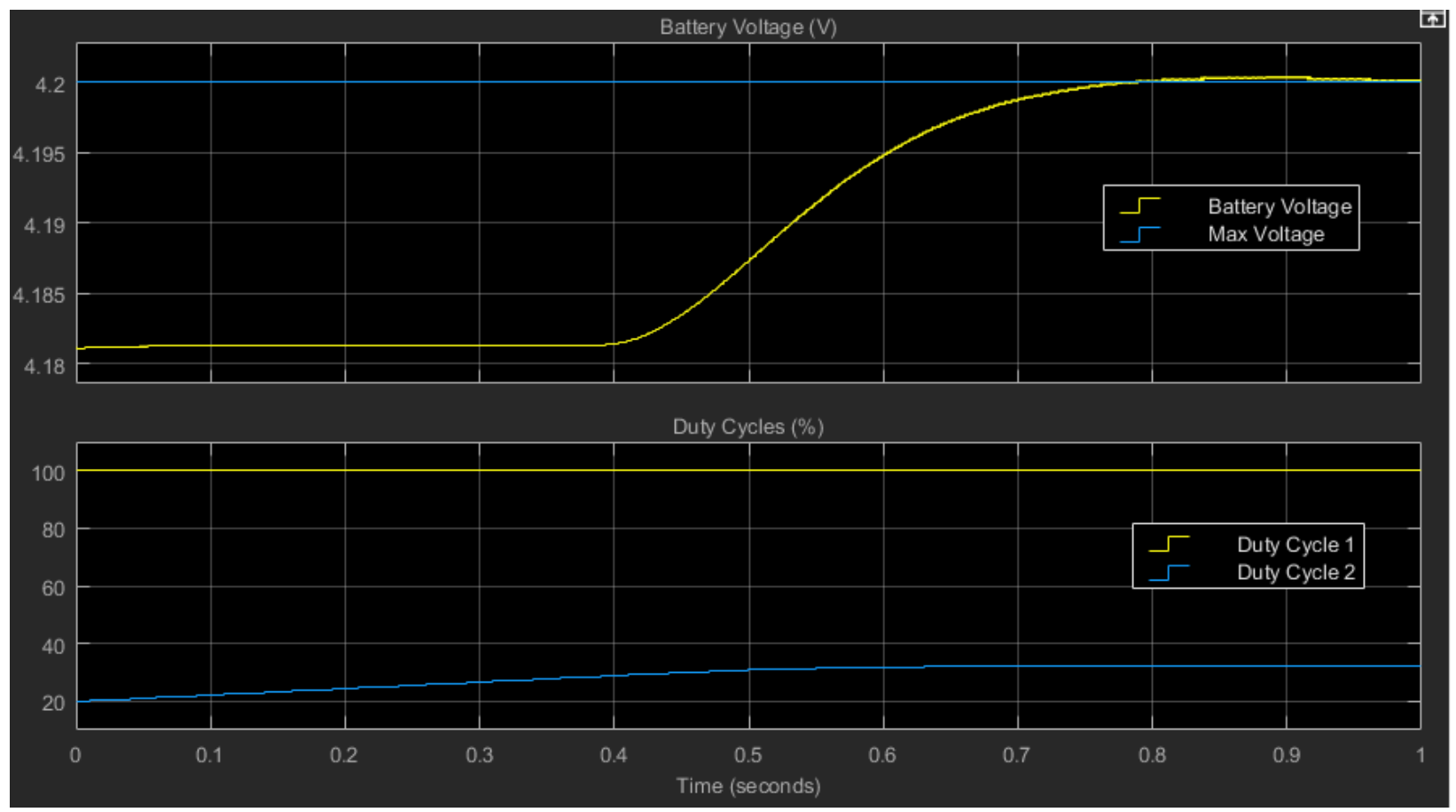

Figure 62. Case: $c=0.5, d=0$ 


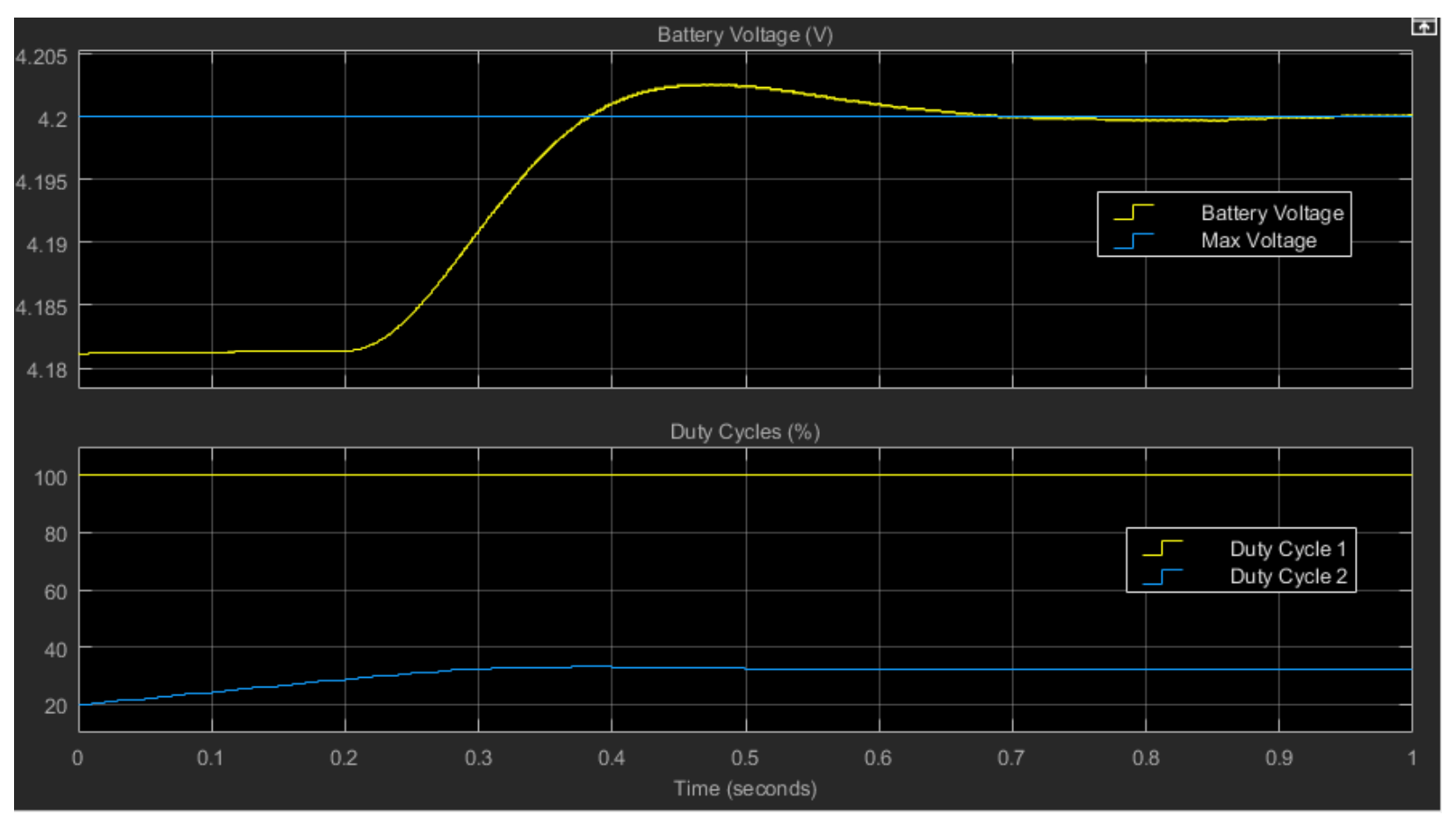

Figure 63. Case: $c=1, d=0$

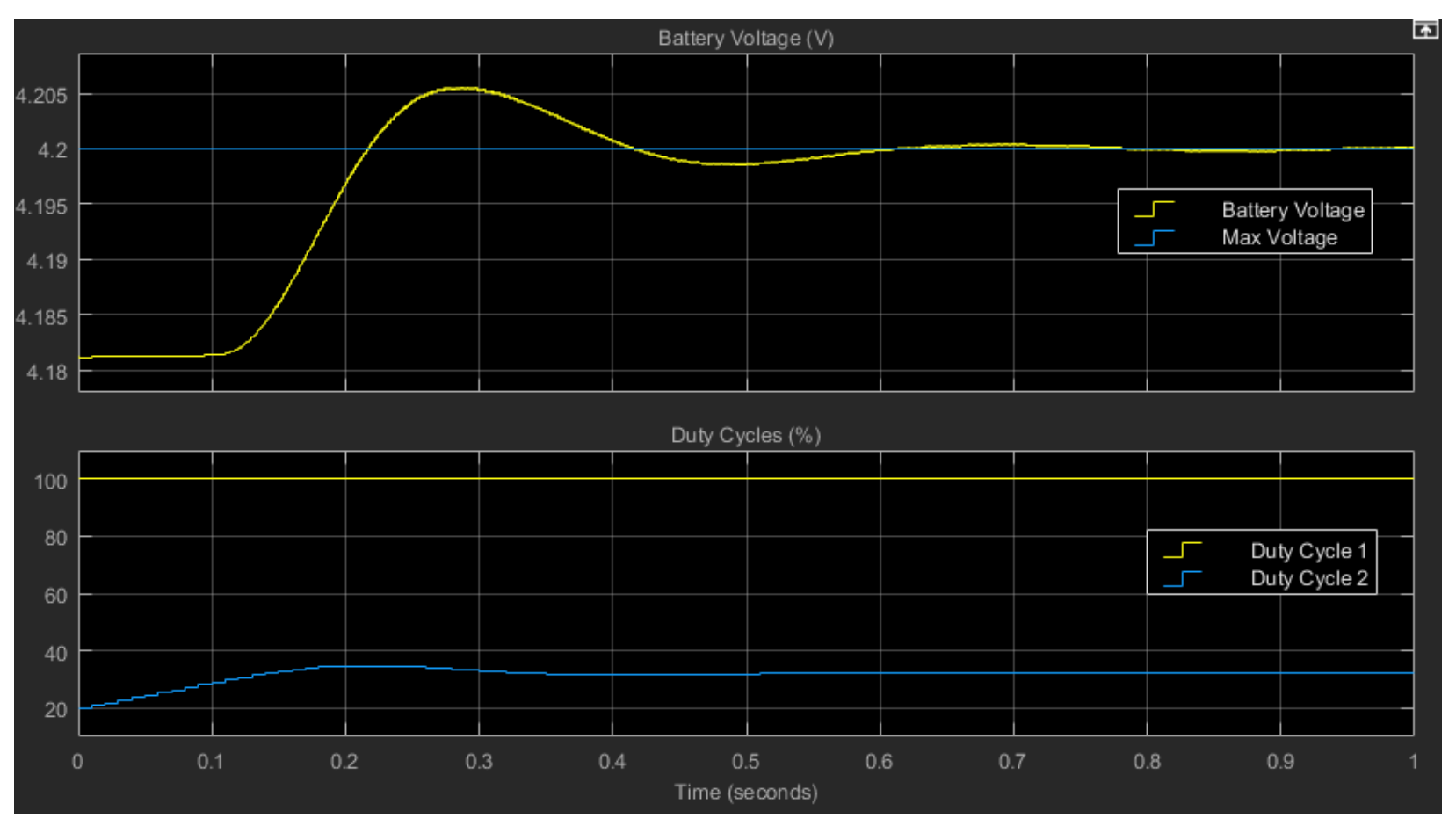

Figure 64. Case: $c=2, d=0$ 


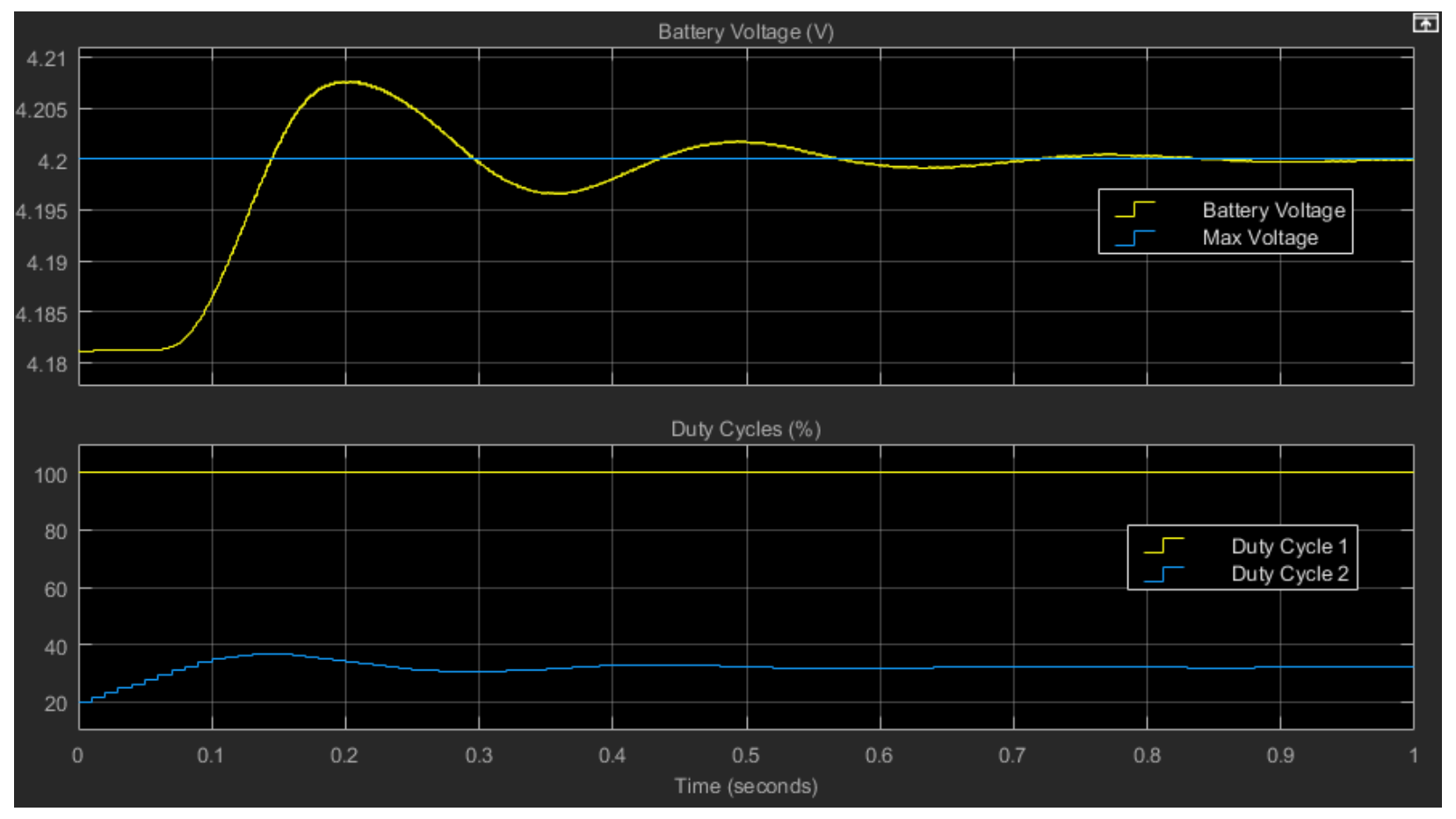

Figure 65. Case: $c=3.5, d=0$

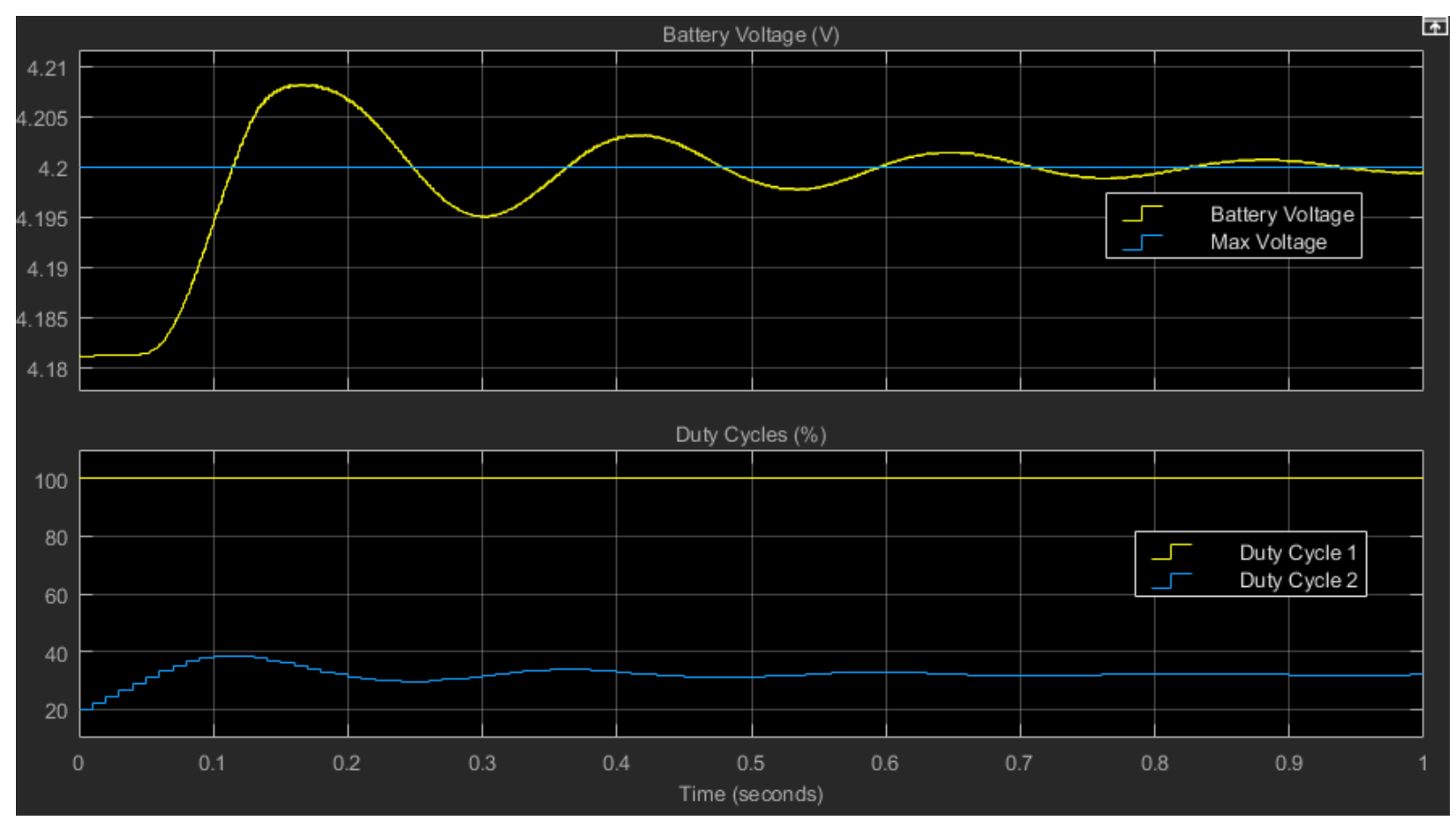

Figure 66. Case: $c=5, d=0$ 


\section{B.2.2. Parameter $c$ and $d$ Test Cases}

For all test cases in this section, parameter $c$ and $d$ aren't zero.

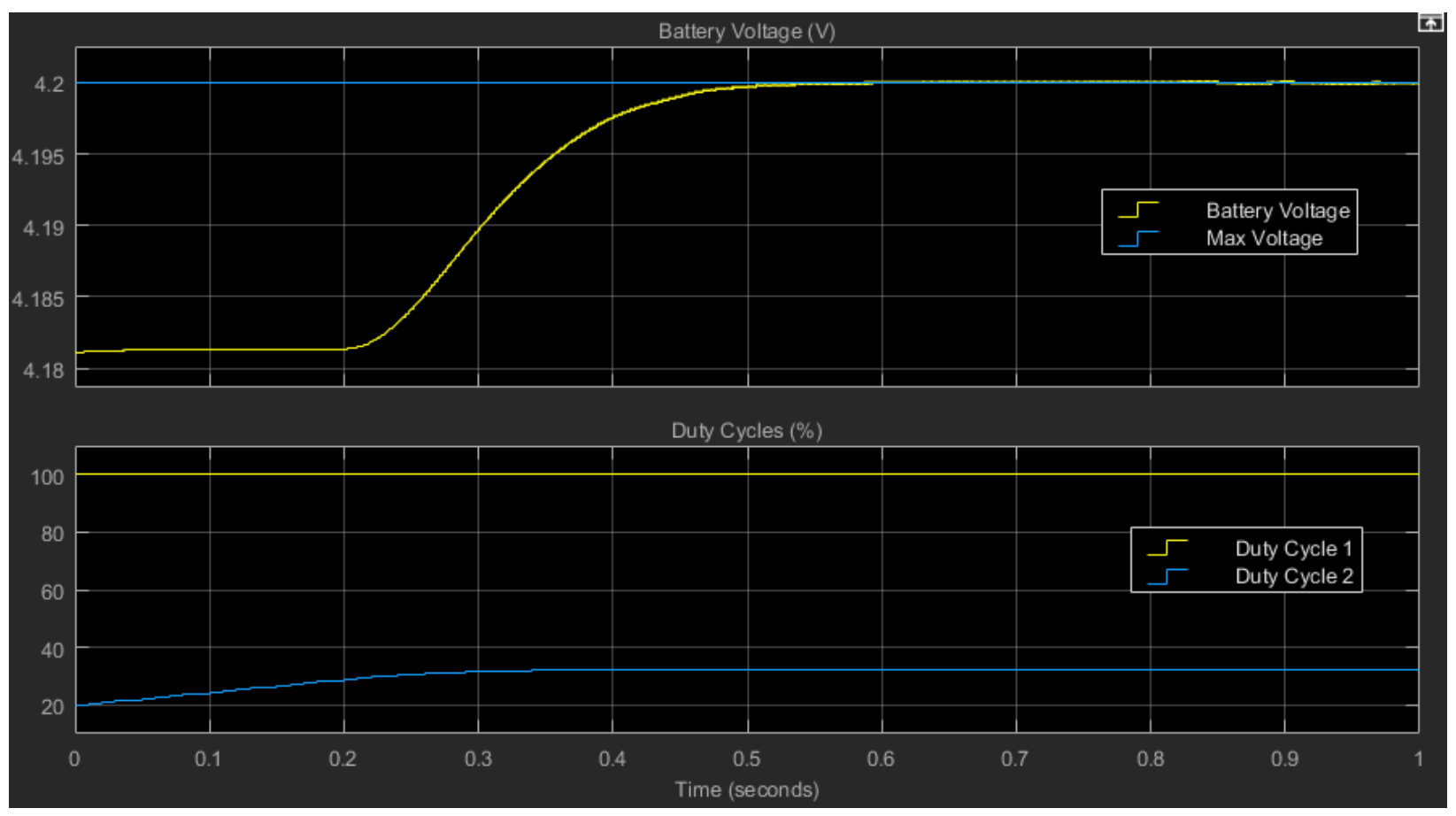

Figure 67. Case: $c=1, d=-1$ 


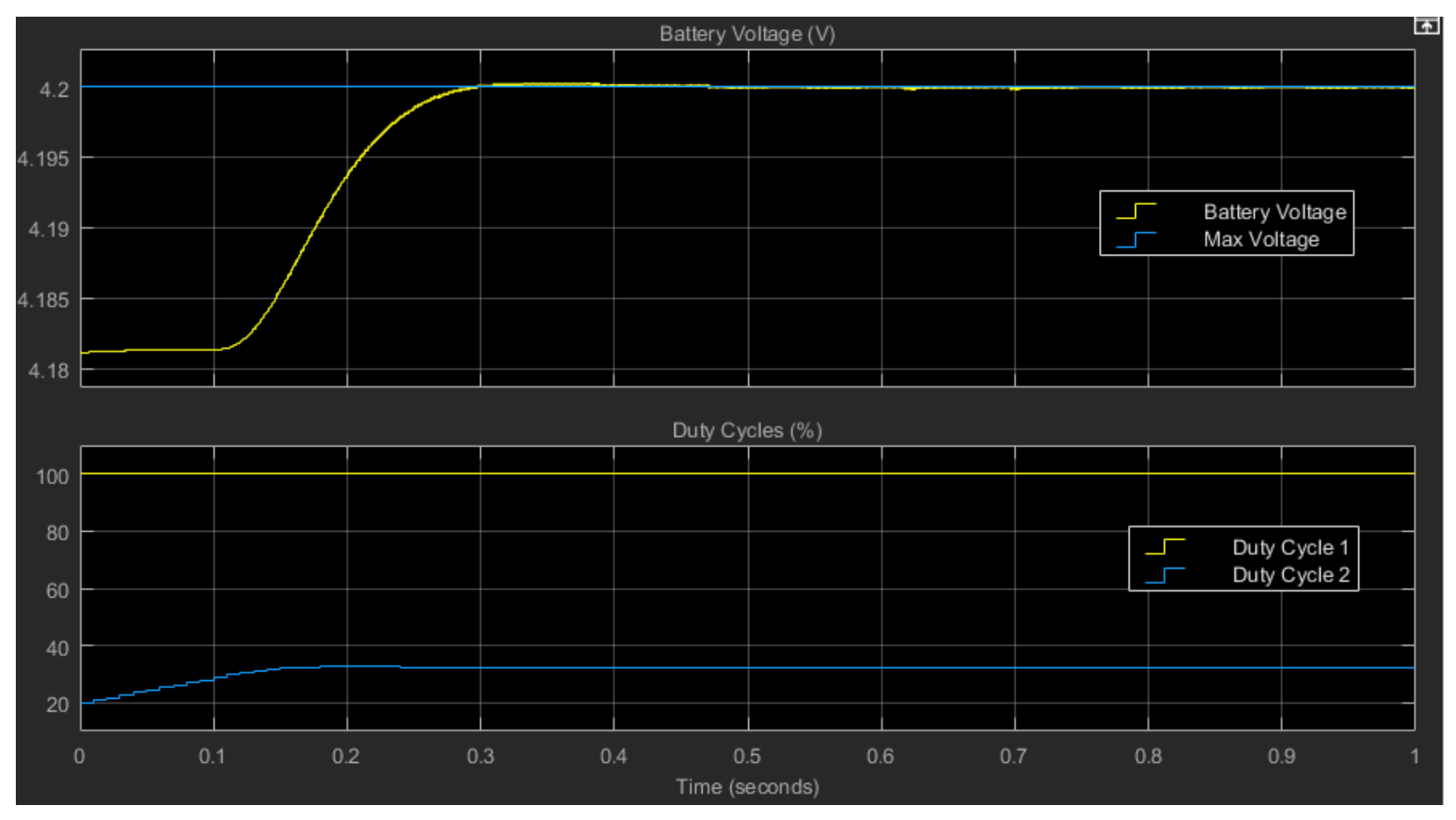

Figure 68. Case: $c=2, d=-2$

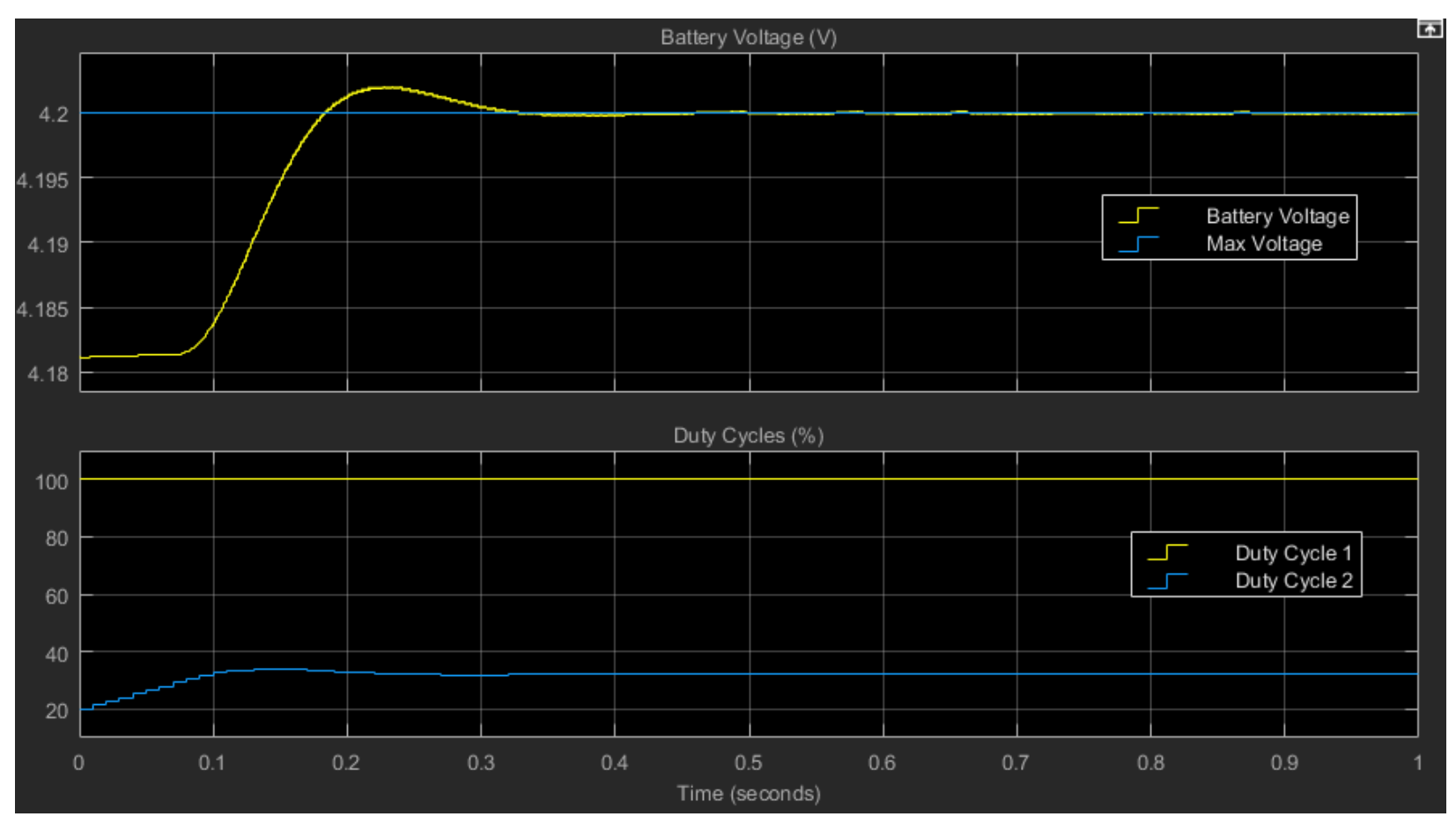

Figure 69. Case: $c=3, d=-2$ 


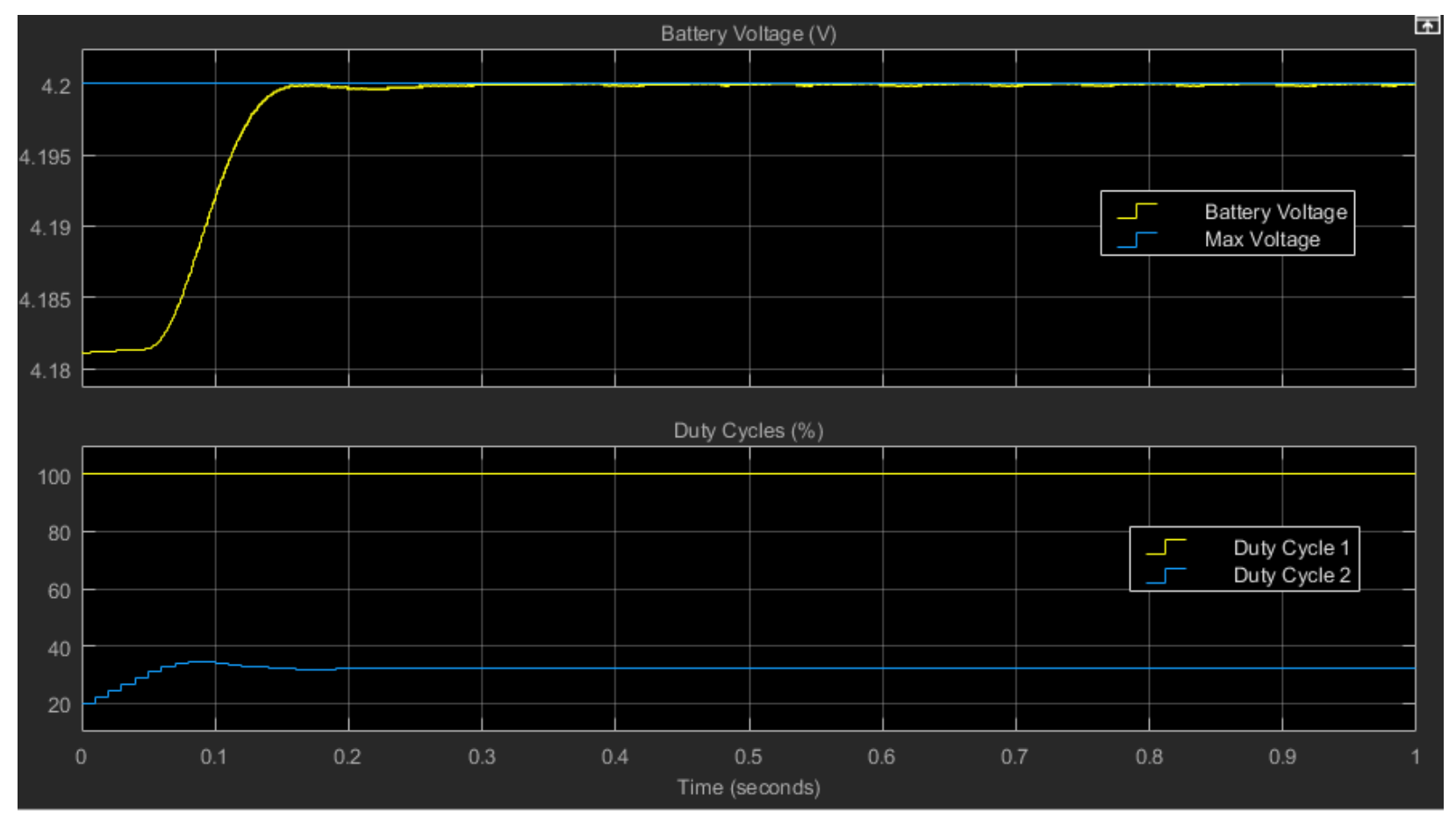

Figure 70. Case: $c=5, d=-4.5$

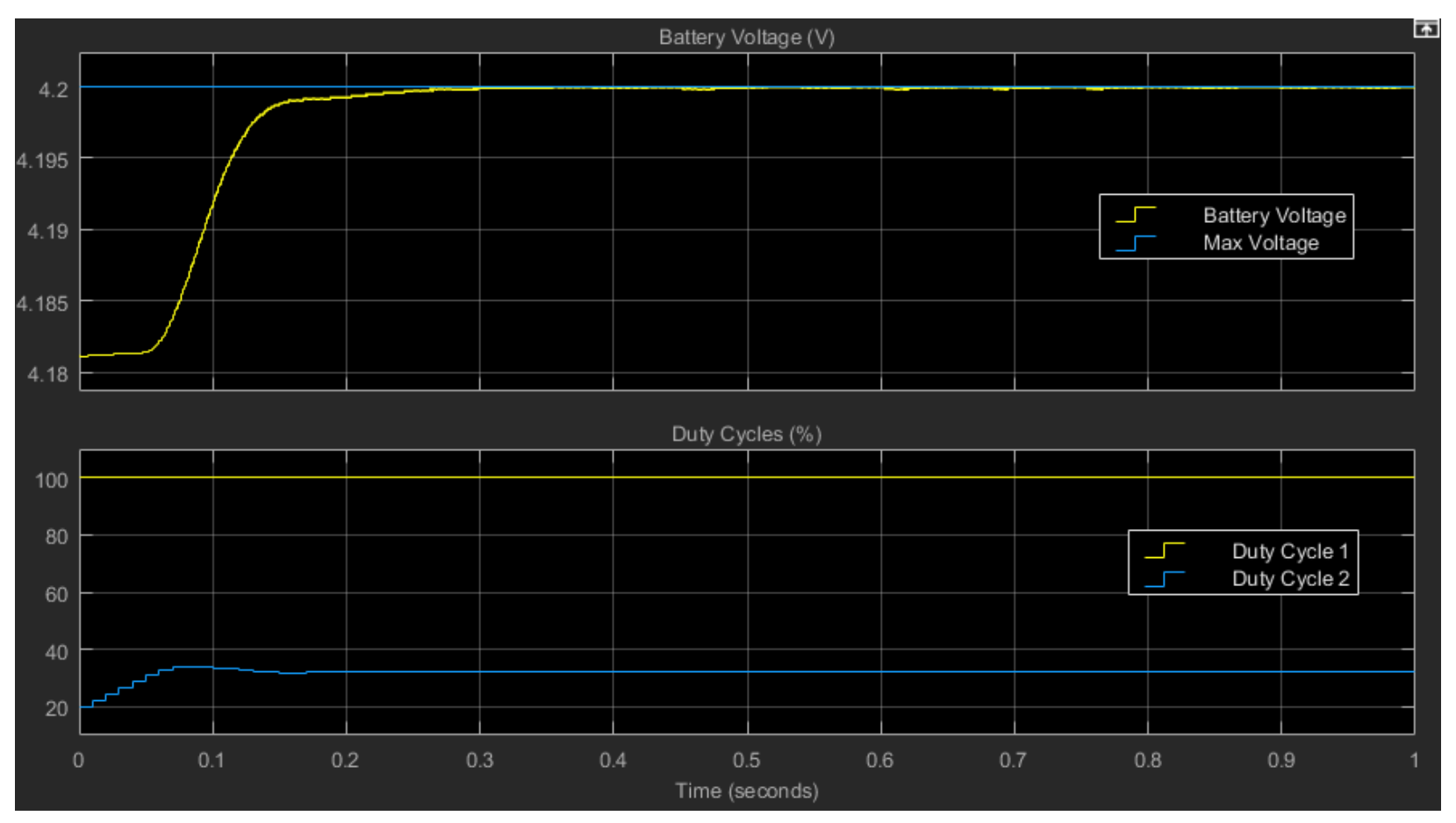

Figure 71. Case: $c=5, d=-5$ 


\section{B.3. Battery Current Control Test Cases}

Parameters $e$ and $f$ of equation 3 are investigated in this section.

$$
\Delta D_{I}=e \frac{\left(I_{\max }-I_{B}\right)}{I_{\max }}+f\left(\Delta I_{B}\right)
$$

\section{B.3.1. Parameter $e$ Test Cases}

For all test cases in this section, parameter $f$ is set to zero.

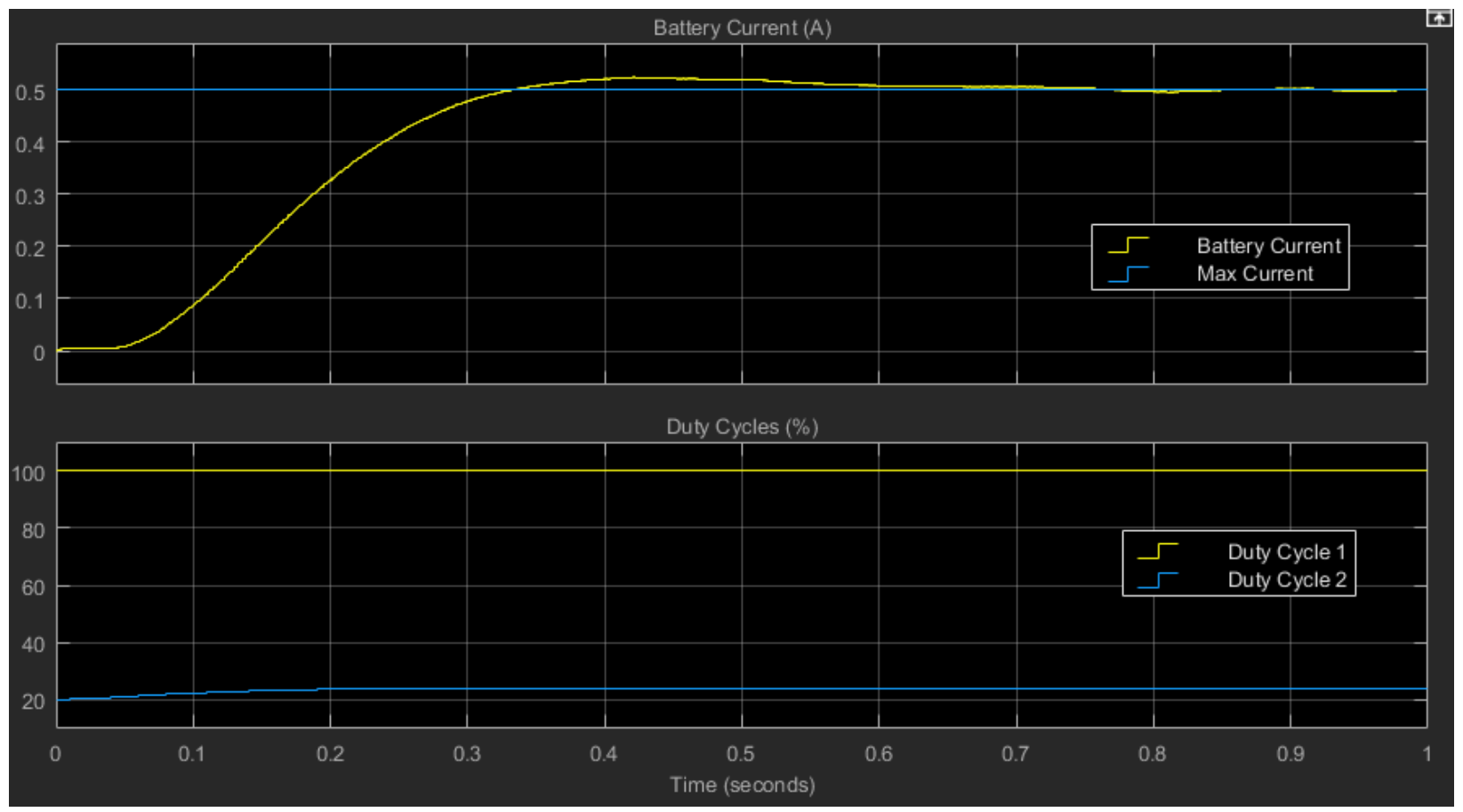

Figure 72. Case: $e=0.0025, f=0$ 


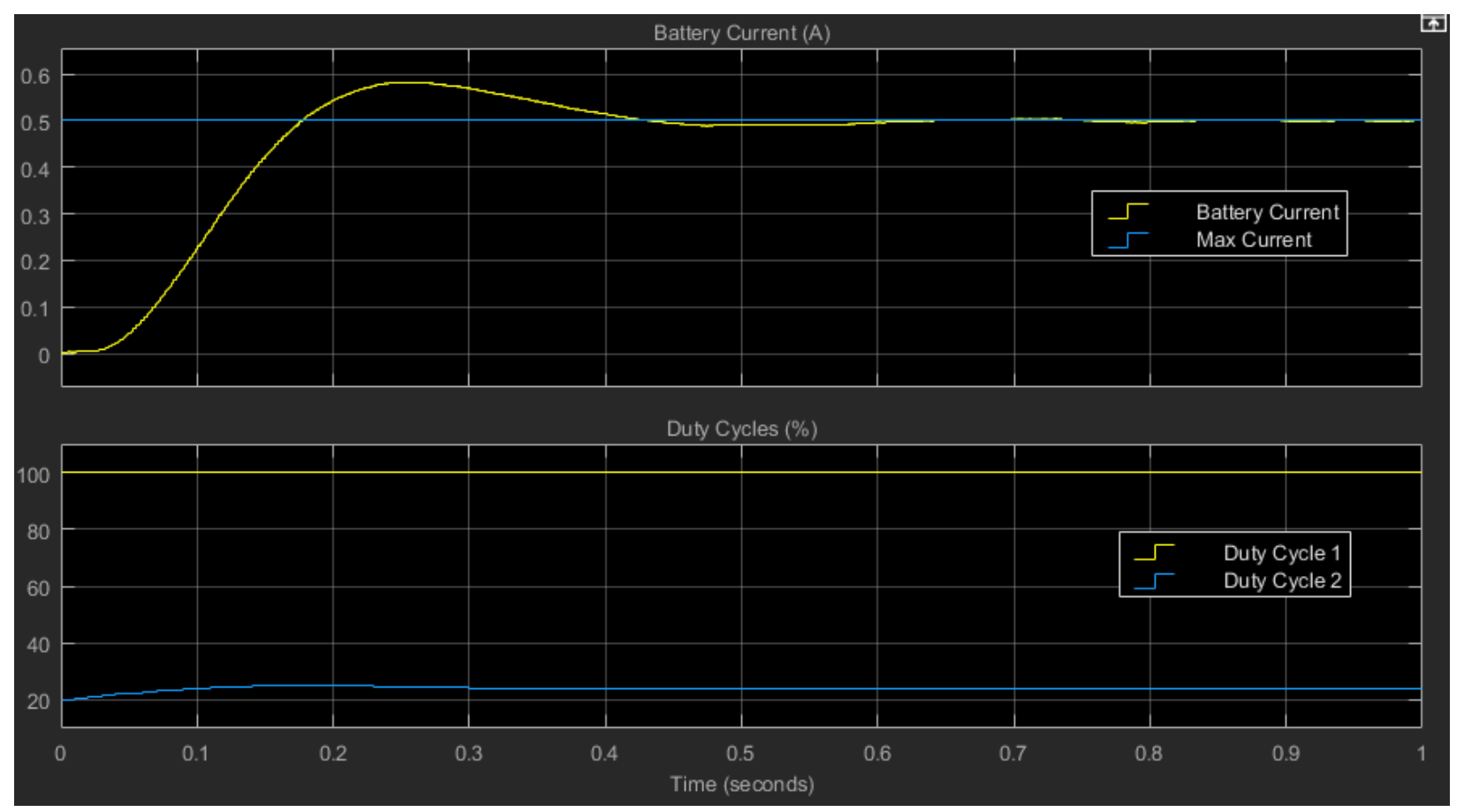

Figure 73. Case: $e=0.005, f=0$

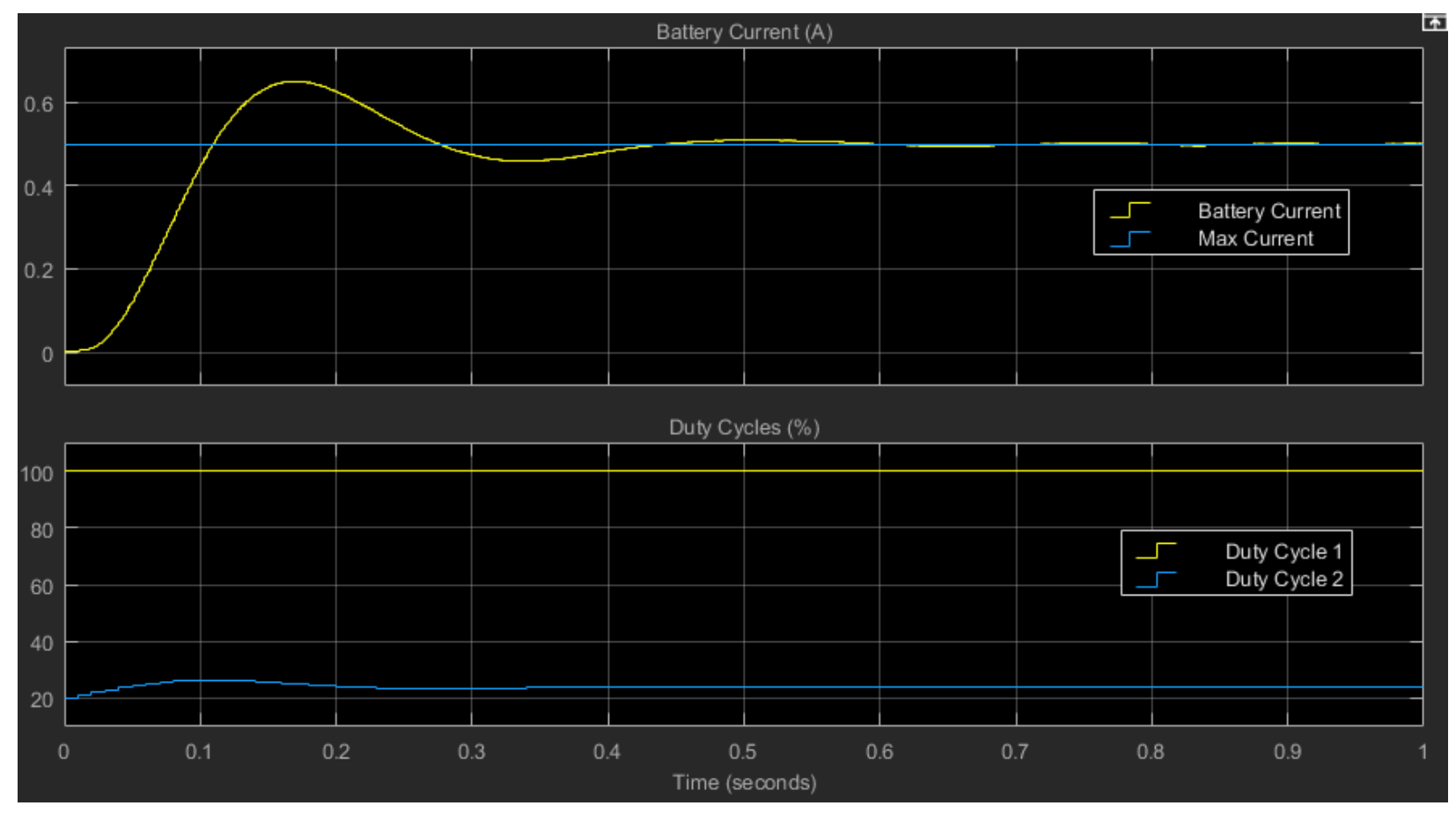

Figure 74. Case: $e=0.01, f=0$ 


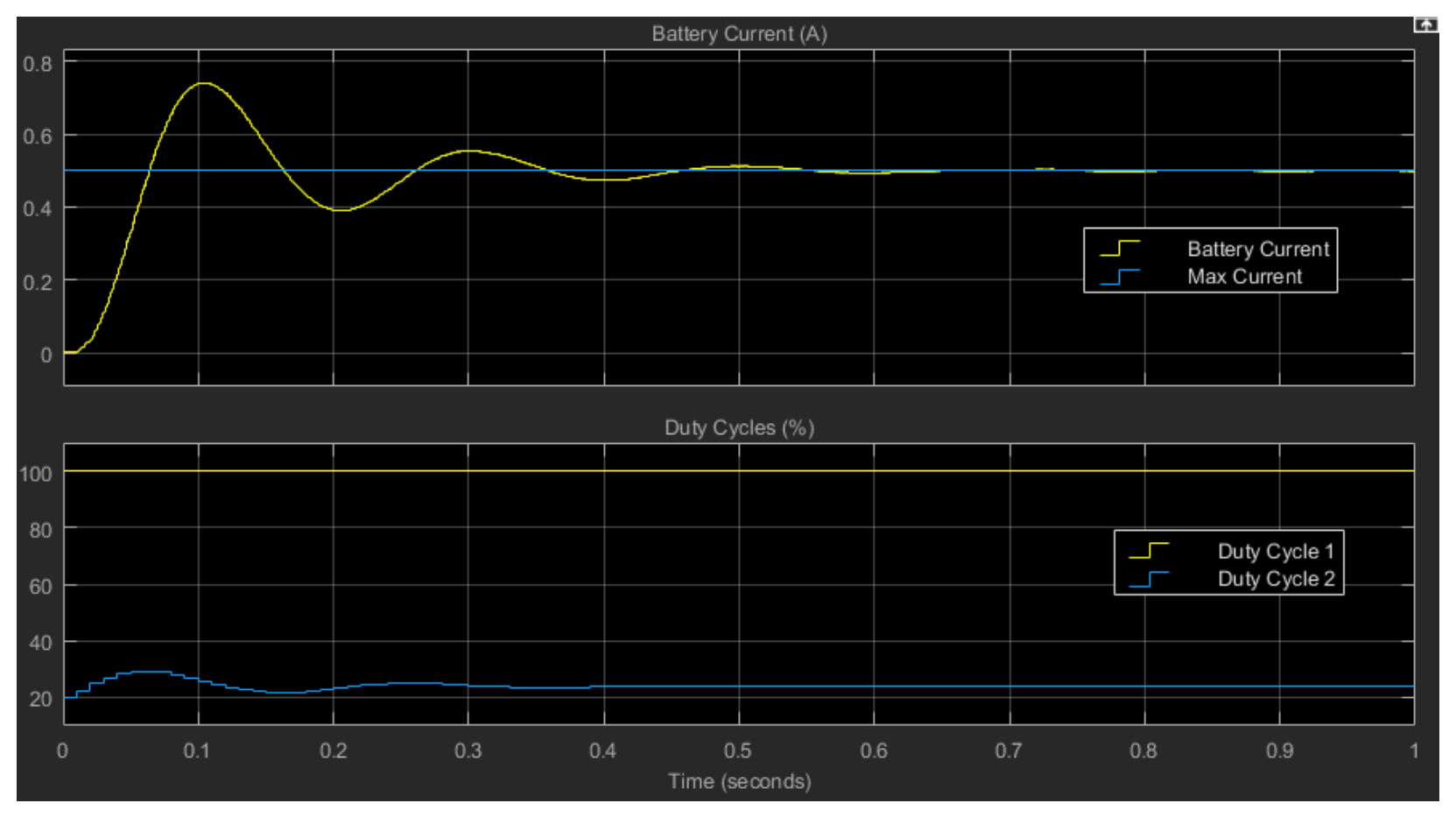

Figure 75. Case: $e=0.025, f=0$

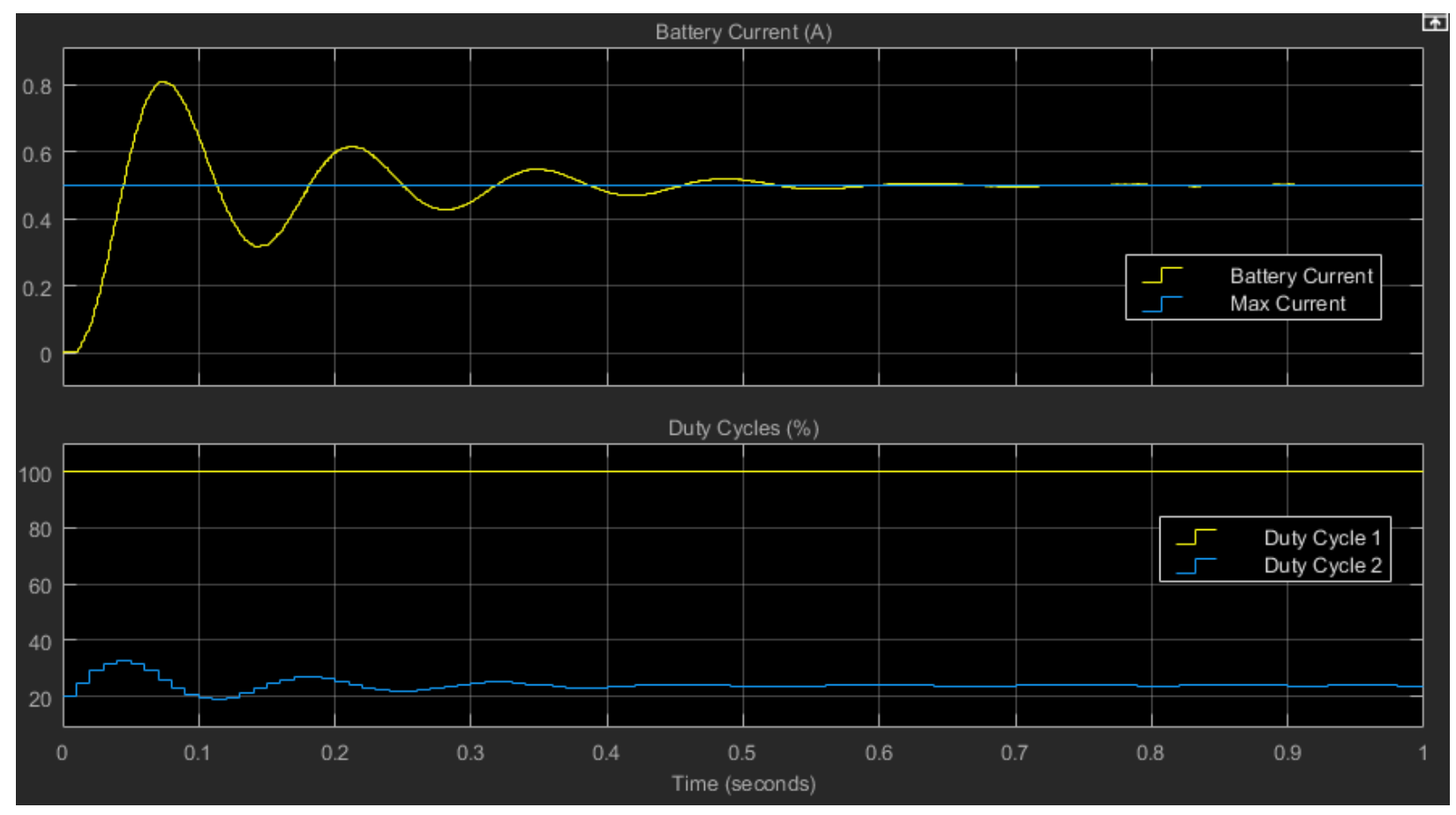

Figure 76. Case: $e=0.05, f=0$ 


\section{B.3.2. Parameter $e$ and $f$ Test Cases}

For all test cases in this section, parameter $e$ and $f$ aren't zero.

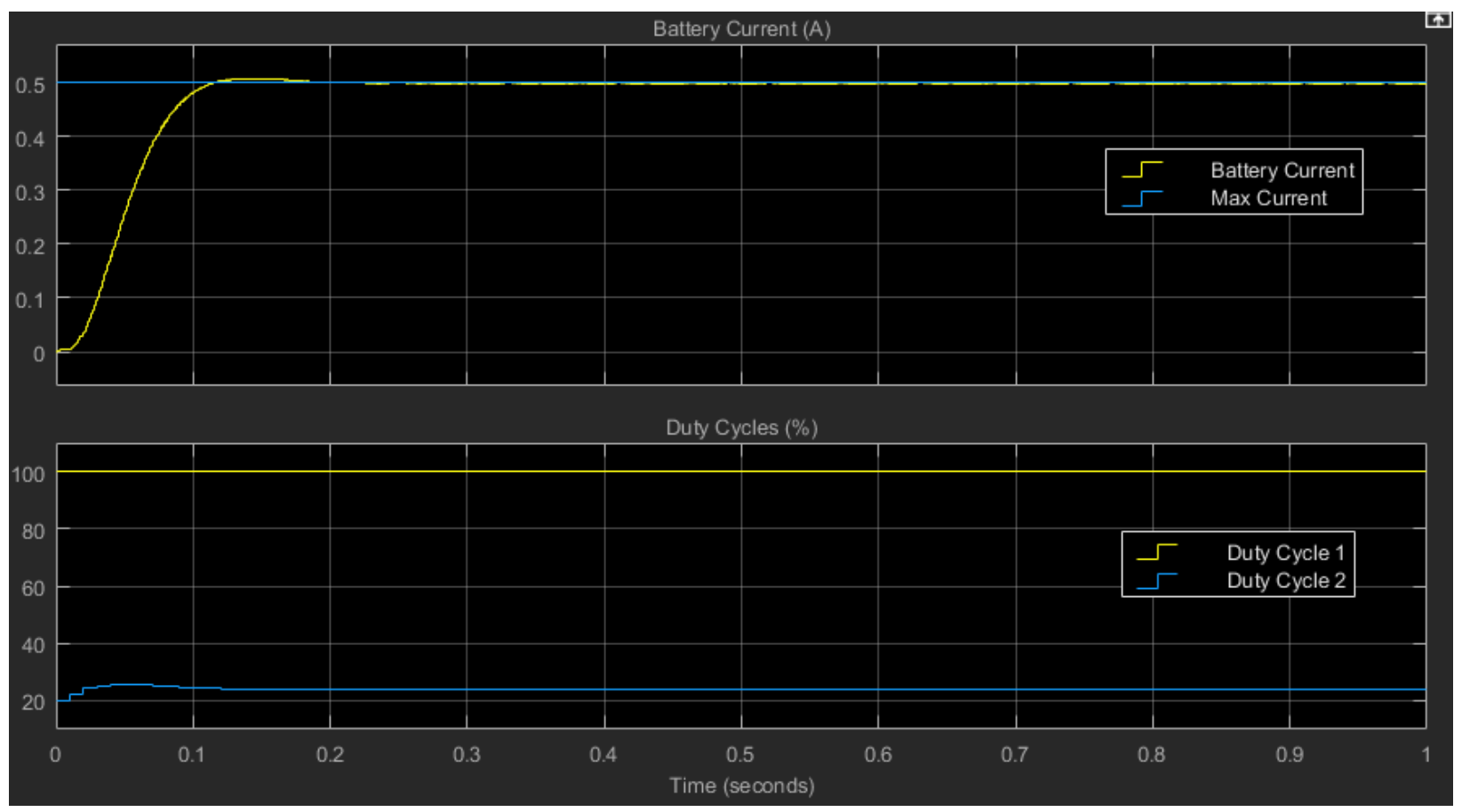

Figure 77. Case: $e=0.025, f=-0.15$ 


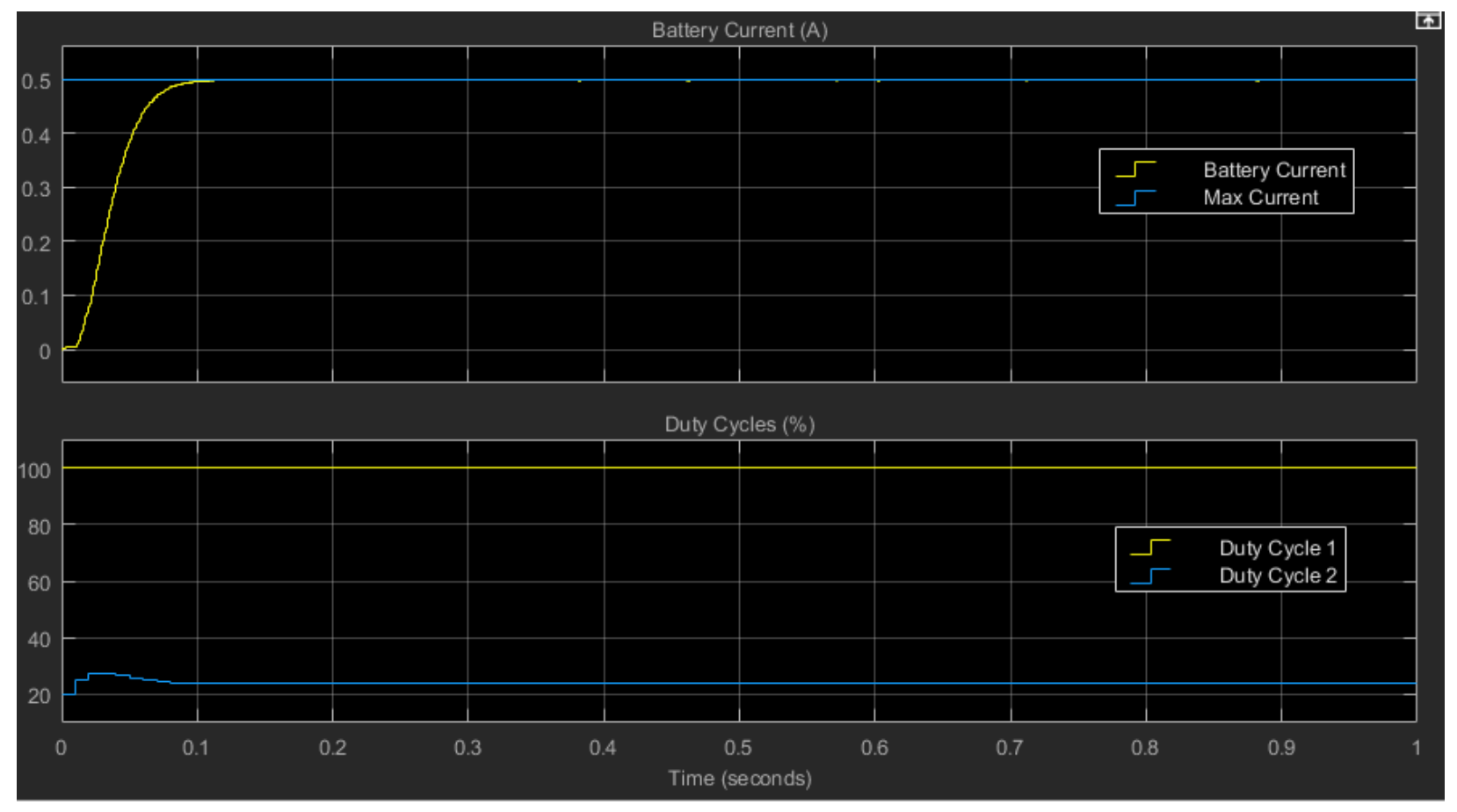

Figure 78. Case: $e=0.05, f=-0.25$

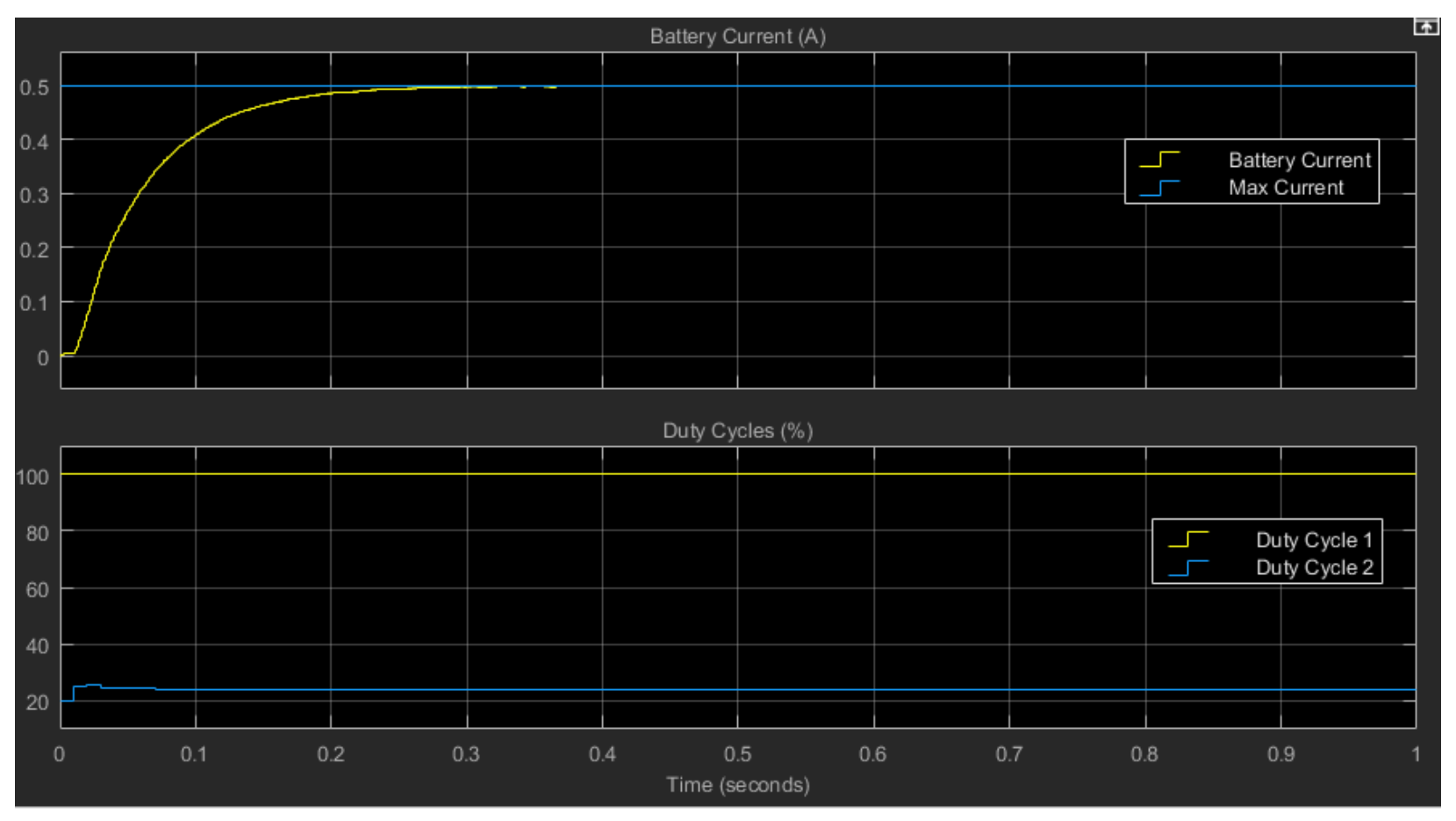

Figure 79. Case: $e=0.05, f=-0.5$ 


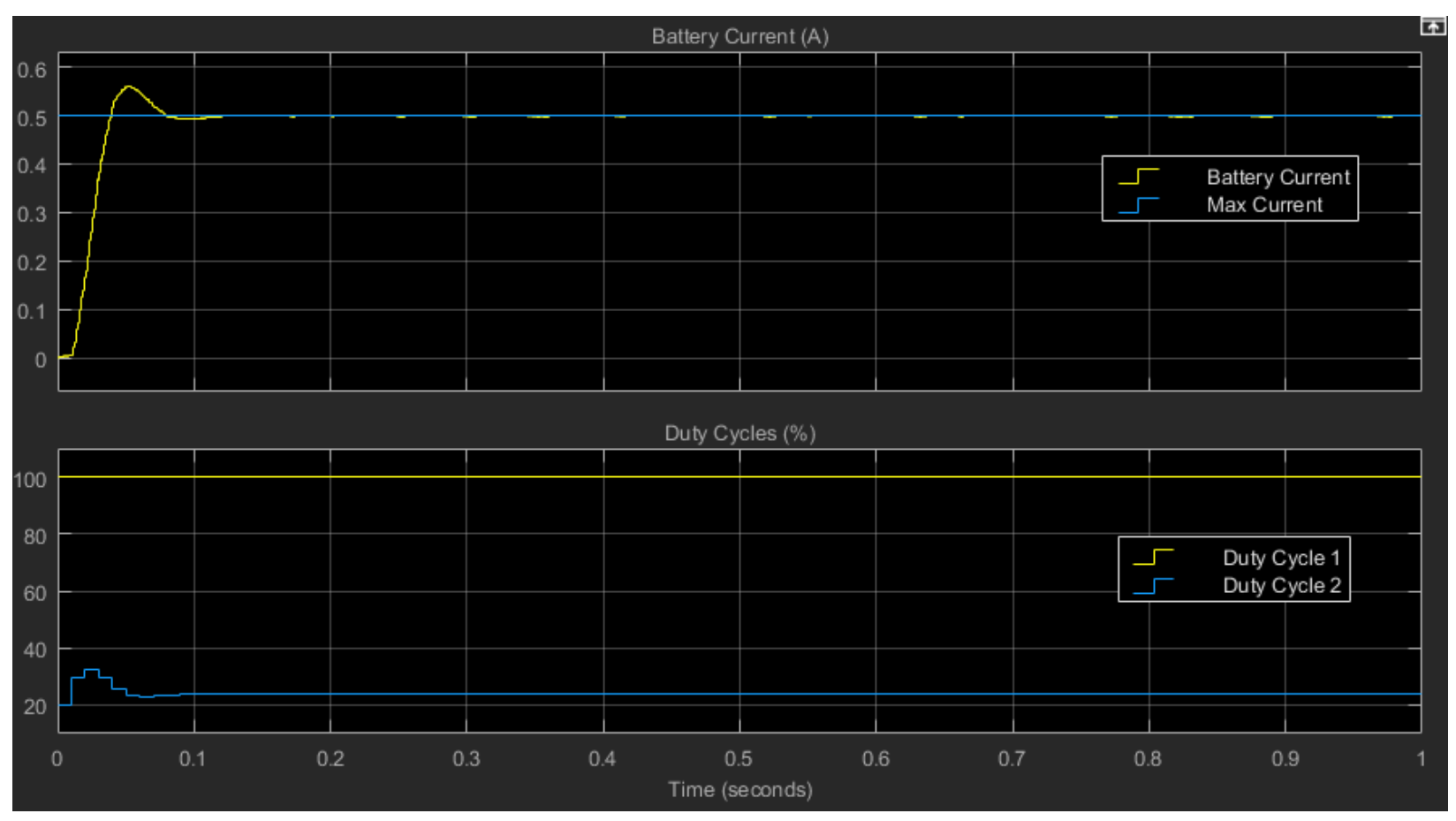

Figure 80. Case: $e=0.1, f=-0.25$

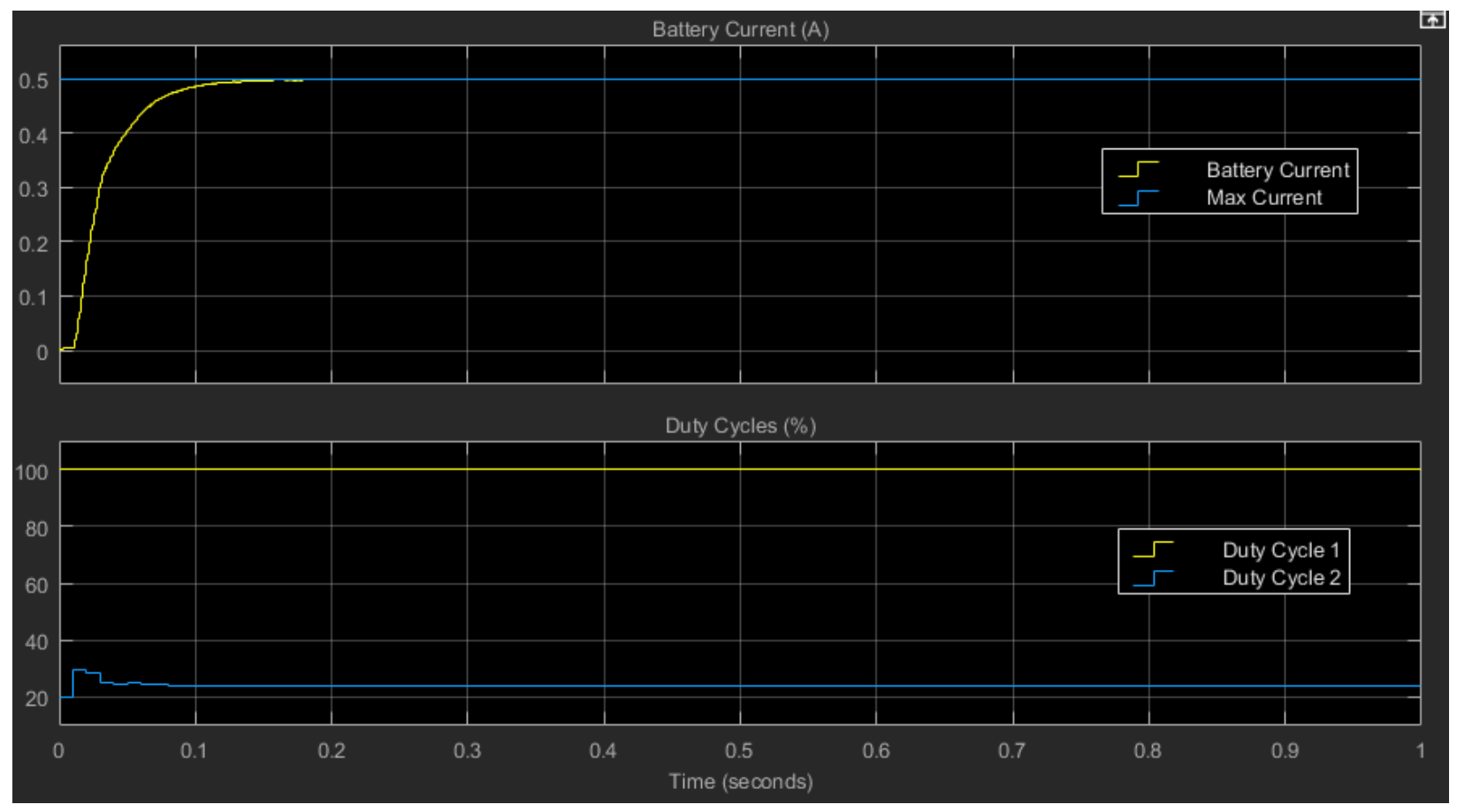

Figure 81. Case: $e=0.1, f=-0.5$ 